\title{
The role of the E3 ubiquitin ligase FBX07-SCF in early-onset Parkinson's disease
}

\author{
Ph.D. Thesis \\ in partial fulfilment of the requirements for the degree \\ "Doctor rerum naturalium" \\ in the Neuroscience Program \\ at the Georg-August-Universität Göttingen, \\ Faculty of Biology \\ submitted by \\ David Brockelt
}

born in

Berlin, Germany

Göttingen 2015

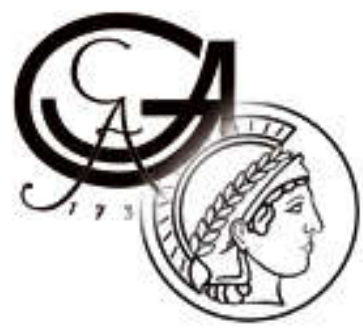




\section{Members of the thesis committee}

Dr. Judith Stegmüller, Reviewer

Department of Cellular and Molecular Neurobiology

Max Planck Institute for Experimental Medicine

Prof. Dr. Tiago Fleming Outeiro, Reviewer

Department of Neurodegeneration and Restorative Research University Medical Center Göttingen

Prof. Dr. Klaus-Armin Nave

Department of Neurogenetics

Max Planck Institute for Experimental Medicine

Date of the oral examination: $19^{\text {th }}$ November, 2015 


\section{Table of contents}

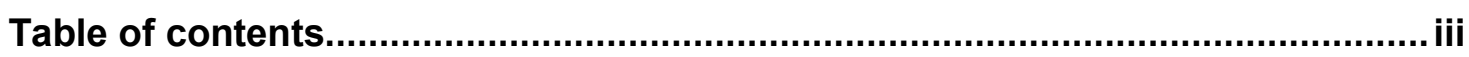

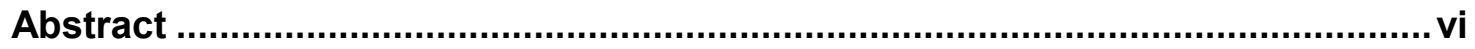

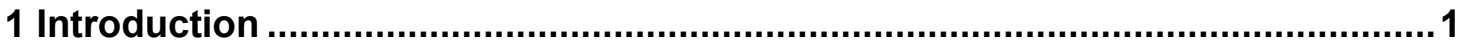

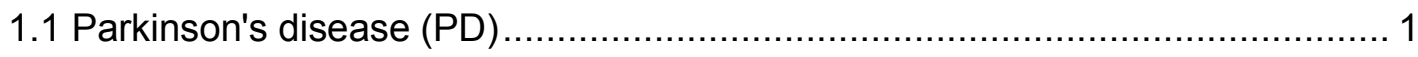

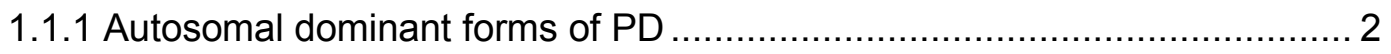

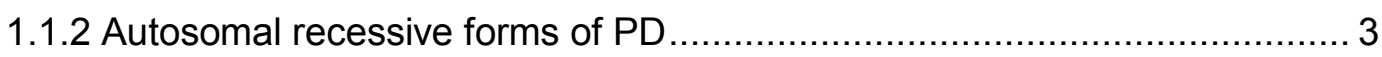

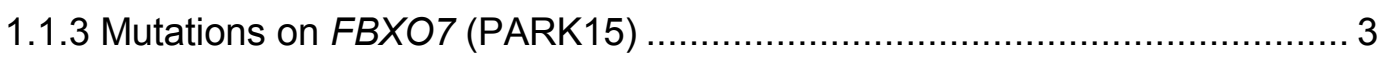

1.2 Parkinson's disease and the ubiquitin-proteasome system (UPS) ................... 5

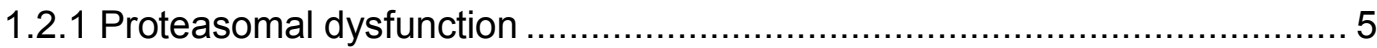

1.2.2 Ubiquitin proteasome system components associated with Parkinson's

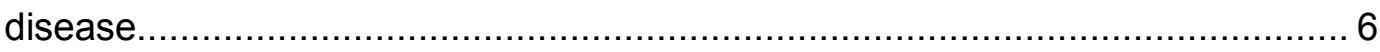

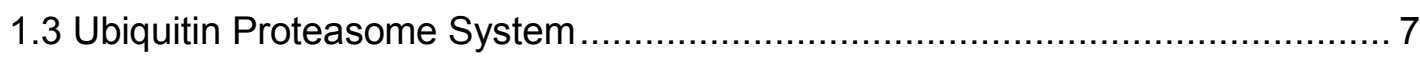

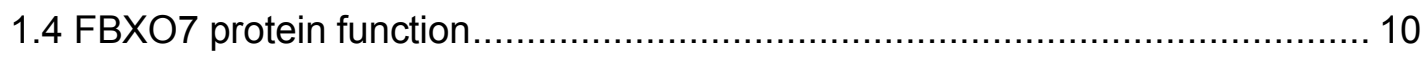

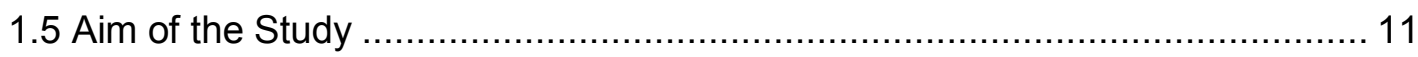

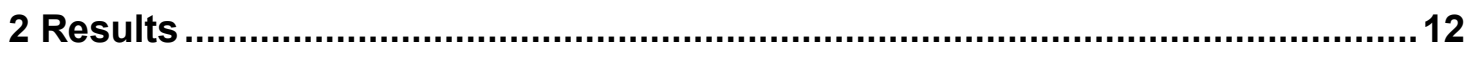

2.1 Generation of a FBXO7 knockout mouse ……….......................................... 12

2.2 FBXO7 is abundantly expressed in the mouse brain and localizes to the

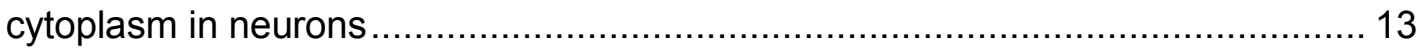

2.3 No $\alpha$-synuclein- or amyloid precursor protein (APP)-positive inclusions in

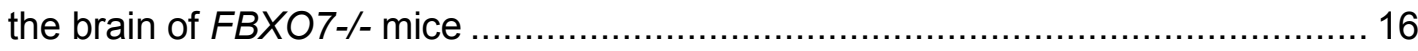

2.4 Increased astrogliosis and elevated levels of apoptosis in the brain of

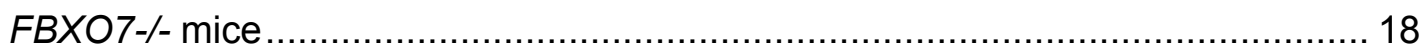

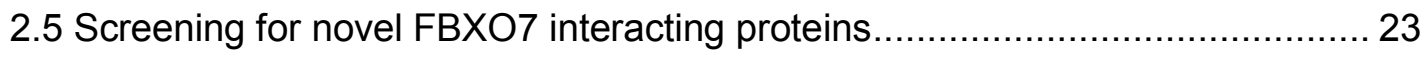


2.6 FBXO7 interacts with members of the MAP1 (microtubule-associated protein) familiy

2.7 The MAP1 family proteins are potential ubiquitination substrates of FBXO7-

SCF

2.8 FBXO7 binds to the proteasome via its novel interaction partner PSMA2 (proteasomal subunit alpha 2).

2.9 FBXO7 ubiquitinates PSMA2 mainly by K63-linked chains with a nonproteolytic outcome.

2.10 FBXO7 is required for proper proteasomal assembly and function. 41

2.10.1 Proteasome activity is reduced as a result of FBXO7-deficiency 41

2.10.2 Loss of FBXO7 does not affect proteasomal gating 42

2.10.3 Loss of FBXO7 causes incomplete assembly of the proteasome holoenzyme.

3 Discussion.

3.1 Systemic loss of FBXO7 results in detrimental effects on the mouse organism.

3.2 FBXO7 and the MAP1 protein family - additional regulators of mitochondrial quality control? 51

3.3 FBXO7 - a novel proteasome regulating protein ................................... 52

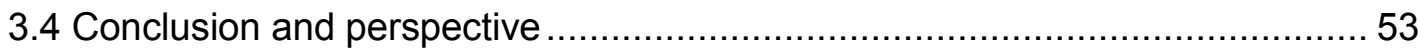

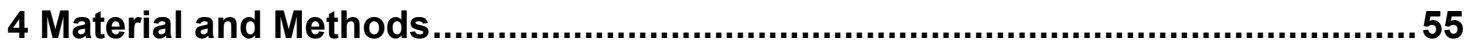

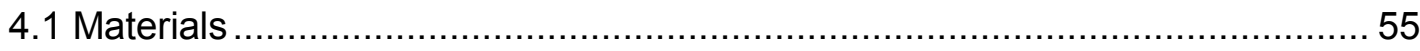

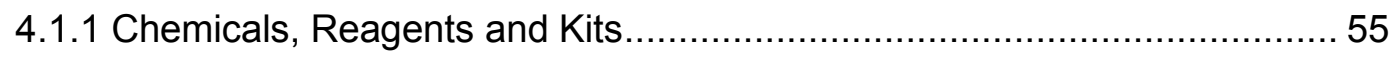

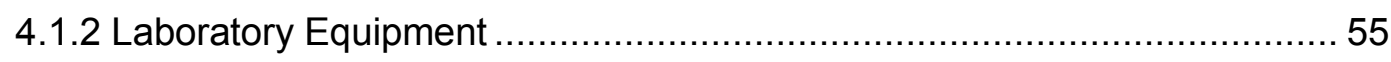

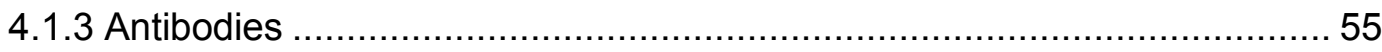

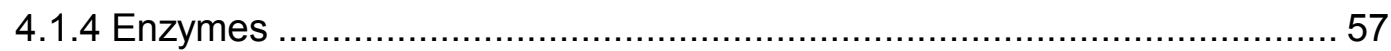

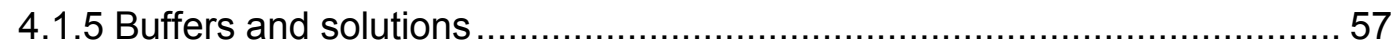

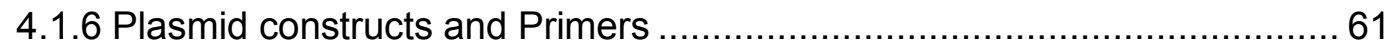




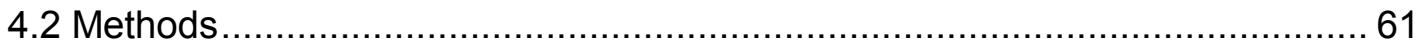

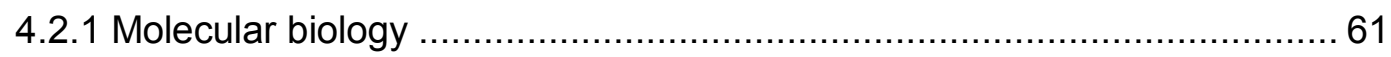

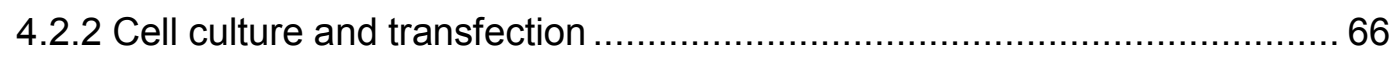

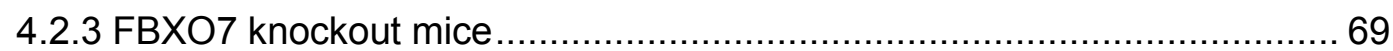

4.2.4 General biochemical methods ...................................................... 70

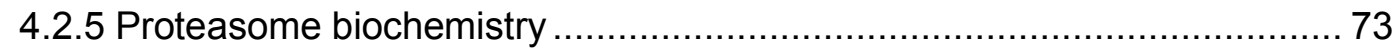

4.2.6 Histological analysis of mouse brain tissue................................. 77

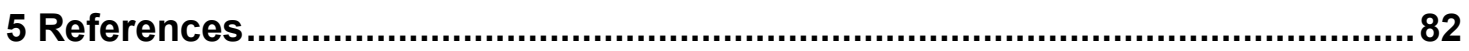

Acknowledgements......................................................................................... v

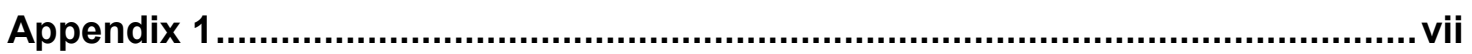

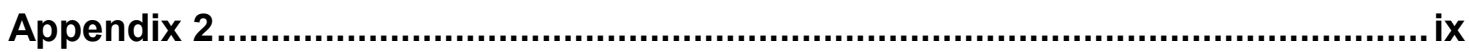

List of abbreviations ................................................................................

Affidavit...............................................................................................

Curriculum Vitae ................................................................................. Xvii 


\section{Abstract}

Parkinson's disease (PD) is a debilitating movement disorder. Growing evidence associates genes with familial forms of the disease. Recently, gene mutations in the FBXO7 (PARK15) gene have been identified in patients with early-onset parkinsonism symptoms and pyramidal tract signs. PARK15 encodes for the E3 ubiquitin ligase FBXO7, whose function in the brain remains to be elucidated. In this study, I report that systemic loss of FBXO7 in mice results in an early-onset motor phenotype and premature death, reminiscent of the PARK15 patients. In neuropatholgical analyses, I find a regional increase in cell death and widespread astrogliosis. At the molecular level, I demonstrate the binding of FBXO7 to the proteasomal core subunit PSMA2 and identify PSMA as non-proteolytic ubiquitination substrate. Interestingly, I show that loss of FBXO7 in the brain leads to reduced proteasome activity as a consequence of defective proteasome integrity. Taken together, I established an FBXO7dependent mechanism of proteasome regulation in neurons, which provides novel insight into the role of the UPS in PD. 


\section{Introduction}

\subsection{Parkinson's disease (PD)}

Parkinson's disease (PD) is the second most common neurodegenerative disorder, which in its sporadic form affects approximately $1 \%$ of the population over the age of 60 years. Clinically, it is characterized by motor impairment including resting tremor, bradykinesia, muscle rigidity and postural instability. Pathological hallmarks comprise the degeneration of the nigrostriatal dopaminergic system, which is thought to be causative for the observed motor symptoms (Sherer et al., 2012). Furthermore, post-mortem brain sections of PD patients show a widespread Lewy body pathology of intracellular inclusions consisting predominantly of the protein alpha-synuclein. The majority of the PD cases appears to be sporadic, but linkage studies and positional cloning have identified mutations causing Mendelian forms in approximately $5-10 \%$ of the cases (Cookson, 2005). Hereditary forms of PD resemble the sporadic form of the disease to a great extent both clinically and pathologically. This suggests that understanding of the associated cellular pathways is not only central to the understanding of the familial cases of PD but could also lead to new mechanistic insights and therefore therapies for sporadic PD. To date, mutations in 18 socalled "PARK" genes or loci have been associated with PD (Table 1.1).

Table 1.1: PD-associated loci and genes.

\begin{tabular}{c|cccc|}
\multicolumn{1}{|c}{} & Gene & Locus & Inheritance & Disease onset \\
\hline PARK1, 4 & SNCA & $4 q 22.1$ & dominant & early onset \\
PARK2 & parkin & $6 q 26$ & recessive & early onset \\
PARK3 & unknown & $2 p 13$ & dominant & late onset \\
PARK5 & UCH-L1 & $4 p 13$ & dominant & late onset \\
PARK6 & PINK1 & $1 p 36.12$ & recessive & early onset \\
PARK7 & DJ1 & $1 p 36.23$ & recessive & early onset \\
\cline { 2 - 5 }
\end{tabular}




\begin{tabular}{c|cccc|} 
PARK8 & LRRK2 & $12 q 12$ & dominant & late onset \\
PARK9 & ATP13A2 & $1 \mathrm{p} 36$ & recessive & early onset \\
PARK10 & unknown & $1 \mathrm{p} 32$ & unclear & late onset \\
PARK11 & GIGYF2 & $2 \mathrm{q} 37.1$ & dominant & late onset \\
PARK12 & unknown & Xq21-q25 & X-linked & late onset \\
PARK13 & HtrA2 & $2 \mathrm{p} 13.1$ & unclear & late onset \\
PARK14 & PLA2G6 & $22 q 31.1$ & recessive & early onset \\
PARK15 & FBXO7 & $22 q 12.3$ & recessive & early onset \\
PARK16 & unknown & $1 \mathrm{q} 32$ & unclear & unclear \\
PARK17 & VPS35 & $16 q 12$ & unclear & unclear \\
PARK18 & EIF4G1 & $3 q 27$ & unclear & unclear
\end{tabular}

SNCA, synuclein, alpha (non A4 component of amyloid precursor); UCH-L1, ubiquitin carboxyl-terminal esterase L1 (ubiquitin thiolesterase); PINK1, PTEN induced putative kinase 1; LRRK2, leucine-rich repeat kinase 2; ATP13A2, ATPase type 13A2; GIGYF2, GRB10 interacting GYF protein 2; HtrA2, HtrA serine peptidase 2; PLA2G6, phospholipase A2, group VI (cytosolic, calcium-independent); FBXO7, Fbox protein 7; VPS35, VPS35 retromer complex component; EIF4G1, eukaryotic translation initiation factor 4 gamma, 1.

\subsubsection{Autosomal dominant forms of PD}

Alpha-synuclein (SNCA) is one example of a direct link between sporadic and hereditary forms of PD. It was the first PARK gene locus to be identified and found in a large family displaying parkinsonism with an autosomal-dominant inheritance pattern and Lewy body pathology (Polymeropoulos et al., 1996). Since then, two types of mutations in the SNCA gene have been recognized: three different point mutations (designated as PARK1) (Kruger et al., 1998; Polymeropoulos et al., 1997; Zarranz et al., 2004) and multiplications of the SCNA gene (PARK4) (Chartier-Harlin et al., 2004; Singleton et al., 2003). Although these mutations are rare, they led to the significant discovery that alpha-synuclein is the major fibrillar component of the Lewy bodies (Spillantini et al., 1997), suggesting the implication of altered alpha-synuclein function in both familial and sporadic PD.

Identification of LRRK2 (Leucine-rich repeat kinase 2; PARK8) mutations provided another important link to sporadic PD, which on one hand is due to the close clinical resemblance with regard to a typical late-onset, levodopa-responsive parkinsonism 
(Funayama et al., 2002). On the other hand, PARK8 is not only the most prevalent form of inherited PD but also frequently found in patients of sporadic PD itself (Bardien et al., 2011) and hence investigation of LRRK2 function and etiology is directly relevant for sporadic PD.

\subsubsection{Autosomal recessive forms of PD}

In contrast to dominantly inherited forms of PD, autosomal-recessive forms typically lead to an early onset of the disease. First identified in Japanese families with juvenile onset of parkinsonism (Kitada et al., 1998), mutations in the parkin gene (PARK2) are the most prevalent cause of early-onset PD (age of onset <40-50 years) (Periquet et al., 2003). Parkin patients clinically resemble sporadic PD patients but a number of additional clinical features, such as pyramidal signs, cerebellar features and psychiatric disease, have also been reported (Corti et al., 2011). Pathologically, they are characterized by a significant loss of dopaminergic neurons in the substantia nigra pars compacta usually with the absence of Lewy bodies (Mori et al., 1998).

Growing evidence implicates the autosomal-recessive genes parkin together with PINK1 (PTEN-induced putative kinase 1; PARK6) as well as DJ-1 (PARK7) in mitochondrial regulation (Dodson and Guo, 2007). Owing to the observation that the parkinsonism-inducing neurotoxin 1-methyl-4-phenyl-1,2,3,6-tetrahydropyridine (MPTP) is a selective inhibitor of mitochondrial complex I (Langston et al., 1984; Markey et al., 1984), the involvement of mitochondrial dysfunction has been proposed as one of the main cellular pathogenic mechanisms in the etiology of PD. Implication of several autosomal-recessive genes in mitochondrial quality control has hence confirmed the role of this organelle in PD and moreover uncovered this cellular system as a potential therapeutic target.

\subsubsection{Mutations on FBXO7 (PARK15)}

Autosomal-recessive mutations of FBXO7 (F-box protein 7; PARK15) were first reported in 2008 (Shojaee et al., 2008) and subsequently associated with hereditary parkinsonism in 2009 (Di Fonzo et al., 2009). Since then, mutations in the gene have been identified in several families (Table 1.2). PARK15 patients display a juvenile onset of the disease with a broad spectrum of symptoms. While most of the so far reported cases show a parkinsonian-pyramidal syndrome (PPS), the phenotypes range from only pyramidal involvement (Shojaee et al., 2008) over a mixed contribution as parkinsonian-pyramidal syndrome (PPS) (Di Fonzo et al., 2009) to only parkinsonism (which also includes also a broad spectrum of disorders) (Lohmann et al., 2015). All patients treated with the amino acid 
precursor of dopamine, levodopa, display a rather short-term response, however, with strong dyskinetic and behavioral side-effects. Other symptoms include dystonia, dysphagia, dysarthria, upgaze limitation, incontinence, cortical atrophy and cognitive decline (Di Fonzo et al., 2009; Lohmann et al., 2015). FBXO7 mutations were found to be mainly homozygous missense alterations with only two exceptions, one heterozygous missense (Lin et al., 2013) and one compound heterozygous mutation paired with a splice site variation (Di Fonzo et al., 2009). While the clinical phenotypes of the identified PARK15 patients are well described, data on underlying pathological mechanisms is still missing.

Table 1.2: Clinical characteristics of PARK15 patients.

\begin{tabular}{|c|c|c|c|c|c|}
\hline Patient code & 10 patients & BO-53 & NIJ-002 & $\begin{array}{l}\text { Family } \mathrm{C} \text {, } \\
\text { patient } 1\end{array}$ & ANK-07 \\
\hline Reference & $\begin{array}{l}\text { (Shojaee et } \\
\text { al., 2008) }\end{array}$ & $\begin{array}{l}\text { (Di Fonzo et } \\
\text { al., 2009) }\end{array}$ & $\begin{array}{l}\text { (Di Fonzo et } \\
\text { al., 2009) }\end{array}$ & $\begin{array}{l}\text { (Paisan-Ruiz et } \\
\text { al., 2010) }\end{array}$ & $\begin{array}{c}\text { (Yalcin- } \\
\text { Cakmakli et al., } \\
2014)\end{array}$ \\
\hline Familiy origin & Iran & Italy & Netherlands & Pakistan & Turkey \\
\hline $\begin{array}{c}\text { Age of onset, } \\
y\end{array}$ & $30 \mathrm{~s}$ & 10 & 18 & 17 & 14 \\
\hline Mutation & R378G & R498X & $\begin{array}{c}1144+1 G> \\
\text { T22M }\end{array}$ & R498X & R498X \\
\hline $\begin{array}{l}\text { Parkinsonian } \\
\text { signs }\end{array}$ & $\begin{array}{c}\mathrm{B}, \mathrm{R} \\
\text { (3 patients) }\end{array}$ & $\mathrm{B}, \mathrm{R}, \mathrm{RT}, \mathrm{PI}$ & $\mathrm{B}, \mathrm{R}, \mathrm{P}$ & $B, R, P$ & $\mathrm{~B}, \mathrm{R}, \mathrm{RT}, \mathrm{PI}$ \\
\hline $\begin{array}{l}\text { Pyramidal } \\
\text { signs }\end{array}$ & $\begin{array}{c}++(\text { all } \\
\text { patients) }\end{array}$ & + & + & + & - \\
\hline $\begin{array}{l}\text { Levodopa } \\
\text { response }\end{array}$ & $\begin{array}{c}+(1 \text { patient } \\
\text { treated })\end{array}$ & + & + & + & + \\
\hline $\begin{array}{l}\text { Levodopa } \\
\text { side effects }\end{array}$ & n.a. & $\begin{array}{l}\text { dyskinesia, } \\
\text { behavioral } \\
\text { disturbances }\end{array}$ & $\begin{array}{l}\text { dyskinesia, } \\
\text { behavioral } \\
\text { disturbances }\end{array}$ & $\begin{array}{c}\text { dyskinesia, } \\
\text { behavioral } \\
\text { disturbances }\end{array}$ & $\begin{array}{c}\text { dyskinesia, } \\
\text { behavioral } \\
\text { disturbances }\end{array}$ \\
\hline
\end{tabular}

$\mathrm{B}$, bradykinesia; R, rigidity; RT, resting tremor; PI, postural instability 


\subsection{Parkinson's disease and the ubiquitin-proteasome system (UPS)}

$F B X O 7$ encodes a protein that functions as an E3 ubiquitin ligase and is hence part of the cell's main protein degradation pathway, the ubiquitin-proteasome system (UPS). Different lines of research have implicated dysfunction of the UPS or its components in the etiology of PD. First hints for this link derived from the observation of inclusion bodies that are ubiquitin-positive. Further research linked several components of the UPS machinery itself to PD.

\subsubsection{Proteasomal dysfunction}

Lewy bodies are protein inclusions and a major pathological hallmark of sporadic PD. The presence of ubiquitin, among other proteins, in the inclusion bodies suggested an involvement of dysfunctional proteasomal clearance mechanisms (Lowe et al., 1988). Indeed, postmortem tissue analyses of PD patients showed a reduction of proteasome activity in the substantia nigra (Furukawa et al., 2002; McNaught et al., 2003), but an increase of activity in other brain regions, such as the cerebral cortex and the striatum (Furukawa et al., 2002), possibly as a compensatory mechanism. The association of reduced proteasome function and pathogenesis of PD was further supported by in vitro studies demonstrating that proteasome inhibition led to degeneration of primary dopaminergic neurons with alphasynuclein and ubiquitin-positive inclusion body formation (Rideout et al., 2005). Moreover, systemic administration as well as nigral stereotactic injection of proteasome inhibitors in rats was reported to induce a behavioral and pathological phenotype resembling PD (McNaught et al., 2002a). However, these findings remain controversial due to a lack of reproducibility (Bove et al., 2006; Hawlitschka et al., 2007; Kordower et al., 2006), and hence in vivo administration of proteasome inhibitors is not considered to be a valid approach to model PD. A more recent study used a genetic approach to delete a subunit of the $26 \mathrm{~S}$ proteasome in a brain region-specific manner. The mouse model showed Lewy body-like inclusions and neurodegeneration in the targeted nigrostriatal pathway or forebrain, demonstrating that proteasome dysfunction is a factor that can directly trigger the pathogenesis of neurodegenerative disease involving protein aggregates and neuronal death (Bedford et al., 2008).

The central position of the proteasome in the cell's functional network and its sensitivity to internal and external stimuli (Demasi et al., 2003; Wang et al., 2010), however, make it difficult to distinguish dysfunctions of the proteasome as a cause or a consequence. This, e.g., holds true for the alpha-synuclein-containing aggregates. While the proteinaceous 
inclusions are ubiquitin-positive and point to deficient proteasomal degradation, it was also reported that wild type or mutant alpha-synuclein as well as alpha-synuclein aggregates in turn impair proteasome activity (Bodner et al., 2006; Fujita et al., 2007; Sun et al., 2005). Further research is hence required to shed light on the mechanisms of proteasome function and regulation in the early phases of disease pathogenesis.

\subsubsection{Ubiquitin proteasome system components associated with Parkinson's disease}

Genetic evidence underscoring the role of UPS components in PD is supported by at least two genes which are part of the pathway of ubiquitin-dependent degradation of proteins and are associated with familial cases of the disease.

Parkin (PARK2) encodes an E3 ubiquitin ligase that attaches ubiquitin to a multitude of substrate proteins. Upon mitochondrial stress, parkin translocates to the outer mitochondrial membrane (Narendra et al., 2008) where it ubiquitinates several substrate proteins, ultimately leading to the removal of defective mitochondria (Tanaka et al., 2010). Some of the mitochondrial ubiquitination targets of parkin are degraded by the proteasome, such as the mitofusins (Chan et al., 2011) which are mitochondrial fusion regulators. Furthermore, parkin is viewed as a protective protein as overexpression can rescue cells from stress-induced unfolded protein response (Imai et al., 2000) or protect against proteasomal dysfunction and toxicity induced by alpha-synuclein (Petrucelli et al., 2002; Yang et al., 2003). This suggests that loss of functional parkin and its E3 ligase activity can lead to pathological alterations.

Another UPS-component that was linked to genetic PD is ubiquitin carboxy-terminal hydrolase 1 (UCH-L1, PARK5) encoding a deubiquitinating enzyme. UCH-L1 is a highly abundant enzyme making up 1-2 \% of the brain protein content (Solano et al., 2000; Wilkinson et al., 1992; Wilkinson et al., 1989). Mutations in UCH-L1 were reported to lead to an in vitro reduction of its deubiquitinase activity (Nishikawa et al., 2003) and shown to result in reduced intracellular ubiquitin levels (Osaka et al., 2003) which could potentially lead to a flawed clearance of proteins. Interestingly, inhibition of UCH-L1 in cell cultures of rat ventral midbrain neurons was shown to cause formation of inclusions and dopaminergic neuron degeneration (McNaught et al., 2002b). Taken together, dysfunction of the proteasome and components of the ubiquitin-attachment pathway have implicated the UPS as another integral cell system in the etiology of PD. 


\subsection{Ubiquitin Proteasome System}

The ubiquitin proteasome system (UPS) is one of the cell's major protein degradation pathways of the cell. It is therefore involved in virtually every cellular process requiring the precise spatial and temporal regulation of protein levels. The signal for a protein to be degraded is the posttranslational modification with the highly conserved, $8.5 \mathrm{kDa}$ protein ubiquitin (Ub). Protein ubiquitination is an enzymatic reaction and involves three steps. In the first step, $\mathrm{Ub}$ is activated in an ATP-dependent manner and thereby covalently bound to the E1 ubiquitin-activating enzyme (E1). Next, ubiquitin is transferred to the E2 ubiquitinconjugating enzyme (E2). Finally, the E3 ubiquitin ligase (E3) mediates the transfer of Ub from the E2-Ub conjugate to the substrate protein (Figure 1.1). Ubiquitin attachment typically occurs on a lysine residue of the substrate protein as a single moiety or polymeric Ub chains. Polyubiquitin chains are formed by attachment of another Ub molecule to one of the seven lysine $(\mathrm{K})$ residues of the preceding $\mathrm{Ub}$. K48-linked polyubiquitin chains are the widely accepted signal for degradation of a protein by the proteasome (Hershko and Ciechanover, 1998).

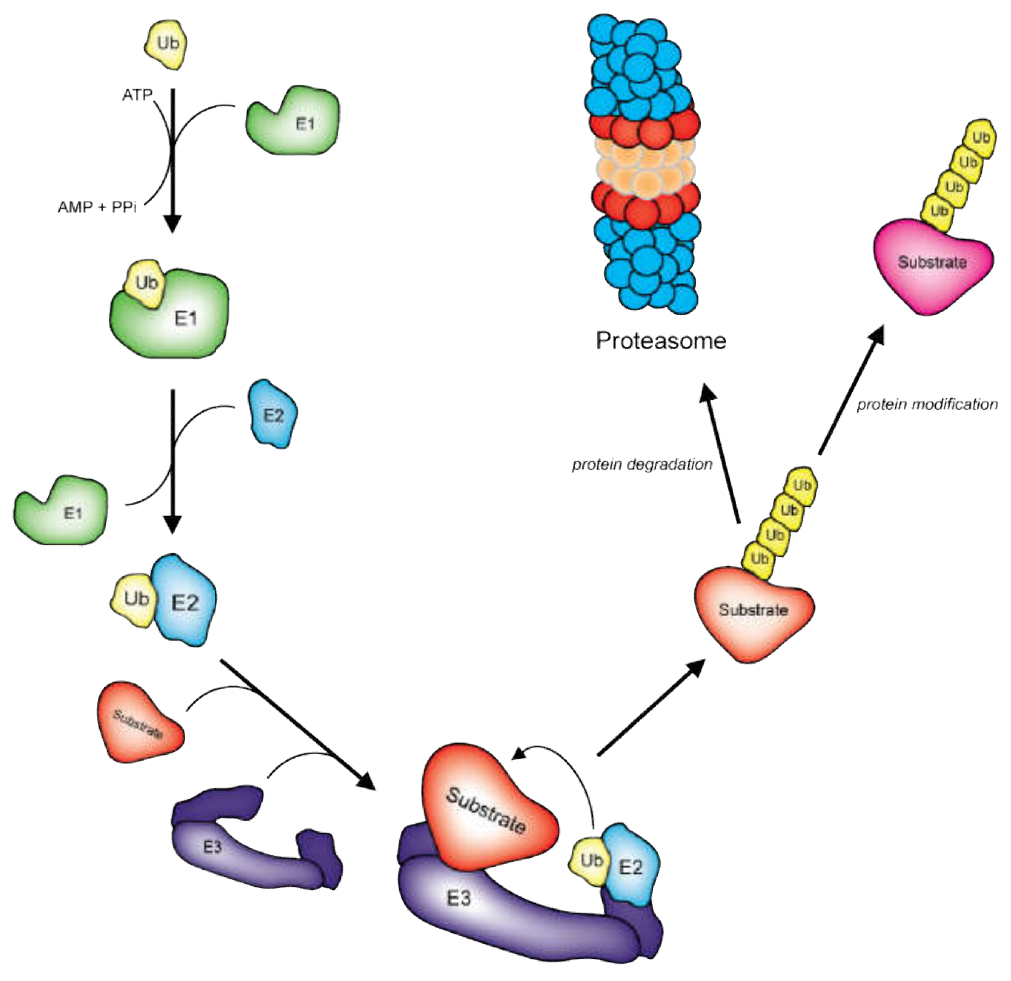

Figure 1.1: The ubiquitin proteasome system (UPS). The E1 enzyme activates ubiquitin (Ub) in an ATPdependent manner and facilitates the transfer to the E2 enzyme. Subsequently, the E3 enzyme binds both, the E2-Ub conjugate as well as the substrate protein to facilitate ubiquitin attachment on the substrate. Ubiquitinated proteins are then either destined for proteasomal degradation or altered in their protein function. 


\subsubsection{The 26S proteasome}

The $26 \mathrm{~S}$ proteasome is a $2.5 \mathrm{MDa}$ macromolecular complex responsible for the ATPdependent degradation of polyubiquitinated proteins. It consists of the proteolytic $20 \mathrm{~S}$ core particle (CP) and one or two 195 regulatory particles (RP) (Figure 1.2). The CP is a hollow barrel-shaped particle comprising of four stacked rings with seven subunits each ( $\alpha 1-7$ / $\beta 1-7$ / $\beta 1-7$ / $\alpha 1-7)$ (Coux et al., 1996). Three of the seven $\beta$-subunits $\left(\beta_{1}, \beta_{2}, \beta_{5}\right)$ harbor proteolytic activity and account for the caspase-, trypsin- and chymotrypsin-like protease activities, respectively (Kisselev et al., 1999; Seemuller et al., 1995). The outer a-rings serve as contact sites for the RP and restrict the access to the proteolytic chamber via their N-terminal tails (Groll et al., 2000). For the targeted degradation of polyubiquitinated proteins, CPs need to be associated with the RP. The RP consists of 19 different subunits and is responsible for recognition of the ubiquitinated substrate, removal of its ubiquitin chain by deubiquitinases and subsequent unfolding and translocation of the substrate polypeptide chain into the core (Tomko and Hochstrasser, 2013).

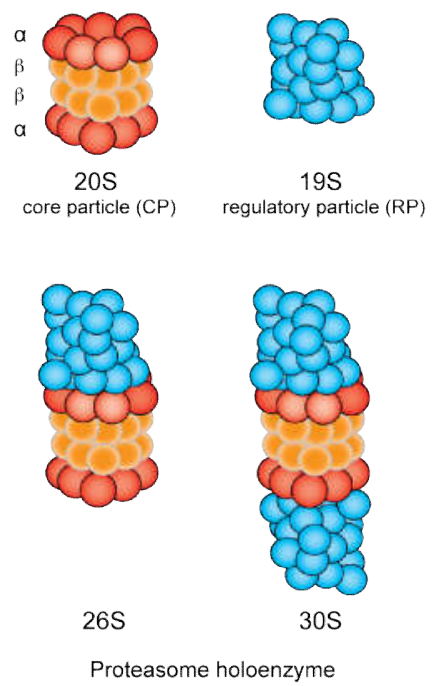

Figure 1.2: Different assembly forms of the proteasome. The $20 \mathrm{~S}$ core particle consists of four heptameric rings of $\alpha$ and $\beta$ subunits. One or two 19S regulatory particles, together with the $20 \mathrm{~S}$ core particle compose the degradation-competent $26 \mathrm{~S}$ or $30 \mathrm{~S}$ proteasome holoenzyme.

\subsubsection{E3 ligases}

In contrast to K48-linked polyubiquitination, which was functionally linked to proteasomal targeting, K63 chaintypes have been mainly associated with modification of protein function. The biological contexts in which the other chaintypes, namely K6, K11, K27, 
K29 and K33, are involved have not yet been investigated in great detail (Ikeda and Dikic, 2008). While examples of E2 enzymes that possess an internal preference for assembly of a specific chaintype have been reported (Chen and Pickart, 1990; Petroski and Deshaies, 2005), a recent study suggests that it is also the E3 ligase itself, which instructs a given E2 to generate a specific chaintype (David et al., 2011). The crucial role of E3 ligases is also reflected in their large number with more than 600 ligases encoded in the human genome. In contrast, only 30 E2 and two E1 enzymes are known to date (Scheffner et al., 1995). Interestingly, one E3 ligase can ubiquitinate more than one substrate and a given substrate in turn, can be targeted by more than one E3 ligase. This further increases the level of complexity with which cellular proteins and hence pathways can be regulated in response to external and internal stimuli.

Based on their mechanism of ubiquitin-attachment, E3 ligases can be classified into two main families: HECT-type and the RING-type E3 ubiquitin ligases. HECT (homologous to E6AP C-terminus) ligases recruit the E2-Ub conjugate and transiently form a thioester with the ubiquitin, prior to catalyzing its attachment to the substrate (Pickart and Eddins, 2004). RING (Really Interesting New Gene) ligases in contrast act as a scaffold that facilitates the ubiquitin transfer from the E2 enzyme to the substrate by bringing both into spatial proximity (Pickart and Eddins, 2004). RING E3 ligases exist as monomers or as multimeric complexes. FBXO7 (PARK15), for example, is part of a multimeric E3 ligase complex, the so-called SCFcomplex.

\subsubsection{SKP1-cullin1-F-box protein (SCF) complex}

SCF-type multisubunit E3 ligases were first identified in yeast (Feldman et al., 1997; Skowyra et al., 1997) and were initially implicated in the cell cycle. The two main functions of an E3 ligase - E2-Ub recruitment and substrate binding - are performed by different subunits of the complex (Figure 1.3). Cullin1 (Cul1) acts as the scaffold protein and binds to the RING-box protein 1 (Rbx1), which recruits the E2-Ub conjugate. The small adaptor protein Sphase kinase-associated protein 1 (SKP1) binds to the F-box protein (FBP) (Cardozo and Pagano, 2004). FBPs are responsible for the critical step of substrate-recognition and recruitment to the complex. While SKP1, Cul1 and Rbx1 represent the ligase core, the FBP is the interchangeable subunit, enabling the SCF E3 ligase to rapidly adapt to the cell's needs by ubiquitinating different subsets of proteins at a time. All 68 members of the mammalian FBP family contain the name-giving F-box domain, an approximately 40 amino acid long stretch, which enables the FBP to form the SCF complex. Based on other structural motifs 
FBPs have been further categorized into FBXLs, FBXWs and FBXOs. FBXLs contain leucinerich repeats while FBXWs contain WD40 domains and FBXOs comprise "other" or no typical structural motifs (Jin et al., 2004).

Despite the large number of identified FBPs, studies on their individual biological functions are sparse. Recent data implicate several FBPs in the context of neurodevelopment and neurodegenerative disease. FBXW8 and FBXO31, e.g., were shown to govern cerebellar development by regulating dendrite and axon growth, respectively (Litterman et al., 2011; Vadhvani et al., 2013). FBXO2 on the other hand was reported to be involved in regulation of the amyloid precursor protein either indirectly by targeting $\beta$-secretase or directly by targeting the amyloid precursor protein itself for degradation (Atkin et al., 2014; Gong et al., 2010). Since the discovery of autosomal-recessive mutations in familial cases of PD, FBXO7 is gaining increasing attention (Shojaee et al., 2008).

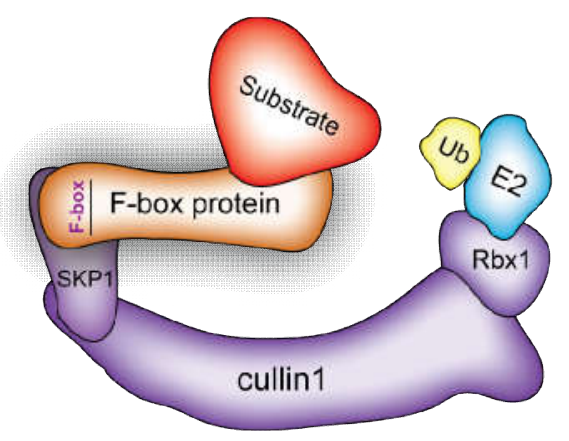

Figure 1.3: The SKP1-cullin1-F-box protein (SCF) complex. The SCF complex is a multimeric E3 ligase, which consists of the core components cullin-1, SKP1 and Rbx1. While Rbx1 recruits the E2-Ub conjugate, SKP1 and cullin-1 facilitate the binding of F-box proteins which in turn recruit the substrate.

\subsection{FBX07 protein function}

The first studies implicated FBXO7 in cell cycle regulation via its reported ubiquitination substrates HURP (hepatoma up-regulated protein) (Hsu et al., 2004) as well as clAP1 (cellular inhibitor of apoptosis 1) and TRAF2 (TNF receptor-associated factor2) (Chang et al., 2006; Kuiken et al., 2012). FBXO7 was furthermore reported to facilitate the cyclin D/cdk6 interaction, leading to an increase in cdk6 activity and suggesting that $F B X O 7$ is a potential oncogene (Laman, 2006). Along the same line of research, FBXO7 was shown to negatively regulate the proliferation and differentiation of hematopoietic progenitor cells (Lomonosov et al., 2011) and that reduced FBXO7 expression in a hypomorphic mouse model led to anemia (Randle et al., 2015). 
While involvement of $\mathrm{FBXO7}$ in cell cycle regulation is interesting, it does not account for its anticipated role in postmitotic neurons given that FBXO7 mutation lead to familial forms of PD, a neurodegenerative disease. Examination of PARK15 patient skin fibroblasts uncovered that two of the so far identified point mutations leads to a loss of protein stability as most of the main FBXO7 protein isoform 1 was depleted (Zhao et al., 2011). Two interesting lines of research imply FBXO7 in regulation of the mitochondrial and proteasomal systems. FBXO7 was shown to interact with PINK1 and Parkin, which are both involved in mitochondrial quality control. Consequently, reduced expression of FBXO7 in SH-SY5Y cells lead to faulty translocation of parkin to mitochondria, a key step in mitophagy (Burchell et al., 2013). The evidence connecting FBXO7 to the proteasomal system has so far been more indirect. FBXO7 harbors a ubiquitin-like (Ubl) domain at its N-terminus, which is commonly found in proteins that regulate proteasomal function (Hartmann-Petersen and Gordon, 2004). Additionally, it was shown to dimerize with the proteasomal inhibitor 31 (PI31) via a globular domain of 150 amino acids that is structurally quite similar and has hence been termed FP (FBXO7-PI31) domain (Kirk et al., 2008). While studies in Drosophila associate nutcracker, an orthologue of FBXO7 with low sequence similarity, with DmPI31 in the control of proteasome activity (Bader et al., 2011), FBXO7's function in the control of mammalian proteasome activity still remains elusive.

\subsection{Aim of the Study}

Mutations in the FBXO7 (PARK15) gene are associated with familial PD. While the patients present with a complex spectrum of motor deficits, their neuropathology remains elusive. Also, it remains unclear how the mutations affect protein function. Owing to a report on loss of FBXO7 expression in skin fibroblasts isolated from PARK15 patients, I took a lossof-function genetics approach. I investigated the systemic loss of FBXO7 in a mouse model and characterized its neuropathological features. In addition, I examined the function of FBXO7 by screening for and establishing novel interaction partners and potential ubiquitination substrates, which provide insight into the neuronal role of the E3 ligase FBXO7SCF. 


\section{Results}

\subsection{Generation of a FBXO7 knockout mouse}

To gain further insights into FBXO7's function in the brain, we generated a FBXO7 conventional knockout mouse using ES cells purchased from the International Knockout Mouse Consortium. Exon 4 of the FBXO7 gene is flanked by FLP-recognition target (FRT) and loxP sites, a lacZ reporter cassette and a neomycin selection cassette (Figure 2.1 a). After successful breeding with the Ella-Cre driver line, exon 4 and the neomycin selection cassette were excised leading to the expression of truncated FBXO7 and $\beta$-Galactosidase ( $\beta$ Gal) proteins driven by the endogenous FBXO7 promoter and terminated by a polyadenylation $(\mathrm{pA})$ signal. Subsequently, successful disruption of the $F B X O 7$ gene was confirmed by a genotyping PCR (Figure 2.1 b, kindly provided by Siv Vingill). I furthermore validated the successful generation of the FBXO7 knockout mouse at the protein level by immunoblotting of different brain tissues with the FBXO7 and $\beta-G a l$ antibodies. As expected, FBXO7+/- mice showed reduced and FBXO7-/- mice a complete loss of FBXO7 protein levels

(Figure $2.1 \mathrm{c}$ ). This result also validated the specificity of the $\mathrm{FBXO7}$ antibody used in this study. Vice versa, $\beta-G a l$ expression was absent from $F B X O 7+/+$ mice and showed half the dose in FBXO7+/- as compared to FBXO7-/- mice (Figure $2.1 \mathrm{~d}$ ).

At postnatal day (P) 18, FBXO7-/- mice displayed reduced body size (Figure 2.1 e). Even though FBXO7-/- mice were born at the expected Mendelian ratios, both genders of knockout mice did not live beyond the third postnatal week (Data Siv Vingill). Basic behavioral testing and general examination of the mice performed by Siv Vingill showed that FBXO7-/mice displayed kyphosis and mild hind limb clasping. Due to their reduced body size, physical weakness and motor deficits, FBXO7-/- mice were not examinable with the rotarod paradigm or other more demanding motor tests. Deletion of one FBXO7 allele, however, did not result in haploinsufficiency since $\mathrm{FBXO7+/-}$ mice displayed normal body weight and motor performance during a 12 months examination period. Altogether, our results suggest that complete loss of $\mathrm{FBXO7}$ in the mouse model system leads to a rapid decline of physical health state as well as early-onset motor symptoms. 


\subsection{Generation of a FBXO7 knockout mouse}

a

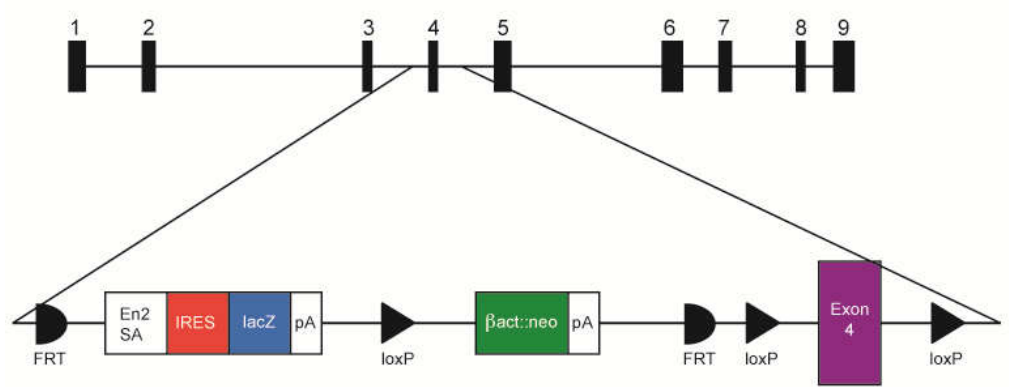

C

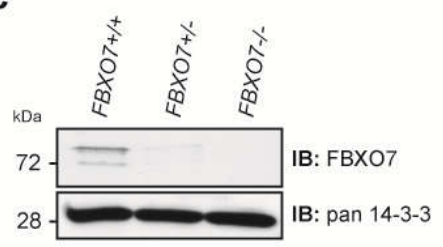

d

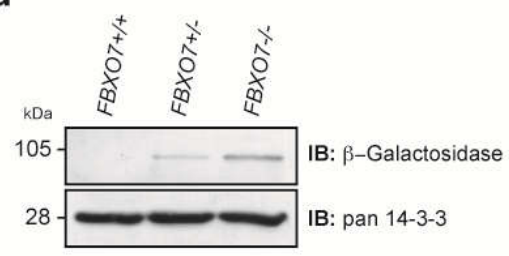

b

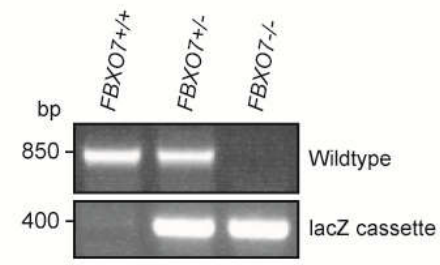

e

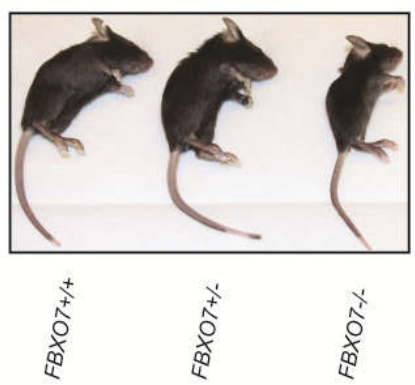

Figure 2.1: Validation of the FBXO7 knockout mouse: (a) Schematic representation of the mutated allele, in which exon 4 of the FBXO7 gene is flanked by loxP sites. The transgenic construct comprises FRT sites, a splice acceptor site (En2-SA), a lacZ reporter cassette as well as a $\beta$-actin:neomycin selection cassette. (b) Representative image of a genotyping PCR of $\mathrm{FBXO7+/+}$, FBXO7+/- and FBXO7-/- mice using primers designed around exon 4 to test for presence or absence of the FBXO7 gene or the $\beta$-Gal reporter cassette, respectively. Image was kindly provided by Siv Vingill. (c) P18 midbrain lysates of $F B X O 7+/+, F B X O 7+/-$ and FBXO7-/- mice were subjected to immunoblotting with the FBXO7 and 14-3-3 antibodies. The latter served as a loading control. (d) P18 cortical lysates of $F B X O 7+/+, F B X O 7+/-$ and FBXO7-/- mice were subjected to immunoblotting with the $\beta-$ Galactosidase and 14-3-3 antibodies. The latter served as a loading control. (e) Picture of $\mathrm{P} 18$ FBXO7+/+, FBXO7+/- and FBXO7-/- mice.

\subsection{FBXO7 is abundantly expressed in the mouse brain and localizes to the cytoplasm in neurons}

In order to investigate the spatial distribution of FBXO7 in the mouse brain, I took advantage of the lacZ expression cassette driven by the endogenous FBXO7 promoter. For this, I subjected sagittal brain sections of P18 FBXO7+/+ and FBXO7-/- mice to lacZ staining. While there was no staining detectable in the $F B X O 7+/+$ brain (Figure 2.2 f), I found abundant $\beta-G a l$ expression in the FBXO7-/- brain (Figure 2.2 a, d) including the cortex, hippocampus, thalamus, midbrain regions and brainstem. In the cortex, the signal was more prominent in the deeper layers, while the hippocampus displayed a strong signal throughout its major regions (Figure 2.2 b, c). A higher magnification of the cerebellar layers revealed $\beta$ Gal expression in the Purkinje cell layer (PCL) and very low expression in the cerebellar 

cytoplasm in neurons

granule neurons of the internal granular layer (IGL) with a few stained cells presumably representing interneurons (Figure 2.2 e). Interestingly, FBXO7 expression was not only restricted to neurons but could be abundantly observed in the white matter tracts of the striatum, the corpus callosum and the white matter region of the cerebellum indicating the expression of FBXO7 in oligodendrocytes in the CNS (Figure $2.2 \mathbf{a}, \mathbf{b}, \mathbf{e}$ ). Collectively, these results establish the expression of $\mathrm{FBXO} 7$ in neuronal and oligodendroglial cells in the mouse brain.

a

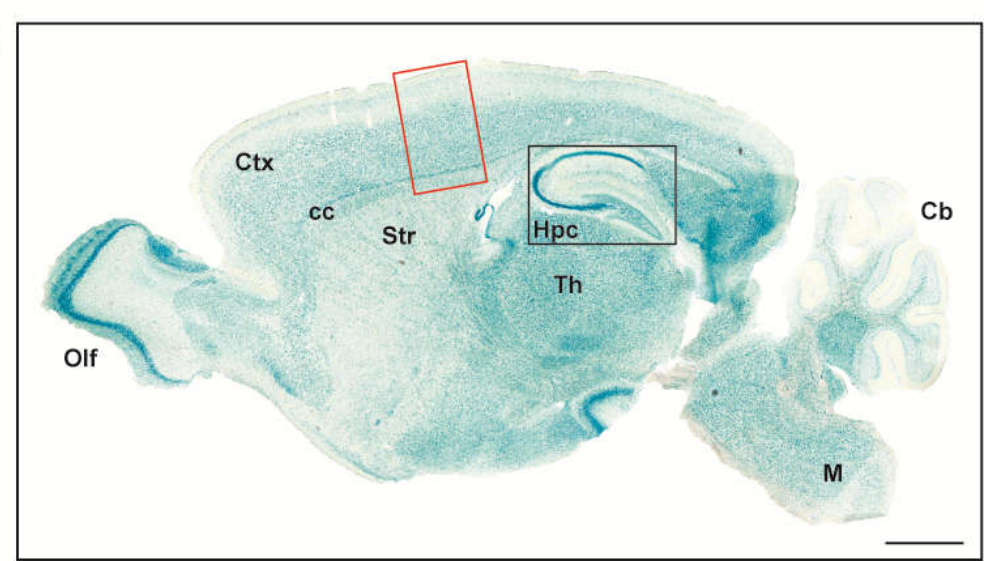

b
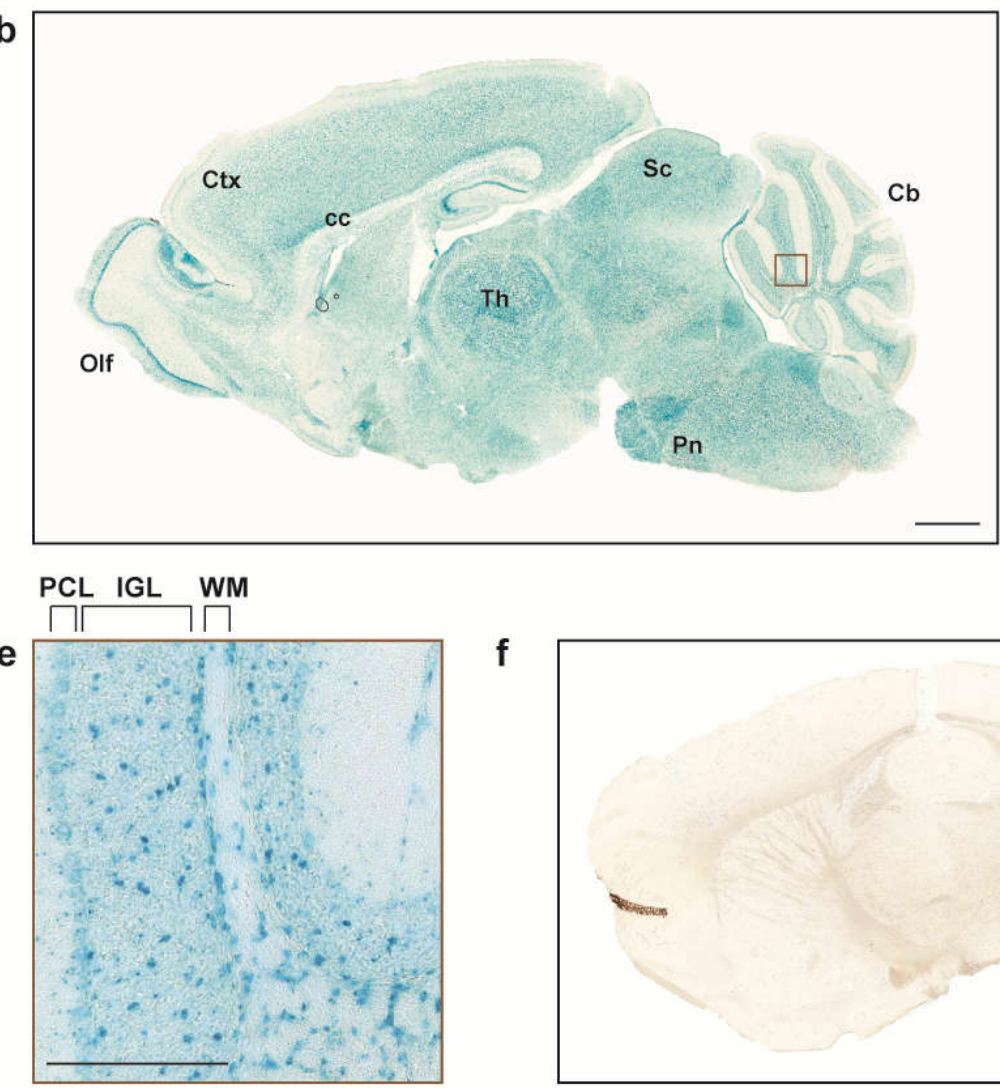

c

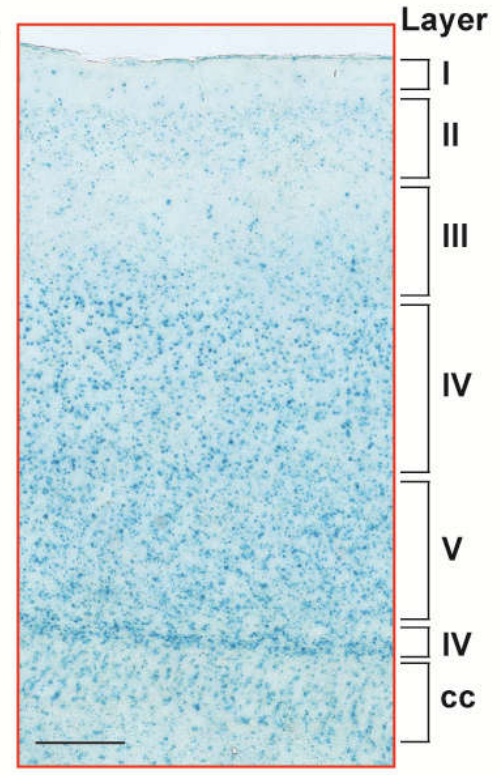

d

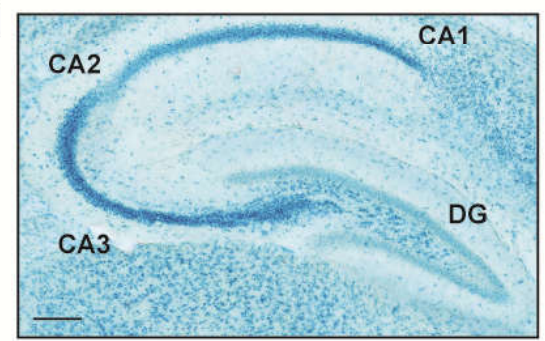

f

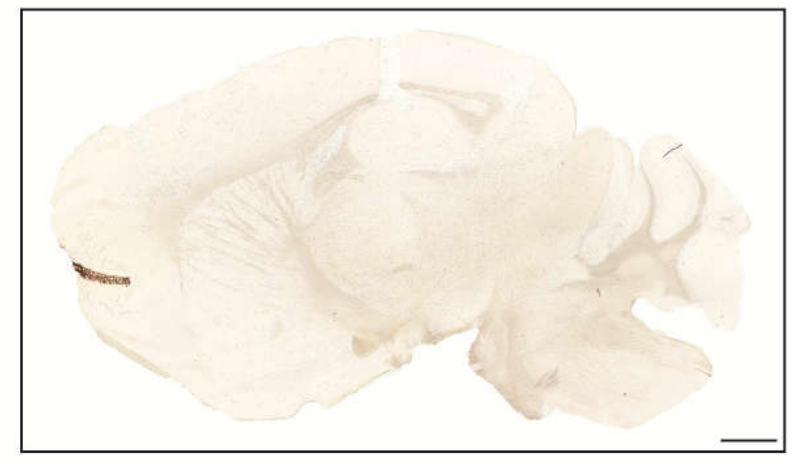


cytoplasm in neurons

Figure 2.2: FBXO7 expression in the murine brain: (a, b) $40 \mu \mathrm{m}$ thick sagittal sections at lateral $0.36 \mathrm{~mm}$ and $2.04 \mathrm{~mm}$, respectively, of $F B X O 7-/-$ mice were subjected to lacZ staining. $\mathrm{Cb}=\mathrm{cerebellum}$, $\mathrm{cc}=\mathrm{corpus}$ callosum, $\mathrm{Ctx}=$ cortex, $\mathrm{Hpc}=$ hippocampus, $\mathrm{M}=$ medulla oblongata, Olf= olfactory bulb, $\mathrm{Pn}=$ pontine nucleus, $\mathrm{Th}=$ thalamus, $\mathrm{Sc}=$ superior colliculus, Str= striatum. Scale bars equal $1000 \mu \mathrm{m}$. (c) Inset represents a higher magnification picture of (a) showing the cortical layers I-VI and the cc. Scale bars equal $200 \mu \mathrm{m}$. (d) Inset represents a higher magnification picture of (a) showing the hippocampal formation. CA1-3= cornu ammonis $1-3, D G=$ dentate gyrus. Scale bar equals $200 \mu \mathrm{m}$. (e) Inset represents a higher magnification picture of (b) showing the cerebellum. $P C L=$ Purkinje cell layer, $I G L=$ internal granular layer, $W M=$ white matter. Scale bar equals $200 \mu \mathrm{m}$. (f) $40 \mu \mathrm{m}$ thick sagittal section of a FBXO7 +/+ mouse subjected to lacZ staining serving as a negative control with no $\beta$-Galactosidase reactivity. Scale bar equals $1000 \mu \mathrm{m}$.

Having established the expression of FBXO7 in the mouse brain, I investigated the subcellular localization in neurons by first carrying out a crude subcellular fractionation analysis. Immunoblotting using the FBXO7 antibody revealed that FBXO7 robustly localized to the cytoplasmic fraction and was absent from the nuclear fraction in P18 mouse cortical tissue (Figure 2.3 a). Furthermore, due to the lack of a specific antibody for detection of endogenous FBXO7 by immunocytochemistry, I resorted to transfection of rat cultured hippocampal neurons with a GFP-tagged FBXO7 plasmid. GFP-FBXO7 localized to the cytoplasm as well as the neurites but not to the nucleus, further confirming FBXO7 as a cytotplasmic protein in neurons (Figure 2.3 b).

a

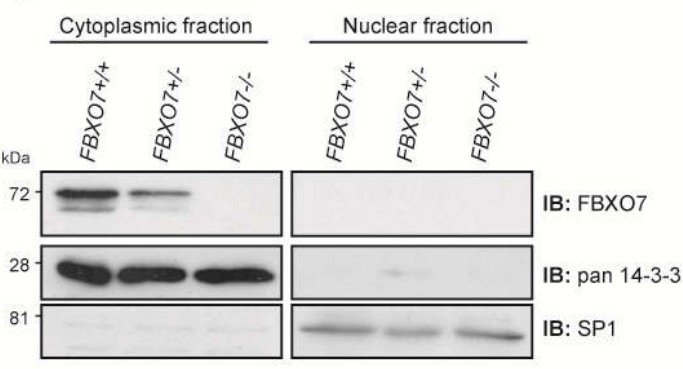

b

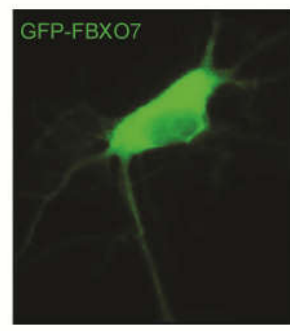

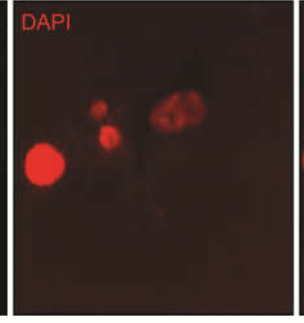

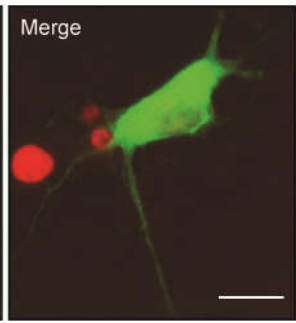

Figure 2.3: FBXO7 localizes to the cytoplasm in neurons: (a) Cortical lysates of $\mathrm{P} 18 \mathrm{FBXO7+/+ \text {, }}$ $F B X O 7+/$ - and $F B X O 7-/-$ mice were subjected to subcellular fractionation, followed by immunoblotting with the FBXO7 antibody. 14-3-3 and SP1 served as cytoplasmic and nuclear marker, respectively. (b) Fluorescent microscopy pictures of cultured hippocampal neurons transfected with GFP-FBXO7 expressing plasmid, followed by immunocytochemistry using the GFP antibody and the DNA-binding dye DAPI. 


\subsection{No a-synuclein- or amyloid precursor protein (APP)-positive inclusions in the brain of $F B X O 7-/-$ mice}

Ubiquitin- and a-synuclein-positive inclusions are hallmarks of sporadic PD but their prevalence in genetic cases of the disease and the reproducibilty in PD mouse models are variable (Hirsch, 2007; Poulopoulos et al., 2012). To find out whether the FBXO7-/- mouse displays any $\alpha$-synuclein abnormalities in the brain, I subjected sagittal brain sections of P18 $F B X O 7+/+$ and FBXO7-/- mice to immunohistochemistry using the a-synuclein antibody. Screening of the $F B X O 7-/-$ mouse brain sections revealed no a-synuclein-positive inclusions (Figure 2.4 a). Additionally, I checked a-synuclein protein levels in P18 mouse cortical lysates and found no change (Figure 2.4 b, c). Owing to FBXO7's expression in oligodendrocytes, I wondered whether the axonal integrity on a lightmicroscopical level was intact. To test this, I subjected sagittal brain sections of P18 FBXO7+/+ and FBXO7-/- mice to immunohistochemistry using the APP antibody as APP is prone to accumulate as a result of axonal damage (Trapp et al., 1998). Screening of the white matter regions of the corpus callosum and the cerebellar white matter revealed no APP-positive inclusions (Figure $2.4 \mathbf{d}$ ). Also, APP protein levels in P18 FBXO7-/- mouse cortical lysates were unaltered when compared to the wild type littermates (Figure $2.4 \mathrm{e}, \mathbf{f}$ ). 
Results

2.3 No $\alpha$-synuclein- or amyloid precursor protein-positive inclusions in the brain of FBXO7-/- mice

a
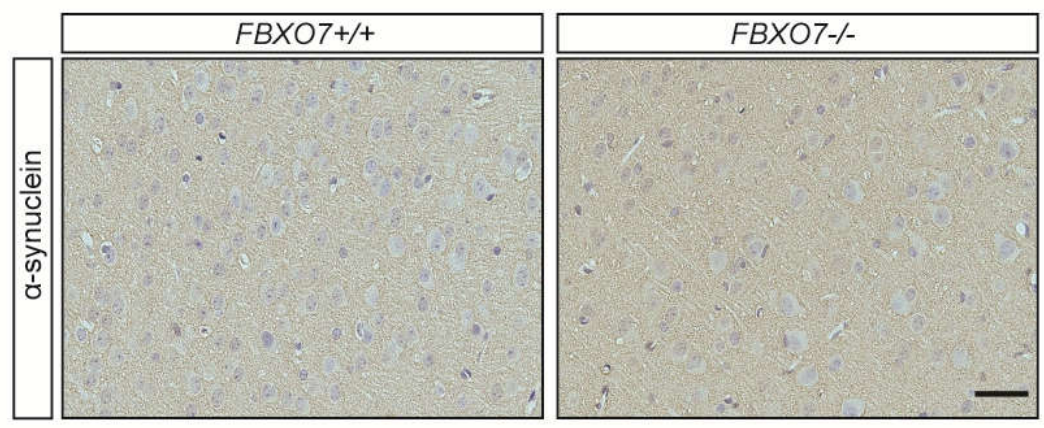

b
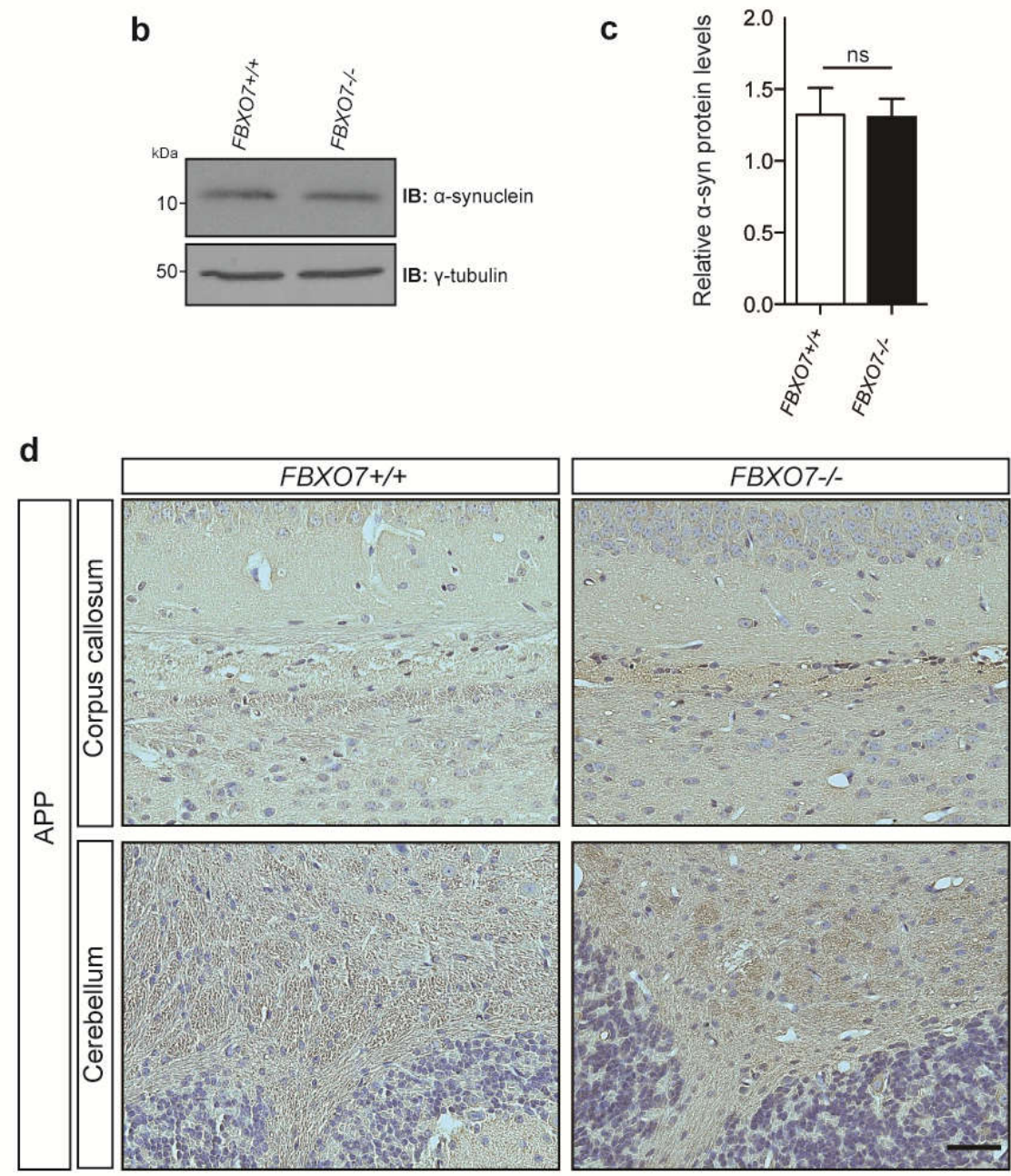

e
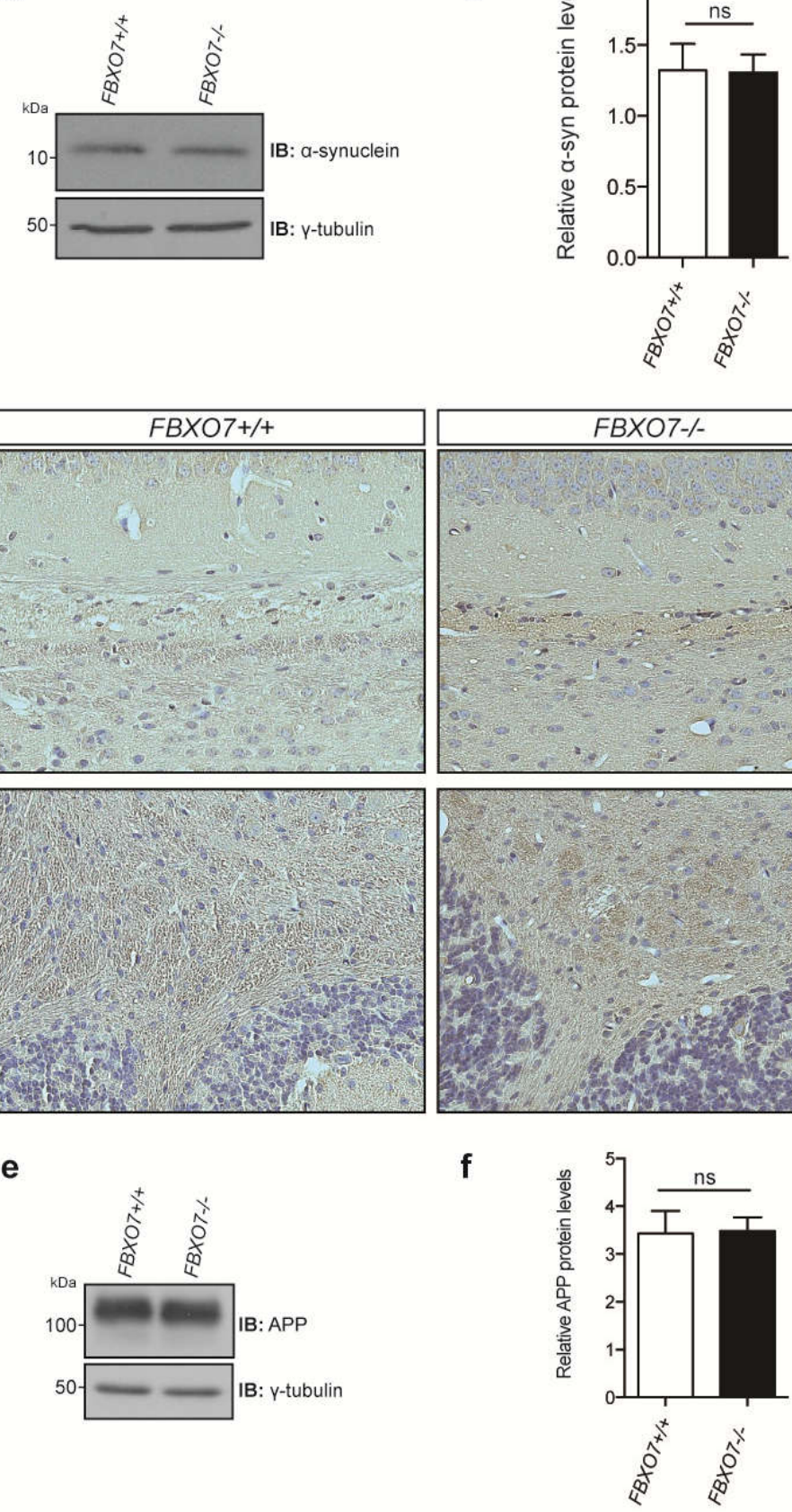


\subsection{No $\alpha$-synuclein- or amyloid precursor protein-positive inclusions in the

Figure 2.4: FBXO7-/- mouse brains display no $\alpha$-synuclein- or APP-positive inclusions: (a) Representative images from $5 \mu \mathrm{m}$ thick sagittal paraffin-embedded brain sections of the cortical region of $\mathrm{P} 18$ FBXO7+/+ and FBXO7-/- mice. Whole brain sections were subjected to immunohistochemistry using the asynuclein antibody and screened for inclusions. Scale bar equals $40 \mu \mathrm{m}$. (b) Cortical lysates of P18 $\mathrm{FBXO7+/+}$ and FBXO7-/- mice were subjected to immunoblotting using the $\alpha$-synuclein and $\mathrm{y}$-tubulin antibodies. The latter served as a loading control. (c) Densitrometric quantification of $\alpha$-synuclein protein levels in (b). n= 5 FBXO7 litterpairs were analyzed (Paired t-test, ns= non significant, mean + s.e.m.). (d) Representative images from $5 \mu \mathrm{m}$ thick sagittal paraffin-embedded brain sections of the corpus callosum and cerebellar white matter region of P18 FBXO7+/+ and FBXO7-/- mice. Whole brain sections were subjected to immunohistochemistry using the APP antibody and screened for inclusions. Scale bar equals $40 \mu \mathrm{m}$. (e) Cortical lysates of P18 FBXO7 +/+ and FBXO7 -/- mice were subjected to immunoblotting using the APP and $\mathrm{Y}$-tubulin antibodies. The latter served as a loading control. (f) Densitrometric quantification of APP protein levels in (e). n= 8 FBXO7 litterpairs were analyzed (Paired t-test, ns= non significant, mean + s.e.m.).

\subsection{Increased astrogliosis and elevated levels of apoptosis in the brain of $F B X 07-/-$ mice}

Increased inflammation is associated with several neurodegenerative diseases, including Parkinson's disease (Damier et al., 1993; Dickson, 2012). I hence compared sagittal brain sections of P18 FBXO7+/+ and FBXO7-/- mice first for astrogliosis using the glial fibrillary acidic protein (GFAP) antibody. Indeed, GFAP levels were increased in the FBXO7-/animals (Figure 2.5 a, b). I also examined for increased microgliosis using the lba1 antibody on the same brain sections but found no difference (Figure $2.5 \mathrm{c}, \mathrm{d}, \mathrm{e}$ ). 
a

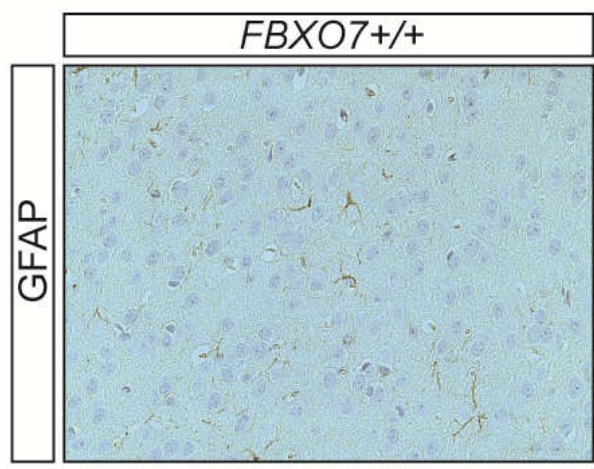

b

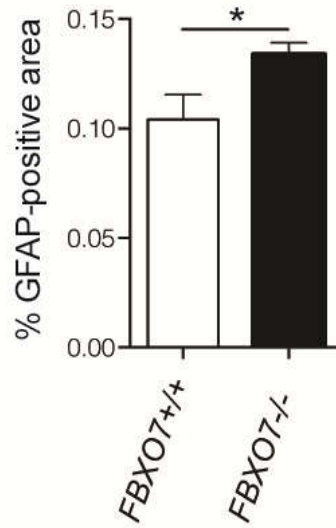

C
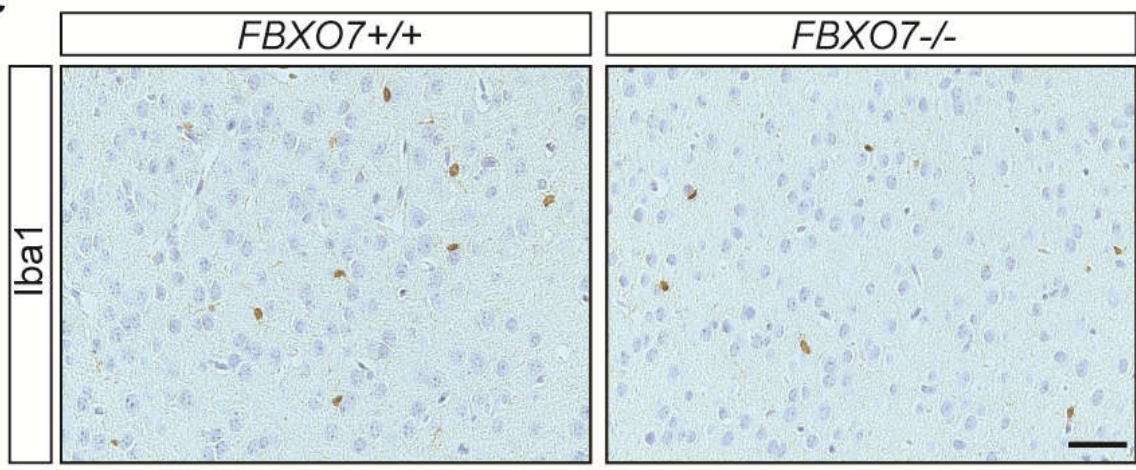

d
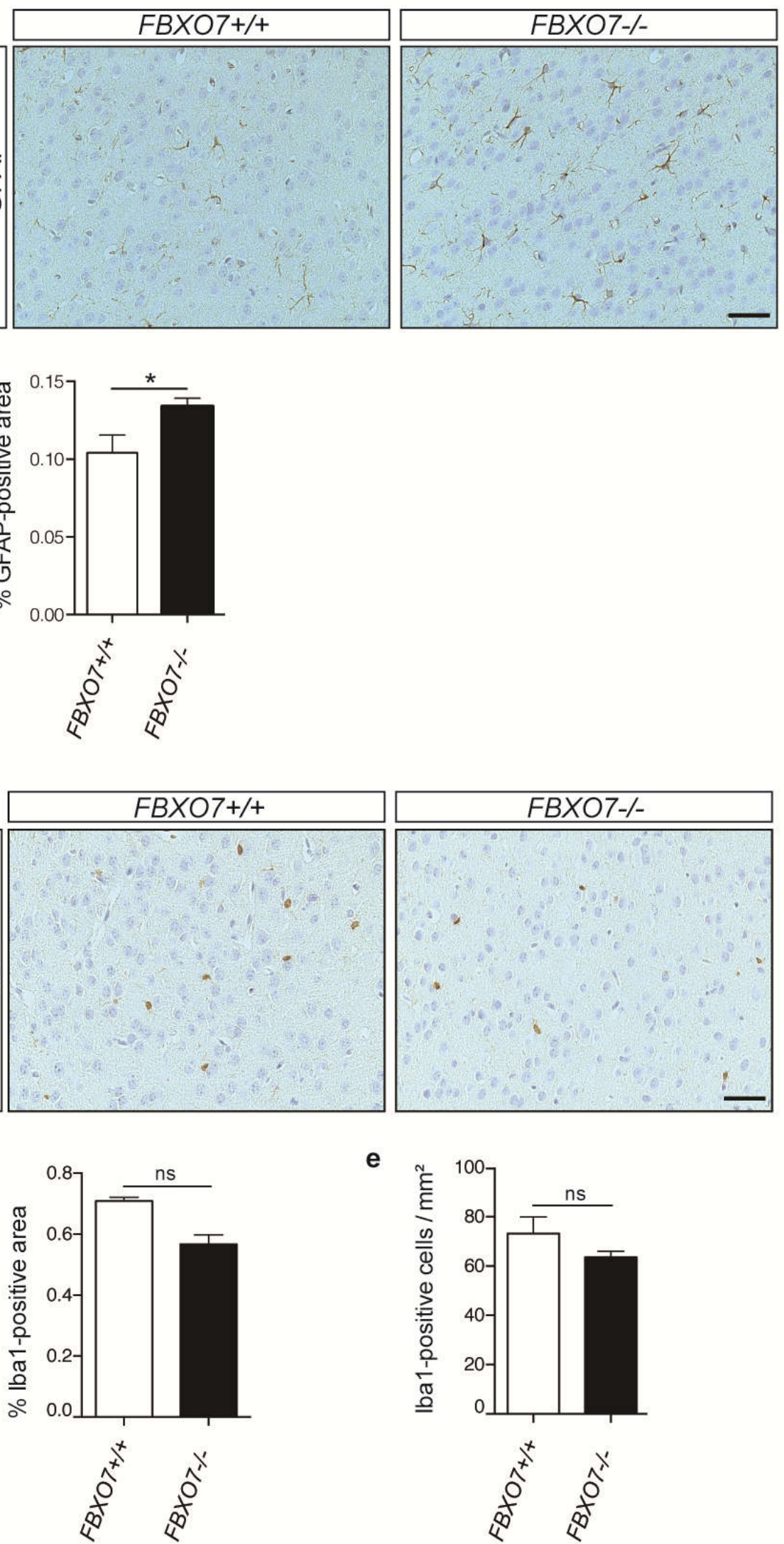

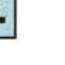


FBX07-/- mice

Figure 2.5: $F B X 07-/-$ mice show increased levels of astrogliosis in the brain: (a) Representative images from $5 \mu \mathrm{m}$ thick sagittal paraffin-embedded brain sections of the cortical region of P18 FBXO7+/+ and FBXO7/- mice. Whole brain sections were subjected to immunohistochemistry using the GFAP antibody. Scale bar equals $40 \mu \mathrm{m}$. (b) Quantification of GFAP-positive area (\% stained area/mm $\mathrm{mm}^{2}$ ) in the cortex of at least three independent $\mathrm{FBXO7+/+}$ and FBXO7-/- littermates. Three anatomically matched sections per animal were quantified in a blinded manner and analyzed using a custom-designed macro. (Paired t-test, ${ }^{*} p<0.05$, mean + s.e.m.). (c) Representative images from $5 \mu \mathrm{m}$ thick sagittal paraffin-embedded brain sections of the cortical region of $\mathrm{P} 18 \mathrm{FBXO} 7+/+$ and $\mathrm{FBXO} 7$-/- mice. Whole brain sections were subjected to immunohistochemistry using the Iba1 antibody. Scale bar equals $40 \mu \mathrm{m}$. (d) Quantification of lba1-positive area (\% stained area $/ \mathrm{mm}^{2}$ ) in the cortex of at least three independent FBXO7 +/+ and FBXO7 -/- littermates. Three anatomically matched sections per animal were quantified in a blinded manner and analyzed using a customdesigned macro. (Paired t-test, ns= non significant, mean + s.e.m.). (e) Quantification of Iba1-positive cells (cells $/ \mathrm{mm}^{2}$ ) of the same brain sections quantified in (d). Quantification was done manually and in a blinded manner. (Paired t-test, ns= non significant, mean + s.e.m.).

Cell death and apoptosis are major factors in neurodegenerative diseases and are usually accompanied by inflammation (Wyss-Coray and Mucke, 2002). I hence determined, whether brain sections of P18 FBXO7+/+ and FBXO7-/- mice showed any abnomality in cell death levels using a commercial TUNEL kit. The TUNEL kit detects sites of fragmented DNA that result from apoptotic pathways. Interestingly, many of the brain regions analyzed (cortex, cerebellum, hippocampus) showed a tendency to elevated levels of apoptotic cells being only in the cortex significantly increased (Figure 2.6 a, b). Levels of apoptotic cells in the mibrain

(Figure 2.6 c, d) as well as the number of dopaminergic in the substantia nigra were unchanged (Data Siv Vingill). 
a

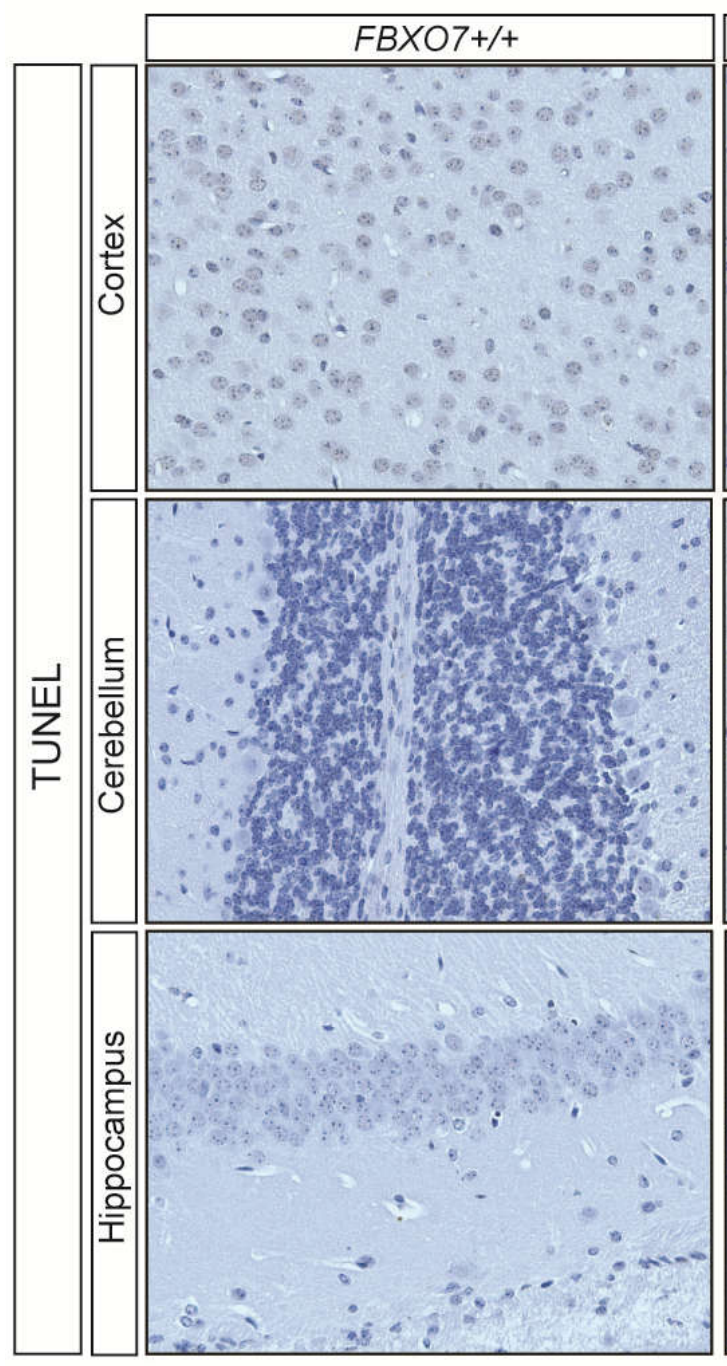

b
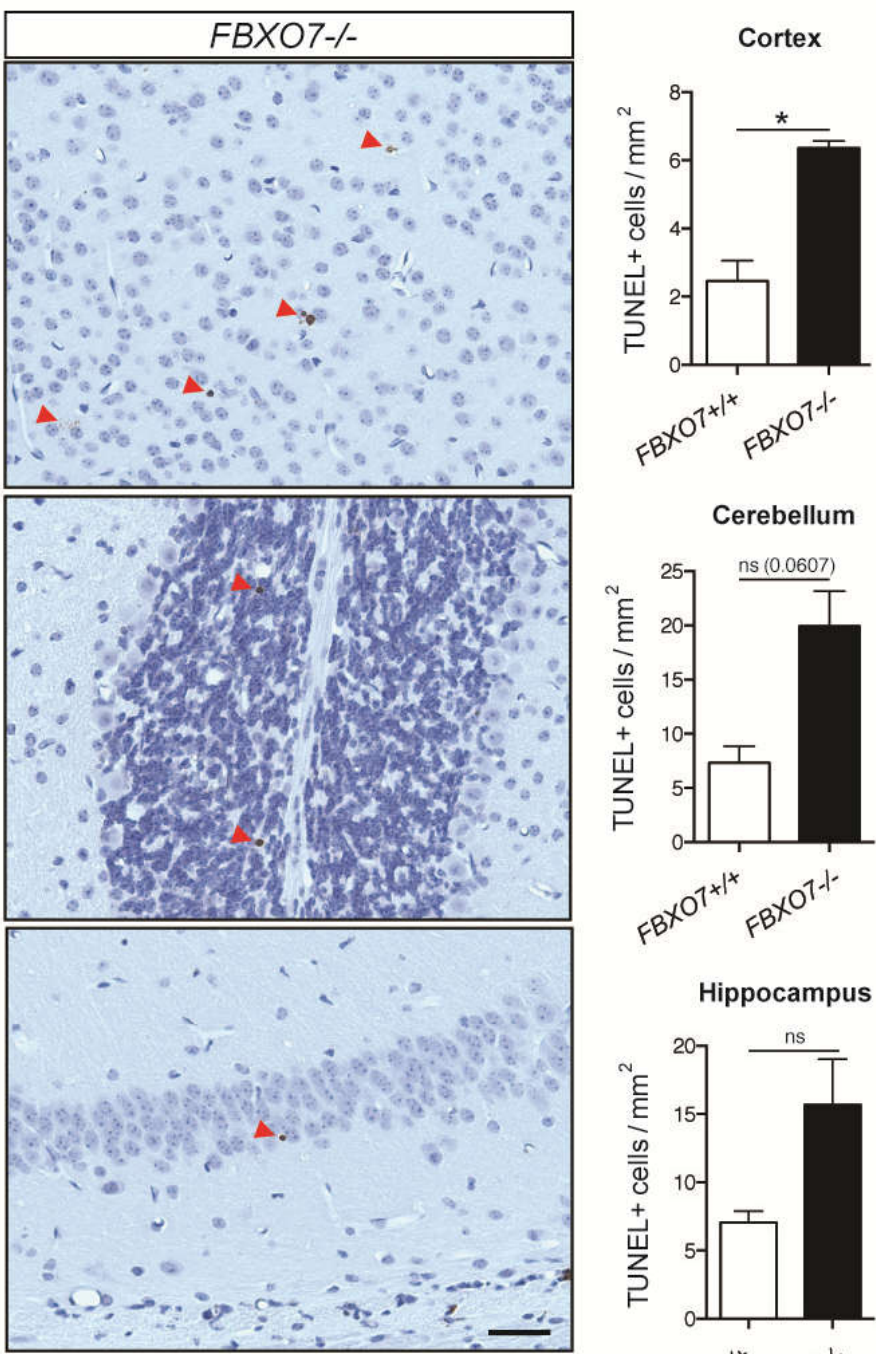

Hippocampus

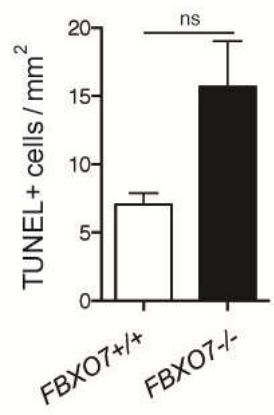

d

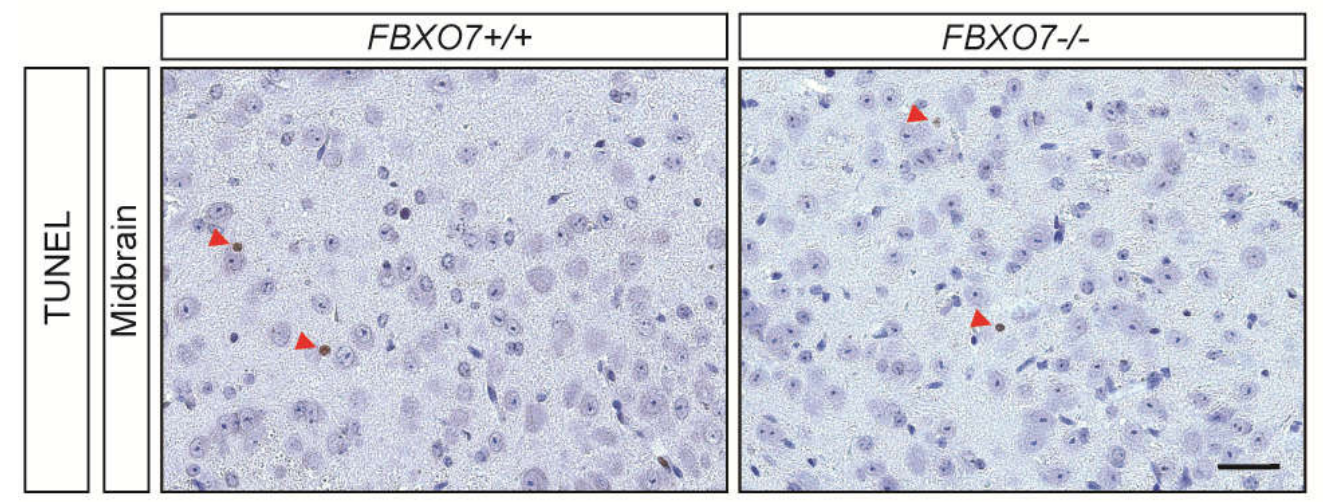

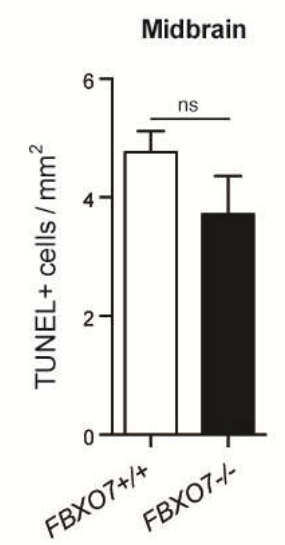


FBX07-/- mice

Figure 2.6: FBXO7-/- mice display increased apoptosis in the brain: (a) Representative images from $5 \mu \mathrm{m}$ thick sagittal paraffin-embedded brain sections of the cortical, cerebellar and hippocampal region of $\mathrm{P} 18$ $F B X O 7+/+$ and FBXO7-/- mice. Whole brain sections were subjected to immunohistochemistry using a TUNEL detection kit, staining apoptotic cells. Arrowheads indicate apoptotic cell bodies. Scale bar equals 40 $\mu \mathrm{m}$. (b) Quantification of TUNEL-positive cells $\left(\right.$ cells $/ \mathrm{mm}^{2}$ ) in the cortex, cerebellum and hippocampus. Three anatomically matched sections per animal were quantified manually in a blinded manner. (Paired t-test, ${ }^{*} \mathrm{p}<0.05$, ns= non significant, mean + s.e.m.). (c) Representative images from $5 \mu \mathrm{m}$ thick coronal paraffinembedded brain sections of the midbrain region of $\mathrm{P} 18 \mathrm{FBXO7+/+}$ and FBXO7-/- mice. Whole brain sections were subjected to immunohistochemistry using a TUNEL detection kit, staining apoptotic cells. Arrowheads indicate apoptotic cell bodies. Scale bar equals $40 \mu \mathrm{m}$. (d) Quantification of TUNEL-positive cells (cells $/ \mathrm{mm}^{2}$ ) in the midbrain. Three anatomically matched sections per animal were quantified manually in a blinded manner. (Paired t-test, ns= non significant, mean + s.e.m.).

To corroborate the increased levels of apoptosis observed in vivo, I performed a survival assay by acutely knocking down FBXO7 in cultured cortical neurons. For this, I transfected cortical neurons at day in vitro (DIV) 3 with plasmids encoding either the control vector, functional FBXO7 shRNA or non-functional shRNA (Figure $2.7 \mathrm{~b}$ ) and quantified the number of pyknotic nuclei and level of cleaved caspase 3 at DIV 7. The number of apoptotic neurons was increased by more than 2.5 fold in the condition, in which FBXO7 was knocked down as compared to control cells (Figure 2.7 a), suggesting that FBXO7 is essential for cortical neuron survival both in vivo and in vitro.

a

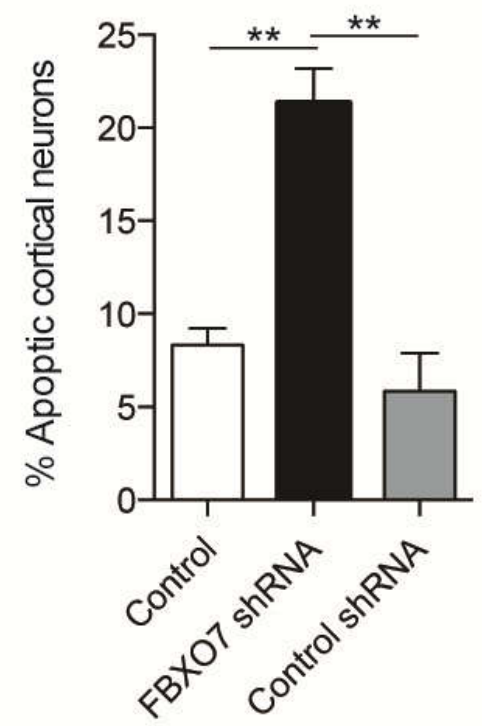

b

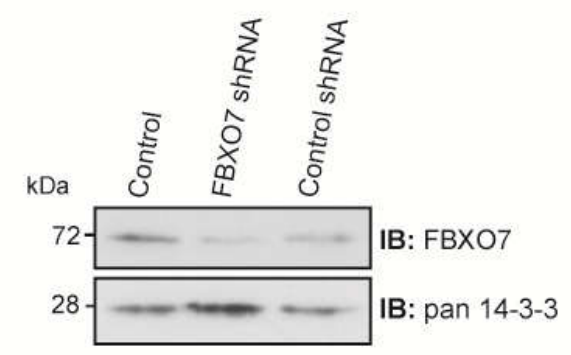

Figure 2.7: FBXO7 is required for neuronal survival: (a) Apoptotic cortical neurons were counted manually in a blind manner and quantified. At least four independent experiments were included in the analysis (ANOVA, ${ }^{* *} p<0.01$, mean + s.e.m.). (b) Lysates from cultured rat cortical neurons were nucleofected at DIV0 with either control pSuper, functional FBXO7 shRNA or non-functional FBXO7 as an additional control. Neurons were harvested four days after nucleofection and subjected to immunoblotting with the FBXO7 and 14-3-3 antibodies. The latter served as a loading control. 


\subsection{Screening for novel FBXO7 interacting proteins}

Since the first reports associated mutations in the FBXO7 gene with hereditary parkinsonism, FBXO7 has been implicated in several cell contexts including cell cycle, mitophagy and the proteasome (Nelson et al., 2013). However, mechanistic insight into how FBXO7 influences these cell functions is very limited and in particular its role as an E3 ligase remains greatly underexplored.

Ubiquitination targets have to directly interact with the E3 ligase. To uncover those, I sought to identify interaction partners of FBX07. For this, I carried out a yeast two-hybrid screen using full-length FBXO7 fused to the DNA-binding domain (DNA-BD) and a human fetal brain cDNA library fused to the activator domain (AD) of the yeast transcription factor Gal4. Both the FBXO7 plasmid and the plasmid library were transformed into different yeast strains, the yeast strains were mated and subsequently selected for compensation of auxotrophy by growth on triple amino acid-lacking medium plates. The selection was based on two amino acid synthesis genes being encoded on either the bait or the prey plasmid. The third selection gene was transactivated by physical proximity of the Gal4 transcription factor DNA-BD and AD upon interaction of FBXO7 with one of the library proteins.

The initial control tests of the yeast two-hybrid screen included investigation of bait toxicity, bait autoactivation and control mating. Normal yeast colony growth of the tryptophane-auxotrophic yeast AH109 transformed with pGBT9-BD-FBXO7 on SD/-Trp plates showed that no bait toxicity was present. To exclude that the bait construct transactivates the third selection gene without the prey construct, yeast growth of pGBT9-BD-FBXO7 transformed into $\mathrm{AH} 109$ yeast on SD/-His plates was tested and resulted in no colony growth. The control mating between the transformed $\mathrm{AH} 109$ and $\mathrm{Y} 187$ yeast strains yielded stable growth on SD/-Trp/-Leu indicating that yeast mating had taken place.

After successful small-scale control mating, the large-scale mating for library screening was carried out by growth selection of the transformed yeast strains on SD/-Trp/Leu/-His plates. Subsequently, library titer, diploid number and mating efficiency were determined as verification factors for the screen. The library titer was determined to be $1.24 \mathrm{x}$ $10^{8}$ and was in the range of expected values ( $>2 \times 10^{7}$ cells). The diploid number counts indicated that a total of $14.6 \times 10^{6}$ clones were screened, which is well above the recommended $1 \times 10^{6}$ for efficient screening. Mating efficiency was determined by the ratio of viability of diploids to the viability of the prey library and was found to be $12.3 \%$, which was 
exceeding the expected $2-5 \%$. The verification factors indicated that the screen was efficiently carried out.

Approximately 175 yeast colonies grown on the triple selection plates were then subjected to a ß-galactosidase assay to exclude false-positives followed by colony PCR and sequencing for identification of the putative interactor. Among the identified interaction partners were already published interactors, such as PI31 (Kirk et al., 2008) and SKP1 (Hsu et al., 2004) (Table 2.1). Interestingly, two novel putative interactors were identified - the proteasomal subunit alpha 2 (PSMA2) and the light chain of the microtubule-associated protein $1 \mathrm{~B}$ (MAP1B-LC1) (Table 2.1).

Table 2.1: List of identified interaction partners by yeast two-hybrid screening.

\begin{tabular}{c|cc|} 
Putative interactor & Number of hits & Status \\
\hline PI31 & 34 & Known interactor (Kirk et al., 2008) \\
SKP1 & 1 & Known interactor (Hsu et al., 2004) \\
PSMA2 & 13 & Unknown interactor \\
MAP1B-LC1 & 2 & Unknown interactor \\
\cline { 2 - 3 }
\end{tabular}

\subsection{FBX07 interacts with members of the MAP1 (microtubule- associated protein) familiy}

MAP1A, MAP1B and MAP1S are microtubule-associated proteins. While MAP1S is a comparably small (120 kDa) protein with ubiquitous expression (Liu and McKeehan, 2002), MAP1A and MAP1B are larger proteins (> $300 \mathrm{kDa}$ ) and only expressed in the central and peripheral nervous system (Noiges et al., 2002; Togel et al., 1998). All three proteins are synthesized as polyprotein precursors that are subsequently cleaved into a heavy and light chain. The two chains can subsequently reassociate or exert separate functions (Schoenfeld and Obar, 1994). Having identified the light chain 1 of MAP1B as a potential interactor in the yeast two-hybrid screen, I went on to confirm MAP1B-LC1 as an interactor in a mammalian cell system. Transfection of HEK293T cells with plasmids encoding control vector, mycFBXO7 and FLAG-LC1 followed by co-immunoprecipitation analyses with either of the possible antibody combinations revealed the specific interaction of FBXO7 and MAP1B-LC1 (Figure 2.8 a, b, c). 
a

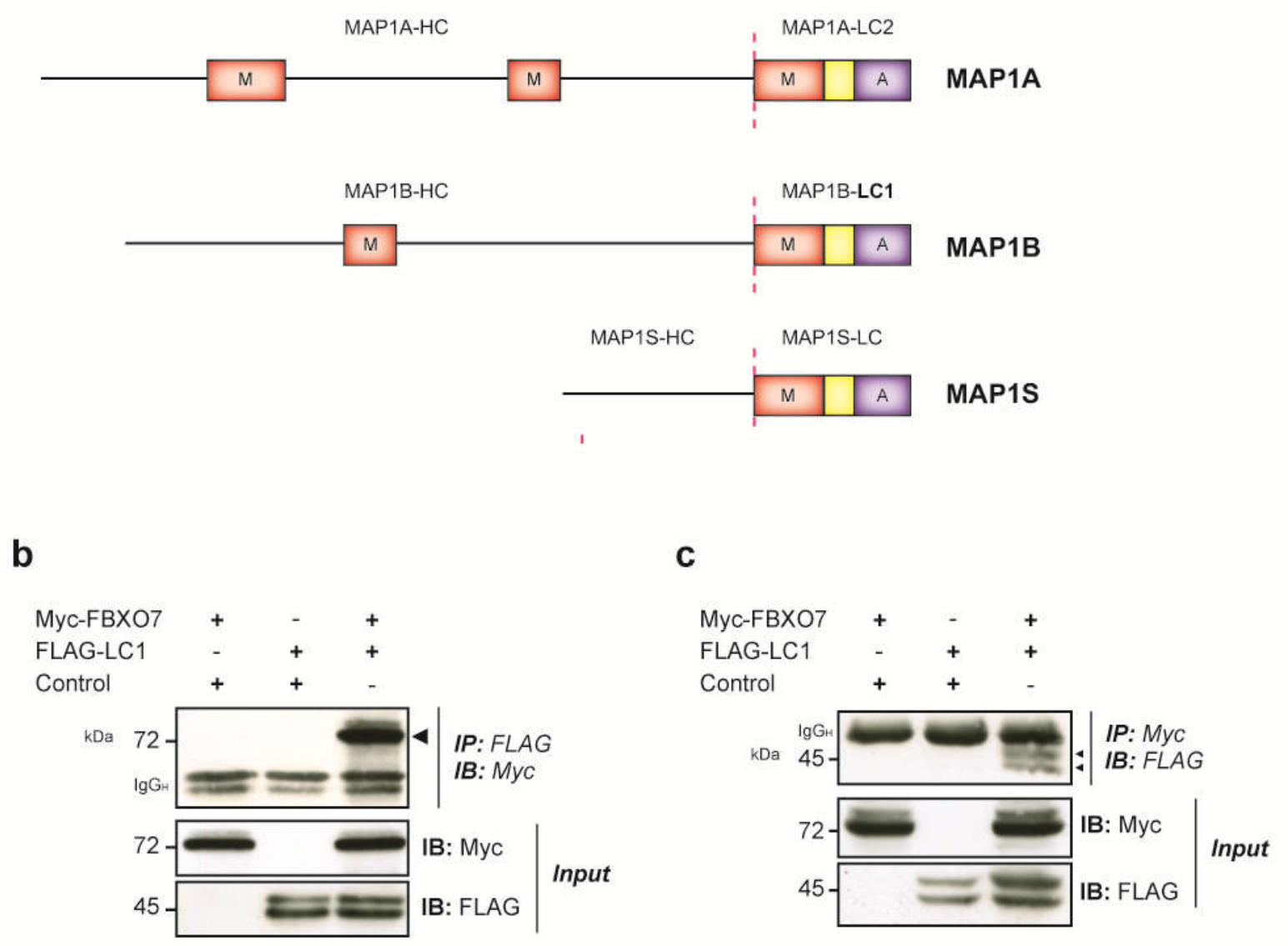

Figure 2.8: FBXO7 interacts with MAP1B-LC1: (a) Schematic representing the MAP1 protein familiy. Dashed line represents the cleavage site between heavy chain (HC) and light chain (LC). M= Microtubulebinding domain, $A=$ Actin-binding domain, Yellow box= highly similar MAGD domain. Adapted from \{genome biol, 2006, halpain\} (b) At least $1 \mathrm{mg}$ of lysates from HEK293T cells, transfected with either myc-FBXO7 or FLAG-LC1 together with appropriate control vectors or both myc-FBXO7 and FLAG-LC1 plasmids, were subjected to IP (immunoprecipitation) with the FLAG antibody and subsequent immunoblotting using the myc antibody. $50 \mu \mathrm{g}$ of total cell lysate was subjected to IB (immunoblotting) using the same antibodies and served as an input control. Arrowhead indicates specific interaction band. IgGH indicates the heavy chain of the IP antibody. (c) At least $1 \mathrm{mg}$ of lysates from HEK293T cells transfected with either myc-FBXO7 or FLAG-LC1 together with appropriate control vectors or both myc-FBXO7 and FLAG-LC1 plasmids were subjected to IP with the myc antibody and subsequent immunoblotting using the FLAG antibody. $50 \mu \mathrm{g}$ of total cell lysate was subjected to immunoblotting using the same antibodies and served as an input control. Arrowhead indicates specific interaction band. IgGH indicates the heavy chain of the IP antibody.

To investigate which domain of FBXO7 was responsible for LC1-binding, I carried out a mapping analysis using FBXO7 deletion mutants (Figure 2.9 a). Co-immunoprecipitation analyses using transfected HEK293T cells with the control vector or the myc-FBXO7 deletion mutants together with FLAG- MAP1B-LC1 identified the amino acids 1 - 180, which include the ubiquitin-like (Ubl) domain, as indispensable for the interaction (Figure 2.9 b). I furthermore investigated which of the known domains on MAP1B-LC1 was responsible for the interaction with FBXO7 and generated three LC1 deletion mutants (Figure 2.9 c). Using 


\subsection{FBXO7 interacts with members of the MAP1 family}

HEK293T cells transfected with the control vector or either of the FLAG-LC1 deletion mutants along with myc-FBXO7 identified the 25-amino acid mitochondrial aggregation and genome destruction (MAGD) domain (Liu et al., 2005) as the specific region of interaction (Figure 2.9

d).

a

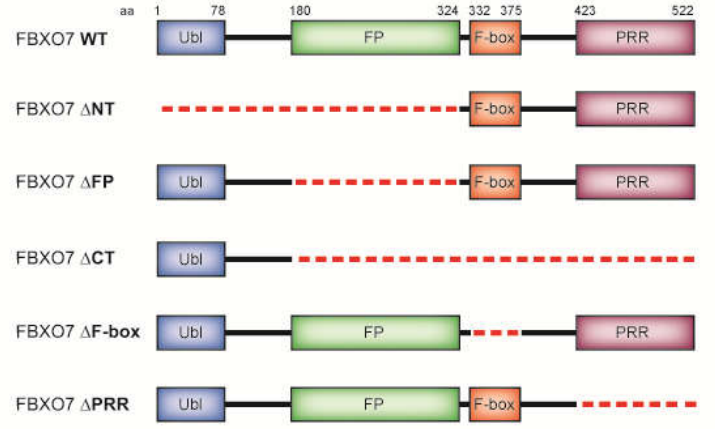

C

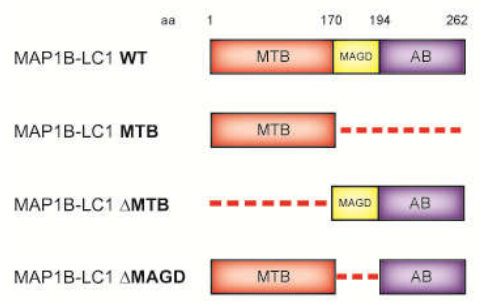

b
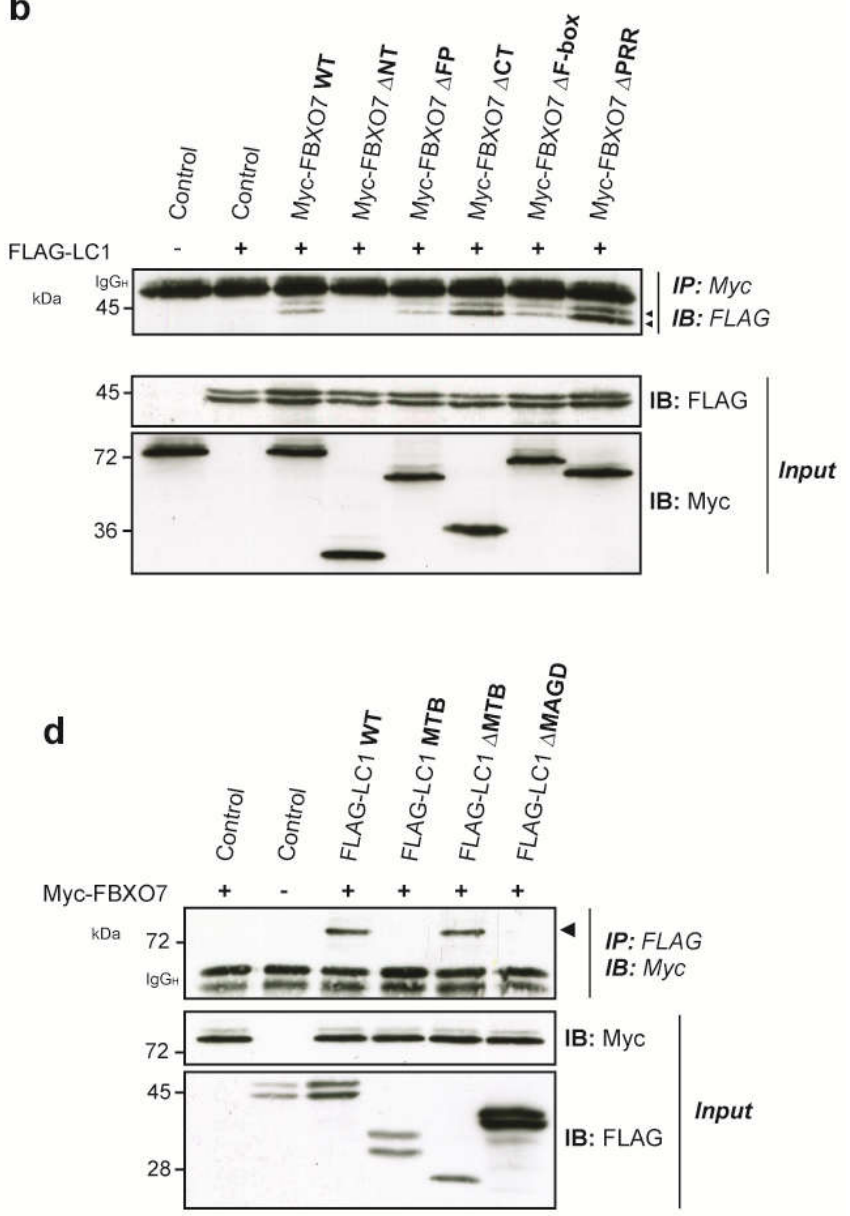

Figure 2.9: Mapping analysis of the FBXO7-LC1 interaction: (a) Schematic representation of FBXO7 deletion ( ) mutants. WT= wild type, NT= N-terminus, FP= FBXO7-PI31 interaction domain, CT= C-terminus, F-box= F-box domain, PRR= proline-rich region. (b) At least $1 \mathrm{mg}$ of lysates from HEK293T cells, transfected with either FLAG-LC1 or myc-FBXO7 with respective control vectors or FLAG-LC1 together with either the myc-FBXO7 WT, NT, FP, CT, F-box or PRR plasmid were subjected to IP with the myc antibody and subsequent IB using the FLAG antibody. $50 \mu \mathrm{g}$ of total cell lysate was subjected to IB using the same antibodies and served as input control. Arrowheads indicate specific interaction bands. IgGH indicates the heavy chain of the IP antibody. (c) Schematic representation of MAP1B-LC1 mutants. WT= wild type, MTB= Microtubule-binding domain only, MTB= Lacking the microtubule-binding domain, MAGD= Lacking the mitochondrial aggregation and genome destruction, $A B=$ Actin-binding domain (d) At least $1 \mathrm{mg}$ of lysates from HEK293T cells, transfected with either myc-FBXO7 or FLAG-LC1 with respective control vectors or mycFBXO7 together with either FLAG-LC1, MTB, MTB or MAGD were subjected to IP with the FLAG antibody and subsequent IB using the myc antibody. $50 \mu \mathrm{g}$ of total cell lysate was subjected to IB using the same antibodies and served as an input control. Arrowhead indicates specific interaction band. IgGH indicates the heavy chain of the IP antibody. 


\subsection{FBXO7 interacts with members of the MAP1 family}

Since this domain displays high sequence similarity between LC1 of MAP1B, LC2 of MAP1A and MAP1S, I wondered whether LC2 and MAP1S would also interact with FBXO7. Co-immunoprecipitation analyses of HEK293T cells transfected with control or myc-FBXO7 along with either FLAG-LC2 or full-length FLAG-MAP1S identified two more interactors of FBXO7 (Figure 2.10 a, b) and furthermore elucidates the MAGD region as the specific FBXO7-binding motif.

a

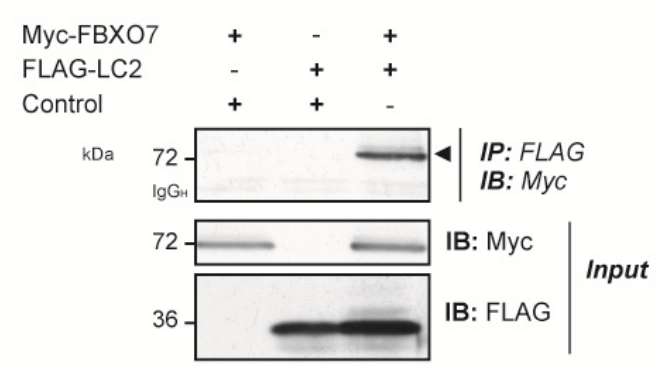

b

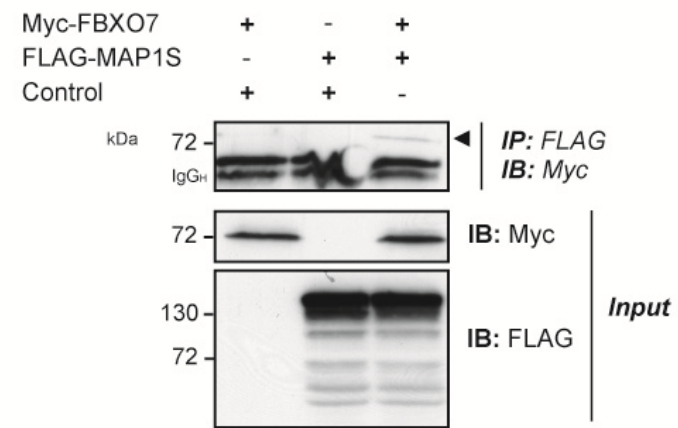

Figure 2.10: FBXO7 also interacts with the MAP1 family members MAP1A-LC2 and MAP1S: (a) At least $1 \mathrm{mg}$ of lysates from HEK293T cells, transfected with either myc-FBXO7 or FLAG-LC2 together with appropriate control vectors or myc-FBXO7 and FLAG-LC2 together were subjected to IP with the FLAG antibody and subsequent IB using the myc antibody. $50 \mu \mathrm{g}$ of total cell lysate was subjected to IB using the same antibodies and served as an input control. Arrowhead indicates specific interaction band. IgGH indicates the heavy chain of the IP antibody. (b) At least $1 \mathrm{mg}$ of lysates from HEK293T cells, transfected with either myc-FBXO7 or FLAG-MAP1S with respective control vectors or myc-FBXO7 and FLAG-MAP1S together were subjected to IP with the FLAG antibody and subsequent IB using the myc antibody. $50 \mu \mathrm{g}$ of total cell lysate was subjected to IB using the same antibodies and served as an input control. Arrowhead indicates specific interaction band. IgGH indicates the heavy chain of the IP antibody. 
FBXO7-SCF

\subsection{The MAP1 family proteins are potential ubiquitination substrates of FBX07-SCF}

Having established MAP1B-LC1, MAP1A-LC2 and MAP1S as novel FBXO7 interaction partners, I asked whether these proteins could be potential ubiquitination substrates of FBX07. Co-expression analyses of control, myc-FBX07 WT or myc-FBXO7 Fbox (a mutant lacking the E3 ligase activity-conferring domain) with FLAG-LC1 or FLAG-LC2 resulted in the appearance of a higher molecular weight smear that was sensitive to ligase activity of FBXO7 (Figure 2.11 a, c brackets) suggesting that it might be ubiquitin modification. This effect was specific to $\mathrm{FBXO7}$, since co-expression of another F-box protein FBXO31 did not result in the same effect (Figure 2.11 b, d). Furthermore, co-expression of HA-tagged ubiquitin enhanced the intensity of the higher molecular weight signals on FLAGLC2 indicating that the observed modification might be ubiquitination (Figure $2.11 \mathrm{e}$ ). As compared to wild type ubiquitin, a ubiquitin mutant that was unable to form lysine (K) 63linked chains diminished the appearance of the smear whereas another ubiquitin variant harboring the K48R mutation did not diminish the smear (Figure $2.11 \mathrm{e}$ ). As compared to LC2, LC1 appeared as a double band in the co-expression analysis independent of FBXO7. To investigate, whether one of the bands was a phosphorylated form of LC1, I treated transfected HEK293T lysates with $\lambda$-protein phosphatase. Indeed, the upper band of FLAGLC1 was responsive to $\lambda$-protein phosphatase treatment while FBXO7 did not show any response (Figure 2.11 f). SP1 served as a positive control and displayed a shift indicative of a loss of phosphorylation. Taken together, I confirmed MAP1B-LC1, MAP1A-LC2 and MAP1S as novel interaction partners of FBXO7 and furthermore presented data suggesting that LC1 and LC2 could be potential ubiquitination substrates of FBXO7. Further experiments, such as in vivo or in vitro ubiquitination assays are required to confirm this hypothesis. 

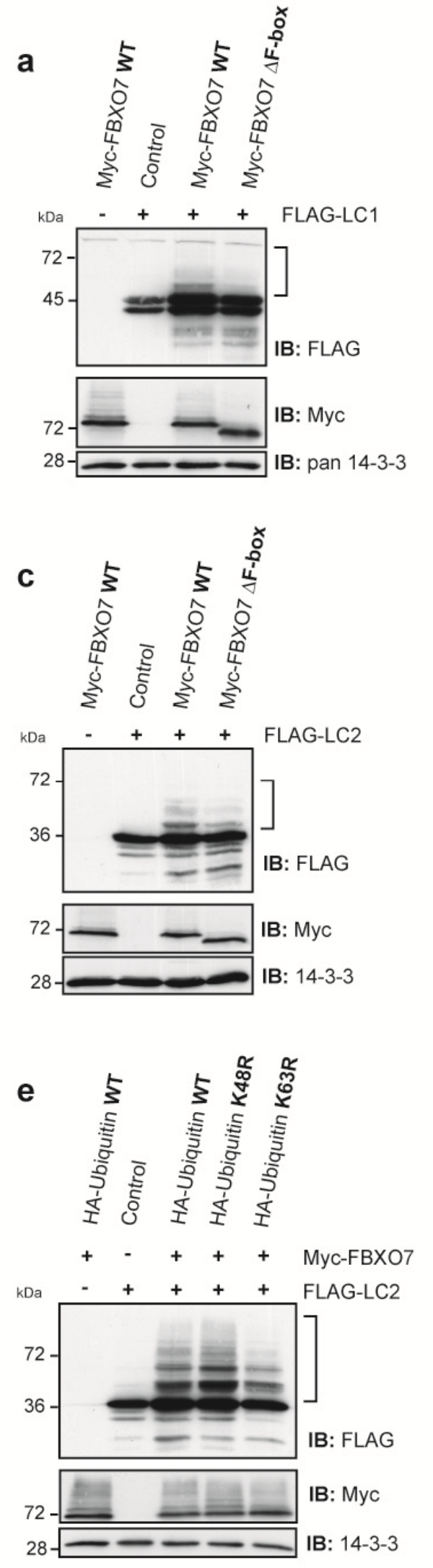

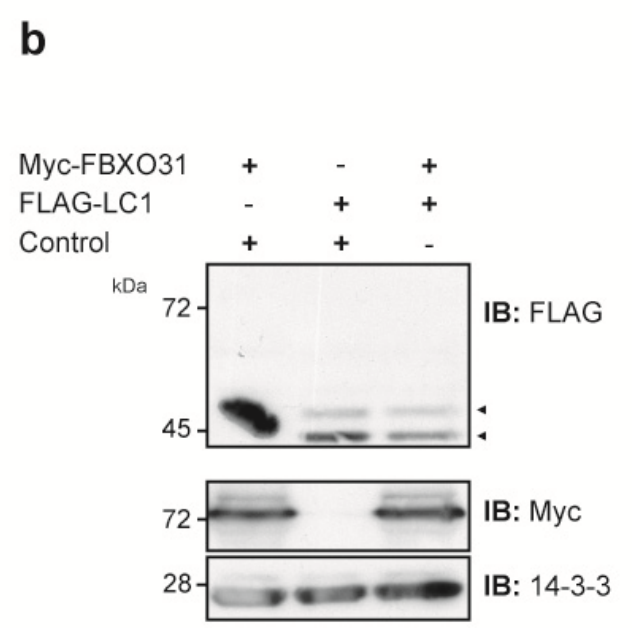

d

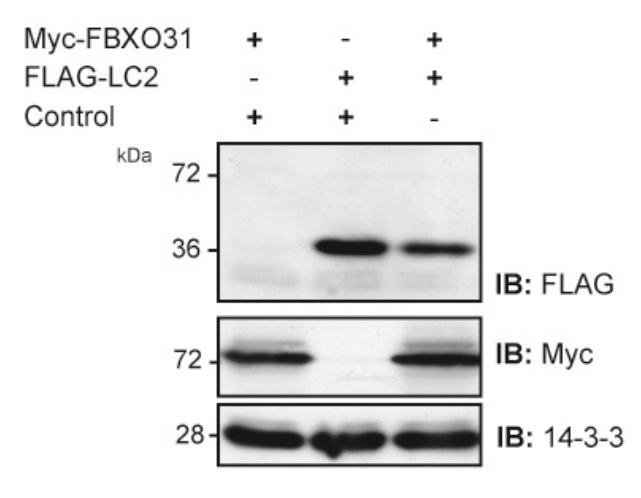

f

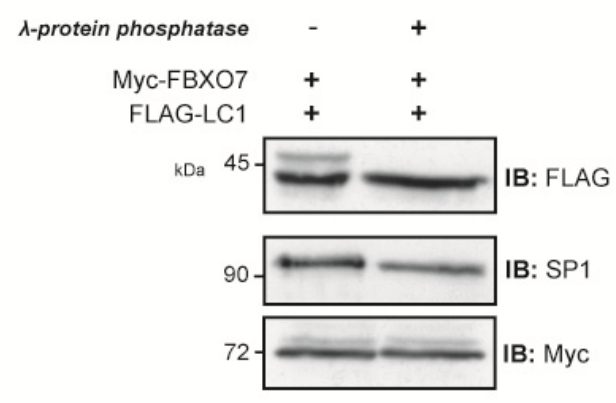


Figure 2.11: LC1 and LC2 proteins are modified in a FBX07-dependent manner: (a) Lysates from HEK293T cells, transfected with either myc-FBXO7 or FLAG-LC1 together with appropriate control vectors as well as FLAG-LC1 together with either myc-FBXO7 WT or F-box plasmid, were subjected to IB using the FLAG, myc or 14-3-3 antibodies, respectively. The latter served as a loading control. The bracket indicates smear. (b) Lysates from HEK293T cells, transfected with either myc-FBXO31 or FLAG-LC1 together with appropriate control vectors as well as FLAG-LC1 together with myc-FBXO31, were subjected to immunoblotting using the FLAG, myc or 14-3-3 antibodies, respectively. The latter served as a loading control. Arrowheads indicate the LC1 protein bands. (c) Lysates from HEK293T cells, transfected with either mycFBXO7 or FLAG-LC2 with respective control vectors as well as FLAG-LC2 together with either myc-FBXO7 WT or F-box, were subjected to immunoblotting using the FLAG, myc or 14-3-3 antibodies, respectively. The latter served as a loading control. The bracket indicates smear. (d) Lysates from HEK293T cells, transfected with either myc-FBXO31 or FLAG-LC2 with respective control vectors as well as FLAG-LC2 together with myc-FBXO31, were subjected to IB using the FLAG, myc or 14-3-3 antibodies, respectively. The latter served as a loading control. (e) Lysates from HEK293T cells, transfected with either myc-FBXO7 or FLAG-LC2 together with appropriate control vectors as well as FLAG-LC2 together with myc-FBXO7 WT along with one of the indicated HA-Ubiquitin variant plasmids were subjected to IB using the FLAG, myc or 14-3-3 antibodies, respectively. The latter served as a loading control. The bracket indicates smear. (f) $75 \mu \mathrm{g}$ of lysates from HEK293T cells, transfected with myc-FBXO7 and FLAG-LC1 were incubated with or without addition of $\lambda$ protein phosphatase for $1 \mathrm{~h}$ at $37^{\circ} \mathrm{C}$ and subjected to IB using the FLAG, SP1 or myc antibodies.

\subsection{FBX07 binds to the proteasome via its novel interaction partner PSMA2 (proteasomal subunit alpha 2)}

Another novel interaction protein identified in the screen was the proteasomal subunit alpha 2 (PSMA2) (Table 2.1). To determine whether PSMA2 is a novel interactor of FBXO7, I transfected HEK293T cells with plasmids encoding control vector, myc-FBXO7 and FLAGPSMA2 and subjected them to co-immunoprecipitation analyses. Specific interaction bands in the experimental as compared to the control conditions confirmed the interaction of FBXO7 and PSMA2 (Figure 2.12 a, b).

a

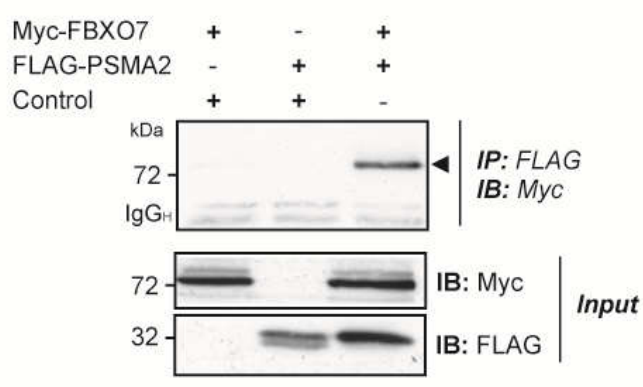

b

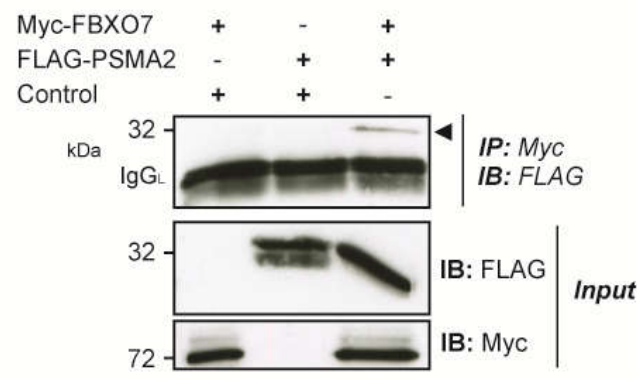

Figure 2.12: FBXO7 interacts with PSMA2: (a) Lysates from HEK293T cells, transfected with either mycFBXO7 or FLAG-PSMA2 together with appropriate respective control vectors or both myc-FBXO7 and FLAGPSMA2 plasmids were subjected to IP with the FLAG antibody and subsequent IB using the myc antibody. 50 $\mu \mathrm{g}$ of total cell lysate was subjected to IB using the same antibodies and served as an input control. Arrowhead indicates specific interaction band. IgGH indicates the heavy chain of the IP antibody. (b) Lysates from HEK293T cells, transfected with either myc-FBXO7 or FLAG-PSMA2 together with appropriate respective control vectors or both myc-FBXO7 and FLAG-PSMA2 plasmids were subjected to IP with the myc 


\subsection{FBXO7 binds to the proteasome via its novel interaction partner PSMA2}

antibody and subsequent IB using the FLAG antibody. $50 \mu \mathrm{g}$ of total cell lysate was subjected to IB using the same antibodies and served as an input control. Arrowhead indicates specific interaction band. IgGL indicates the light chain of the IP antibody.

To investigate, which domain of FBXO7 was responsible for PSMA2-binding, I made use of FBXO7 deletion mutants lacking either of its known protein domains (Figure 2.13 a). Co-immunoprecipitation analysis in HEK293T cells transfected with control vector or either of the myc-FBXO7 deletion mutants along with FLAG-PSMA2 identified the N-terminal ubiquitinlike (Ubl) domain as the interacting domain (Figure $2.13 \mathrm{~b}$ ). Furthermore, I tested whether one of the known patient mutations located at the N-terminus of FBXO7 (T22M) might interfere with its interaction with PSMA2 and found no difference in the quality of the interaction (Figure $2.13 \mathrm{c}$ ).

a

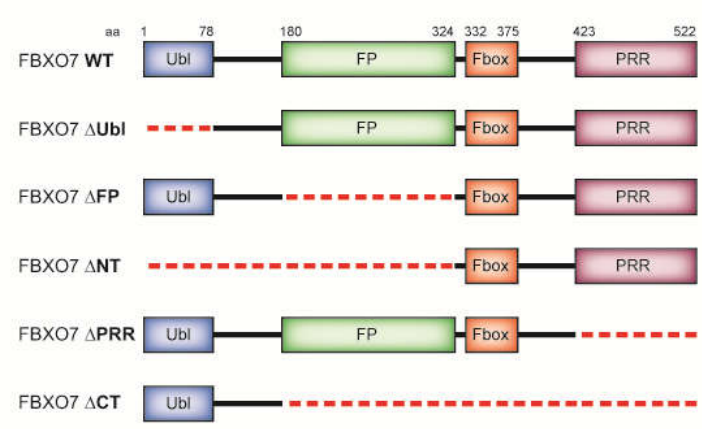

b

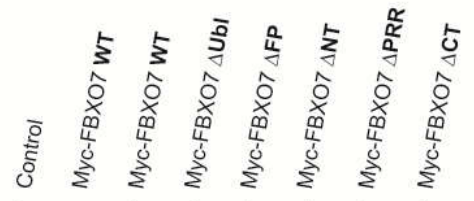

FLAG-PSMA2
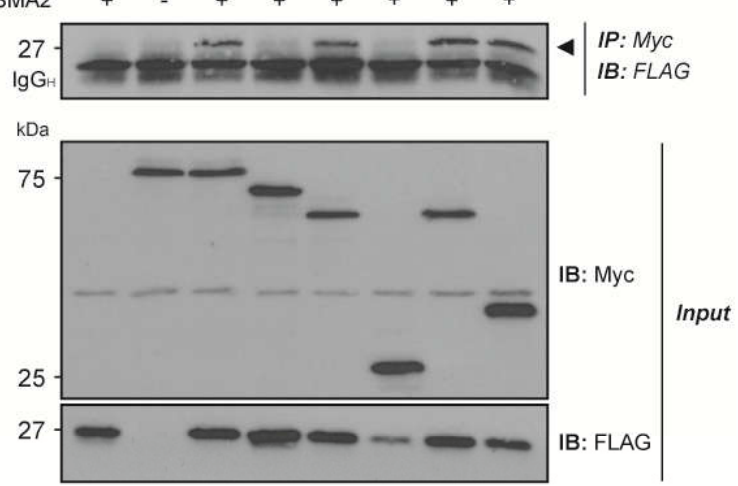

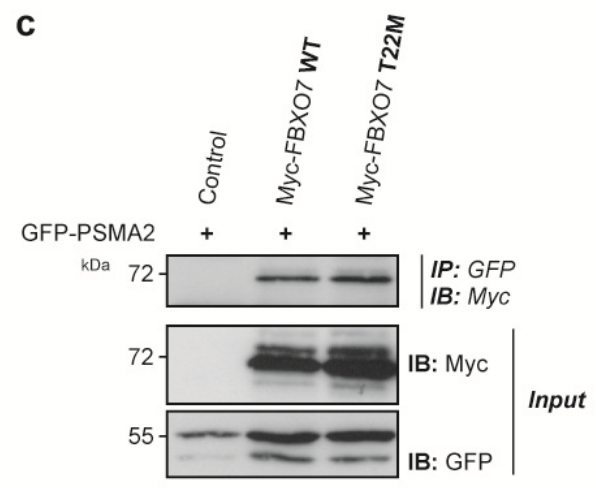

Figure 2.13: FBXO7 interacts with PSMA2 via its Ubl-domain: (a) Schematic representation of $\mathrm{FBXO7}$ deletion ( ) mutants. WT= wild type, Ubl= ubiquitin-like, FP= FBXO7-PI31 interaction domain, NT= Nterminus, $\mathrm{PRR}=$ proline-rich region, $\mathrm{CT}=\mathrm{C}$-terminus. (b) Lysates from HEK293T cells, transfected with either 
2.8 FBXO7 binds to the proteasome via its novel interaction partner PSMA2

FLAG-PSMA2 or myc-FBXO7 with together with appropriate control vectors or FLAG-PSMA2 together with either myc-FBXO7 WT, Ubl, FP, NT, PRR or CT plasmid were subjected to IP with the myc antibody and subsequent IB using the FLAG antibody. $50 \mu \mathrm{g}$ of total cell lysate was subjected to IB using the same antibodies and served as an input control. Arrowhead indicates specific interaction band. IgGL indicates the heavy chain of the IP antibody. (c) Lysates from HEK293T cells, transfected with GFP-PSMA2 together with control, myc-FBXO7 WT or myc-FBXO7-T22M were subjected to IP with the GFP antibody and subsequent IB using the myc antibody. $50 \mu \mathrm{g}$ of total cell lysate was subjected to IB using the same antibodies and served as an input control.

FBXO7 has been shown to interact with the proteasome-interacting protein PI31 (Kirk et al., 2008) and was moreover identified in two mass spectrometry studies designed to identify novel proteasome regulators (Bousquet-Dubouch et al., 2009; Fabre et al., 2015). Although biochemical evidence is still missing, these studies suggest that FBXO7 constitutively binds to the proteasome in a constitutive or transient manner and that FBXO7 may regulate proteasomal processes. To provide biochemical evidence for FBXO7's interaction with the proteasome, I carried out a glycerol gradient density centrifugation. For this, I fractionated transfected HEK293T cell lysates overexpressing either myc-FBXO7 WT, myc-FBXO7 Ubl or myc-FBXO7 FP, the latter two representing loss-of-binding mutants for PSMA2 or PI31, respectively. In the $10-40 \%$ glycerol gradient, the proteasomes sedimented in the bottom fractions. Since proteasomes exist in different assembly forms, which is reflected by the difference in sedimentation coefficients, I observed two peaks of enrichment that represent these forms. Immunoblotting for PSMB5, a subunit of the 20S core, revealed an enrichment between fractions 10 - 12 and fractions 14 - 16 (Figure 2.14 a). Due to the simultaneous presence of the $19 S$ cap protein Rpt6 and the $20 S$ core markers, fractions 14 16 were identified as the 26S / 30S proteasome fractions, whereas fractions 10 - 13 are likely to contain $20 \mathrm{~S}$ particles as well as $20 \mathrm{~S}$ particles associated with alternative regulatory particles. Overexpressed FBXO7 was enriched in the top fractions $3-5$ and is likely to represent cytoplasmic forms of FBXO7. In addition, a substantial amount of FBXO7 cofractionated with the different proteasome forms in fractions $8-13$ and to a lesser degree in fractions 14 - 20 (Figure 2.14 a). Deletion of the PSMA2-interaction domain led to a complete loss of co-fractionation with the proteasome-enriched fractions and an accumulation of the myc-FBXO7 Ubl mutant in fractions 3 - 5 (Figure 2.14 b) suggesting that PSMA2-binding is required for FBXO7's association with the proteasome. In contrast, interaction with PI31 was not required for co-fractionation of $\mathrm{FBXO} 7$ in the proteasome-enriched fractions as deletion of the PI31-interaction domain did not result in a change in the distribution pattern of FBXO7 (Figure 2.14 c). The distribution of myc-FBXO7 FP, however, seemed to be shifted by one fraction towards bottom glycerol concentrations, which can be explained by the reduced size 


\section{Results \\ 2.8 FBXO7 binds to the proteasome via its novel interaction partner PSMA2}

and hence molecular weight of this mutant (Figure 2.14 c, 2.13 a). Taken together, I identified the proteasomal core subunit PSMA2 as a novel interaction partner of FBXO7, mapped the PSMA2-interacting domain to the N-terminal Ubl-domain and provided biochemical evidence that $\mathrm{FBXO} 7$ associates with the proteasome holozenzyme by binding to PSMA2.

a

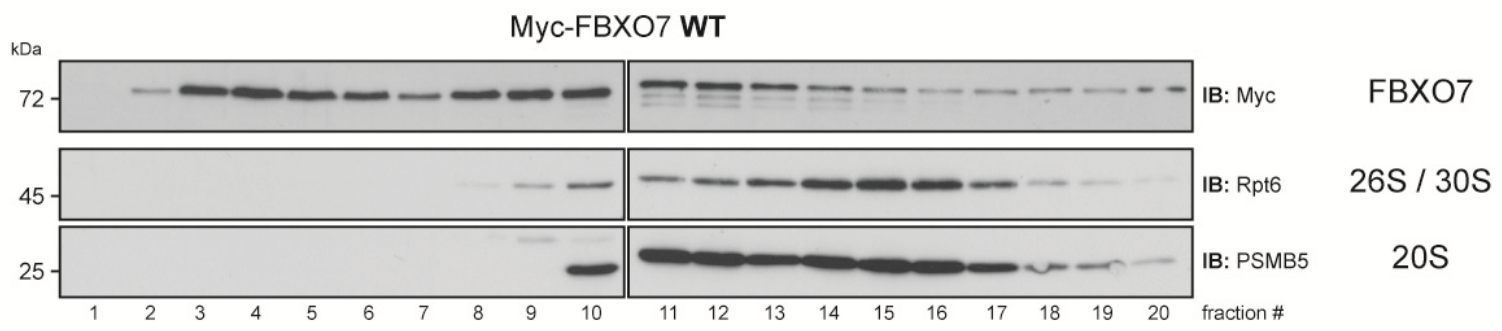

b

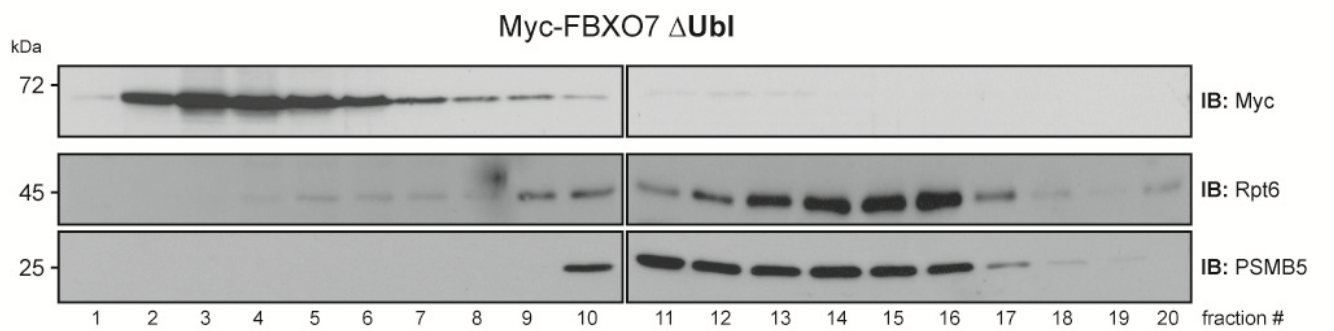

FBXO7

$26 S / 30 S$

$20 S$

C

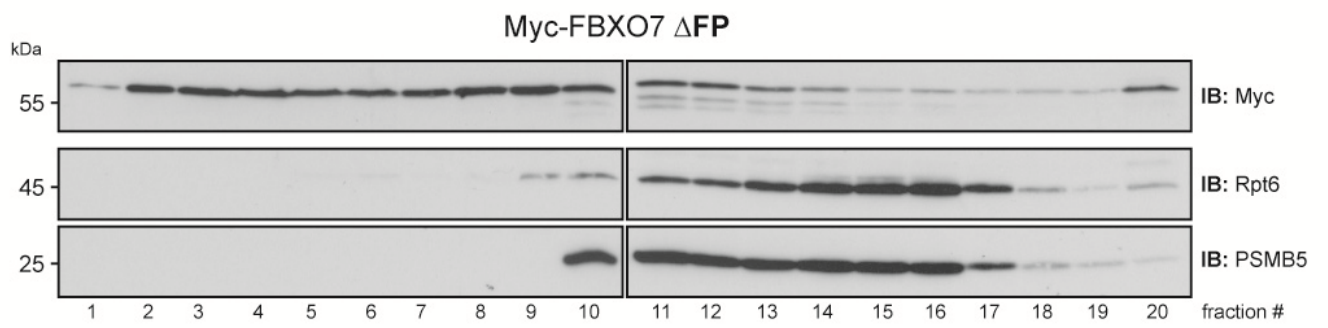

d

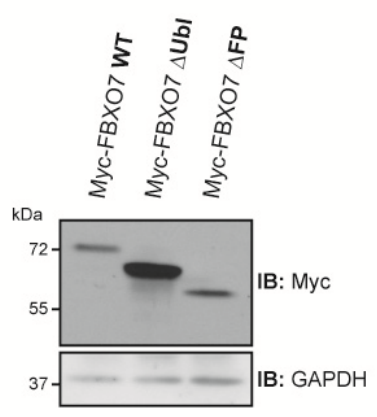

Figure 2.14: FBXO7 associates with the proteasome in a Ubl-domain-dependent manner: (a, b, c) Lysates from HEK293T cells, transfected with either myc-FBXO7 WT (a), myc-FBXO7 Ubl (b) or mycFBXO7 FP (c) were separated by glycerol gradient centrifugation, fractionated and subjected to IB using the FBX07, Rpt6 or PSMB5 antibodies. The latter two served as fraction controls for $26 \mathrm{~S}+30 \mathrm{~S}$ or $20 \mathrm{~S}$ 


\subsection{FBXO7 binds to the proteasome via its novel interaction partner PSMA2}

proteasomes, respectively. (d) Input control for (a, b, c), $50 \mu \mathrm{g}$ of total cell lysate was subjected to IB using the myc and GAPDH antibodies. The latter served as a loading control.

\subsection{FBXO7 ubiquitinates PSMA2 mainly by K63-linked chains with a non-proteolytic outcome}

Having identified PSMA2 as an FBXO7 interaction partner the next question was whether it also is an ubiquitination target. A first hint that this would be the case came from a co-expression analysis in HEK293T cells using control vector, myc-FBXO7 WT or F-box (a mutant lacking the E3 ligase activity-conferring F-box domain) with FLAG-PSMA2. Under these conditions, only co-expression of PSMA2 with FBXO7 WT but not FBXO7 F-box or control vector led to the appearance of a typical ladder-like pattern above the wild type form of PSMA2, indicative of ubiquitination (Figure 2.15 a). Co-expression of another F-box protein FBXO31 did not trigger to this response (Figure 2.15 b) suggesting a FBXO7- and E3 ligase-dependent modification of PSMA2.
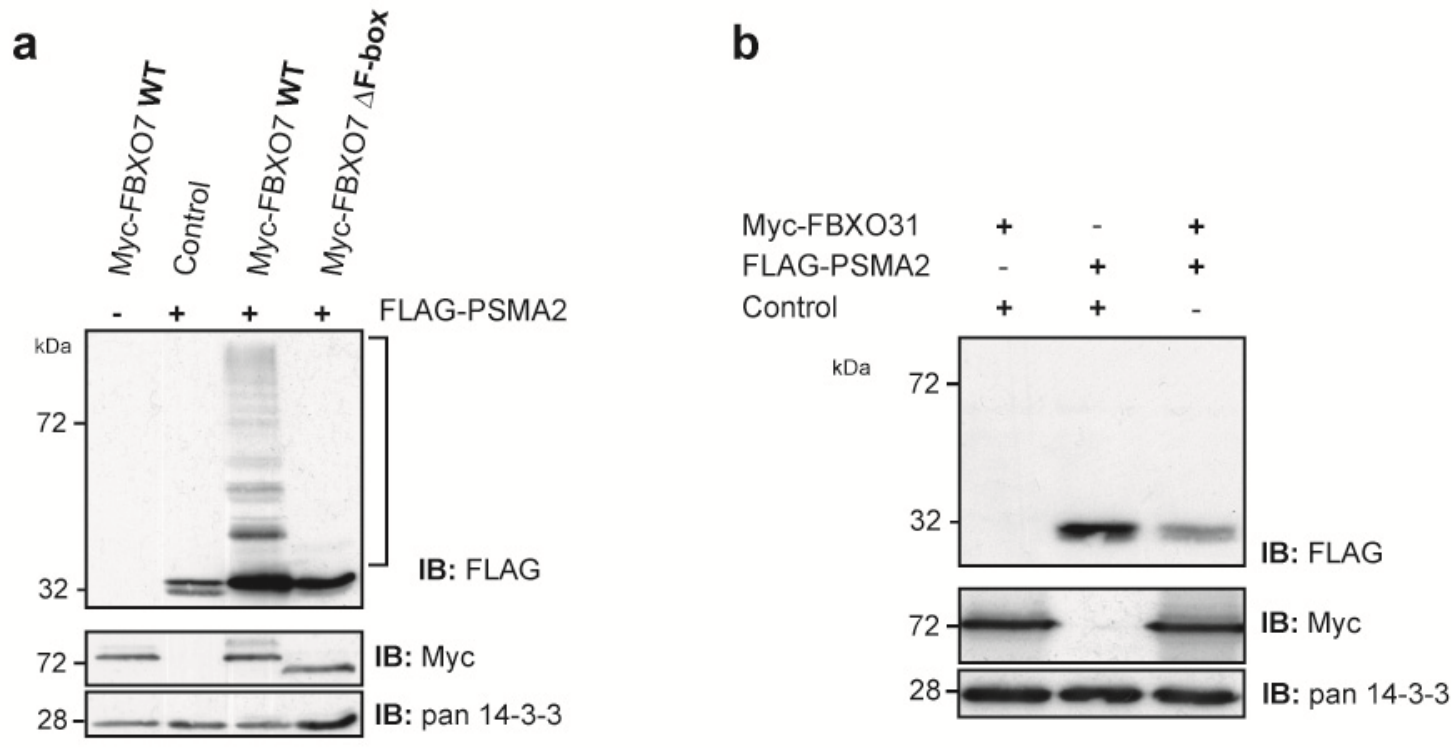

Figure 2.15: PSMA2 is modified in a FBX07-dependent manner: (a) Lysates from HEK293T cells, transfected with either myc-FBXO7 or FLAG-PSMA2 together with appropriate control vectors as well as FLAG-PSMA2 together with either myc-FBXO7 WT or F-box were subjected to IB using the FLAG, myc or 14-3-3 antibodies, respectively. The latter served as a loading control. The bracket indicates smear. (b) Lysates from HEK293T cells, transfected with either myc-FBXO31 or FLAG-PSMA2 with respective control vectors as well as FLAG-PSMA2 together with myc-FBXO31 were subjected to immunoblotting using the FLAG, myc or 14-3-3 antibodies, respectively. The latter served as a loading control. 
To confirm that this was indeed ubiquitination, I carried out a cell-based ubiquitination assay for which I transfected HEK293T cells with control vector, myc-FBXO7 WT or mycFBX07 F-box along with GFP-PSMA2 and subjected them to a lysis protocol under denaturing conditions to avoid contamination of PSMA2 by unspecifically binding proteins. The following immunoprecipitation for GFP-PSMA2 and subsequent immunoblotting with a ubiquitin antibody specific for higher molecular weight ubiquitin chains revealed a ubiquitinpositive smear (Figure 2.16 a). The same experimental conditions were used with a different antibody, which recognizes all forms of ubiquitin conjugates (Figure 2.16 b) confirming that FBXO7 indeed modifies PSMA2 by ubiquitination. Due to the denaturing lysis conditions, all FBXO7 should have ideally been removed from the PSMA2-precipitates. To control for any possible contribution of FBXO7 autoubiquitination to the ubiquitin signal, I carried out a cellbased ubiquitination assay as described before, transfecting only control vector, myc-FBXO7 WT or myc-FBXO7 F-box and immunoprecipitating for myc-FBXO7 followed by immunoblotting with different ubiquitin antibodies. None of the ubiquitin antibodies detected a ubiquitin smear (Figure 2.16 c) suggesting that FBXO7 does not have a high constitutive autoubiquitination activity and furthermore confirming the specificity of ubiquitination to PSMA2 in the previous experiments. 

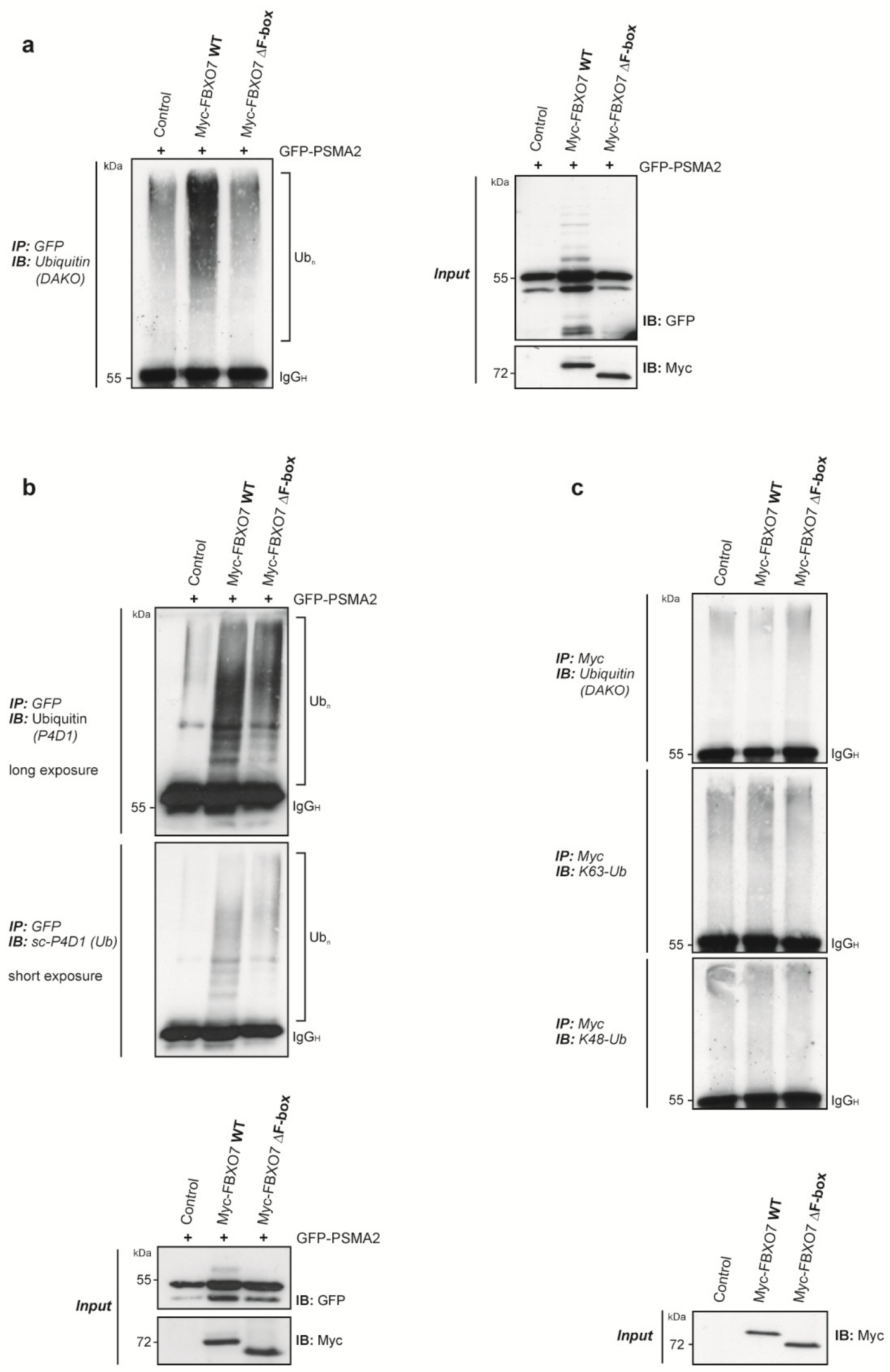
Figure 2.16: FBXO7 ubiquitinates PSMA2: (a) Lysates from HEK293T cells, transfected with GFP-PSMA2 together with either control, FBXO7 WT or FBXO7 F-box were subjected to a denaturing lysis protocol, followed by IP using the GFP antibody. Subsequently, precipitated proteins were immunoblotted using the ubiquitin antibody (DAKO). Ubn indicates the ubiquitin-reactive smear. IgGH indicates the heavy chain of the IP antibody. $50 \mu \mathrm{g}$ of total cell lysate was subjected to IB using the GFP and myc antibodies, serving as an input control. (b) Lysates from HEK293T cells, were transfected and treated as described in (a) followed by IB using the ubiquitin antibody (sc-P4D1). (c) Lysates from HEK293T cells, transfected with either control vector, FBXO7 WT or FBXO7 F-box, subjected to a denaturing lysis protocol and subsequently immunoblotted with the DAKO, K63 polyubiquitin-specfic or K48 polyubiquitin-specific antibodies. IgGH indicates the heavy chain of the IP antibody. $50 \mu \mathrm{g}$ of total cell lysate was subjected to IB using the myc antibody, serving as an input control.

A general concept of gene mutations is interference with the protein function. To test whether any of the reported gene mutations of FBXO7 is interfering with its E3 ligase activity, I subjected HEK293T cells transfected with control vector, myc-FBXO7 WT, the mutants T22M, R378G, R498X and the SNP M115I along with GFP-PSMA2 and tagged HA-Ubiquitin to a denaturing lysis protocol. No ablation of the previously seen ubiquitination smear could be observed (Figure 2.17), suggesting that none of the investigated mutants are interfering with FBXO7's E3 ligase function.

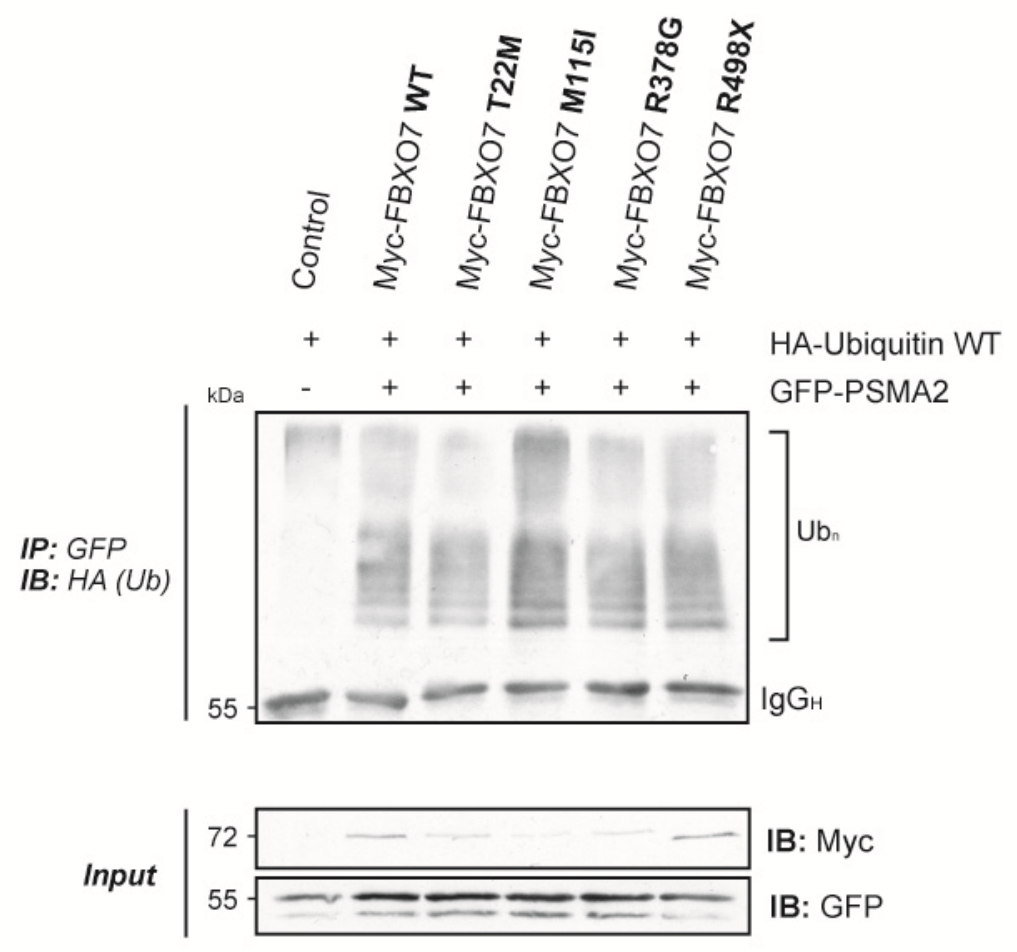

Figure 2.17: FBXO7 mutations do not affect its ligase activity: Lysates from HEK293T cells, transfected with HA-Ubiquitin and GFP-PSMA2 together with either control, FBXO7 WT, T22M, M115I, R378G or R498X were subjected to a denaturing lysis protocol, followed by IP using the GFP antibody. Subsequently, 
precipitated proteins were immunoblotted using the HA antibody. Ubn indicates the ubiquitin-reactive smear. IgGH indicates the heavy chain of the IP antibody. $50 \mu \mathrm{g}$ of total cell lysate was subjected to IB using the GFP and myc antibodies, serving as an input control.

Attachment of ubiquitin to a substrate protein can occur in different modes, either as mono-, multimono-conjugates or polyubiquitin. The latter chains require the specific usage of lysine residues on ubiquitin to assemble polyubiquitin chains. Different types of ubiquitination have a different functional outcome for the protein. The prominent ladder patterns observed in total cell lysates of co-expression of FBXO7 and PSMA2 show distinct bands at molecular weights which correspond to the size of attachment of mono- or multimono- ubiquitin moieties (Figure 2.15 a, 2.16 a - Input panel).

The consistent stability of the ubiquitinated forms of PSMA2 also suggests that the functional outcome of the ubiquitination is non-proteolytic. To examine the type of ubiquitin chain by which PSMA2 is modified (Figure 2.9 b), I subjected HEK293T cells to the same protocol as described, followed by immunoblotting with antibodies specific for either K48- or K63-linked ubiquitin chains. While the K63-specific antibody revealed an increase in smear, the K48-specific antibody did not (Figure 2.18 a). I furthermore took advantage of HAUbiquitin variants that carry mutations in either the lysine residue 48 or 63 and are hence unable to form ubiquitin chains of the respective type. Immunoblotting for the HA antibody revealed that as compared to wild type, mutation of the lysine residue 48 did not lead to a decreased intensity of the smear, while mutation of the lysine 63 residue did (Figure 2.18 b). 
a

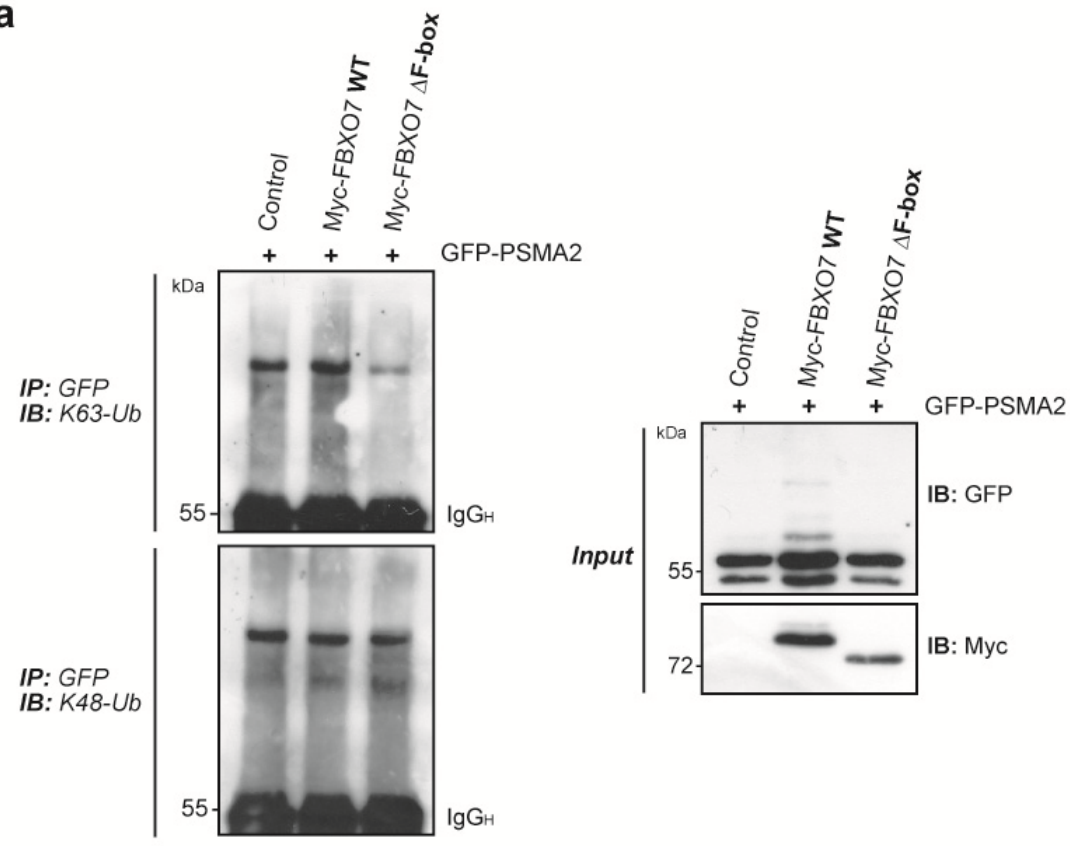

b

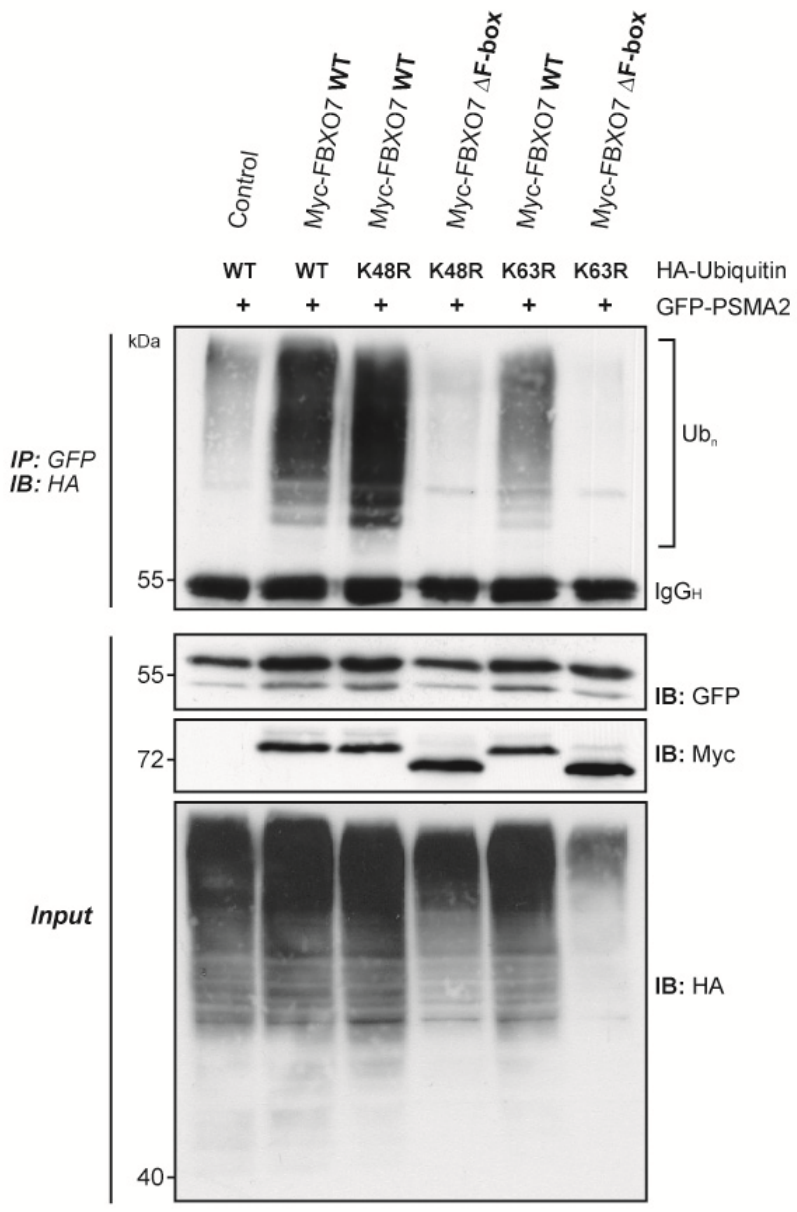


Figure 2.18: FBXO7 ubiquitinates PSMA2 mainly by K63-linked polyubiquitination: (a) Lysates from HEK293T cells, transfected with GFP-PSMA2 together with either control, FBXO7 WT or FBXO7 F-box were subjected to a denaturing lysis protocol, followed by IP using the GFP antibody. Subsequently, precipitated proteins were immunoblotted using the K63 polyubiquitin-specfic or K48 polyubiquitin-specific antibodies. $\mathrm{IgGH}$ indicates the heavy chain of the IP antibody. $50 \mu \mathrm{g}$ of total cell lysate was subjected to IB using the GFP and myc antibodies, serving as an input control. (b) Lysates from HEK293T cells transfected with combinations of plasmids encoding GFP-PSMA2, control, myc FBXO7 WT or F-box and HA-Ubiquitin WT or the indicated linkage-mutant, respectively, were subjected to a denaturing lysis protocol, followed by IP using the GFP antibody. and immunoblotting with the HA antibody. Ubn indicates the ubiquitin-reactive smear. IgGH indicates the heavy chain of the IP antibody. $50 \mu \mathrm{g}$ of total cell lysate was subjected to IB using the GFP, myc and $\mathrm{HA}$ antibodies, serving as an input control.

Collectively, these results suggest that FBXO7 ubiquitinates PSMA2 and that the modification might be mono- or multimono-ubiquitin in addition to lysine 63-linked polyubiquitin chains.

To confirm that the outcome of ubiquitination was indeed non-proteolytic, I compared PSMA2 protein levels in cortical lysates of P18 FBXO7+/+ and FBXO7-/- mice and found no change (Figure 2.19 a, b). Furthermore, PSMA2 protein levels were not notably changed in response to knockdown of FBXO7 in HEK293T cells (Figure 2.22 c), strengthening the idea that the consequence of PSMA2 ubiquitination by FBXO7 is a functional modification rather than degradation.

a

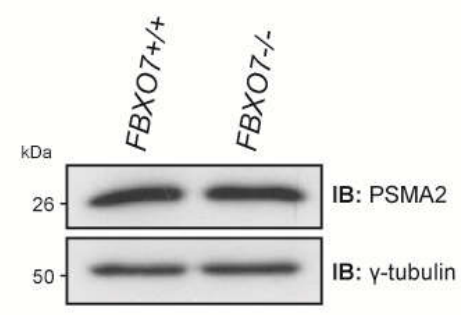

b

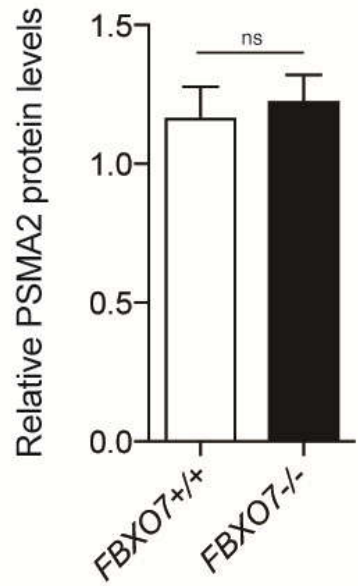

Figure 2.19: PSMA2 stability is not affected in FBXO7-/- animals: (a) P18 cortical lysates of $F B X O 7+/+$ and FBXO7-/- mice were subjected to immunoblotting with the PSMA2 and $y$-tubulin antibodies. The latter served as a loading control. (b) Densitrometric quantification of PSMA2 protein levels in (a). n= $10 \mathrm{FBXO7}$ litterpairs were analyzed (Paired t-test, ns= non significant, mean + s.e.m.). 


\subsection{FBXO7 is required for proper proteasomal assembly and function}

As a subunit of the proteasome core complex, PSMA2 regulates the gating of unfolded protein chains into the proteolytic chamber as well as the assembly of the $20 \mathrm{~S}$ core complex with the $19 S$ regulatory complex (Kish-Trier and Hill, 2013). Functional modification of PSMA2 by FBXO7 would hence be expected to have an impact on either function.

\subsubsection{Proteasome activity is reduced as a result of FBXO7-deficiency}

To first investigate whether proteasome activity in general was affected, I subjected whole brain lysates of $\mathrm{P} 18 \mathrm{FBXO7+/+}$ and FBXO7-/- mice to a proteasome activity assay protocol. Lysates from aforementioned littermates were incubated with the artificial fluorogenic peptide substrate Suc-LLVY-AMC, which is specifically recognized and cleaved by the main protease activity of the proteasome resulting in the release of the fluorophore and the emission of light. As compared to the wild type littermate, brain lysates of FBXO7-/- mice displayed reduced proteasome activity (Figure 2.20 a, b). This result was further corroborated by knockdown of endogenous FBXO7 in the HEK293T heterologous cell system, resulting in a corresponding decrease in proteasome activity (Data Siv Vingill).

a

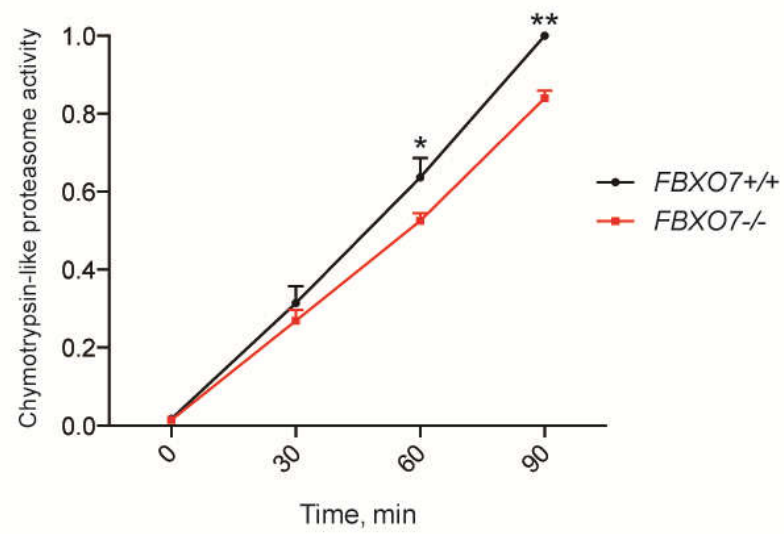

b

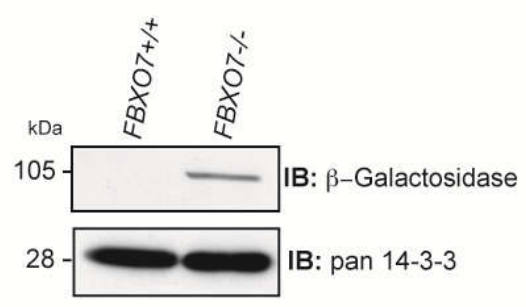

Figure 2.20: Proteasome activity is decreased in the FBXO7-/- brain: (a) P18 whole brains of $F B X O 7+/+$ and FBXO7-/- mice were subjected to a detergent-free lysis protocol. Lysates were incubated with the chymotrypsin-like proteasome activity substrate Suc-LLVY-AMC. Proteasome activity was measured at 0,30 , 60 and $90 \mathrm{~min}$, respectively. Lysates from three independent FBXO7 litters were analyzed and the relative fluorescence normalized to the $\mathrm{FBXO7+/+}$ endpoint quantified (ANOVA, ${ }^{*} \mathrm{p}<0.05$, ${ }^{* *} \mathrm{p}<0.01$, mean + s.e.m.). (b) P18 whole brain lysates from (a) were subjected to immunoblotting using the $\beta$-Gal and 14-3-3 antibodies. The latter served as a loading control. 


\subsection{FBXO7 is required for proper proteasomal assembly and function}

\subsubsection{Loss of FBXO7 does not affect proteasomal gating}

To find out whether the decrease in proteasome activity was a result of less efficient gating of single proteasomes, I subjected cortical lysates of four pooled P18 FBXO7+/+ and FBXO7-/- mice, respectively, to an affinity purification protocol for $26 \mathrm{~S}$ proteasomes. In this protocol, cortical lysates were incubated with the GST-tagged Ubl-domain of RAD23B, which binds 26S proteasomes with high affinity allowing the precipitation of 26S proteasomeRAD23B-Ubl complexes from the lysates. Elution of the $26 \mathrm{~S}$ proteasome complexes from GST-beads was achieved by binding competition for the RAD23B Ubl-domain with the His ${ }_{10^{-}}$ tagged UIM-domain of S5a followed by removal of excess $\mathrm{His}_{10}-\mathrm{UIM}$ with $\mathrm{Ni}^{2+}-\mathrm{NTA}$ beads. The obtained purified 26S proteasome fractions displayed the typical protein band pattern of $26 S$ proteasomes on Coomassie Blue-stained SDS-PAGE gels and had little or no impurities (Figure 2.21 a). Subsequently, $25 \mu \mathrm{g}$ of purified proteasomes from FBXO7+/+ and FBXO7-/brains were resolved by native PAGE and the gel incubated with the artificial substrate SucLLVY-AMC. Both FBXO7+/+ and FBXO7-/- proteasomes displayed comparable activities (Figure 2.21 b, c) suggesting that FBXO7-mediated modification of PSMA2 is not required for proper proteasomal substrate gating or proteasome activity per se. 


\subsection{FBXO7 is required for proper proteasomal assembly and function
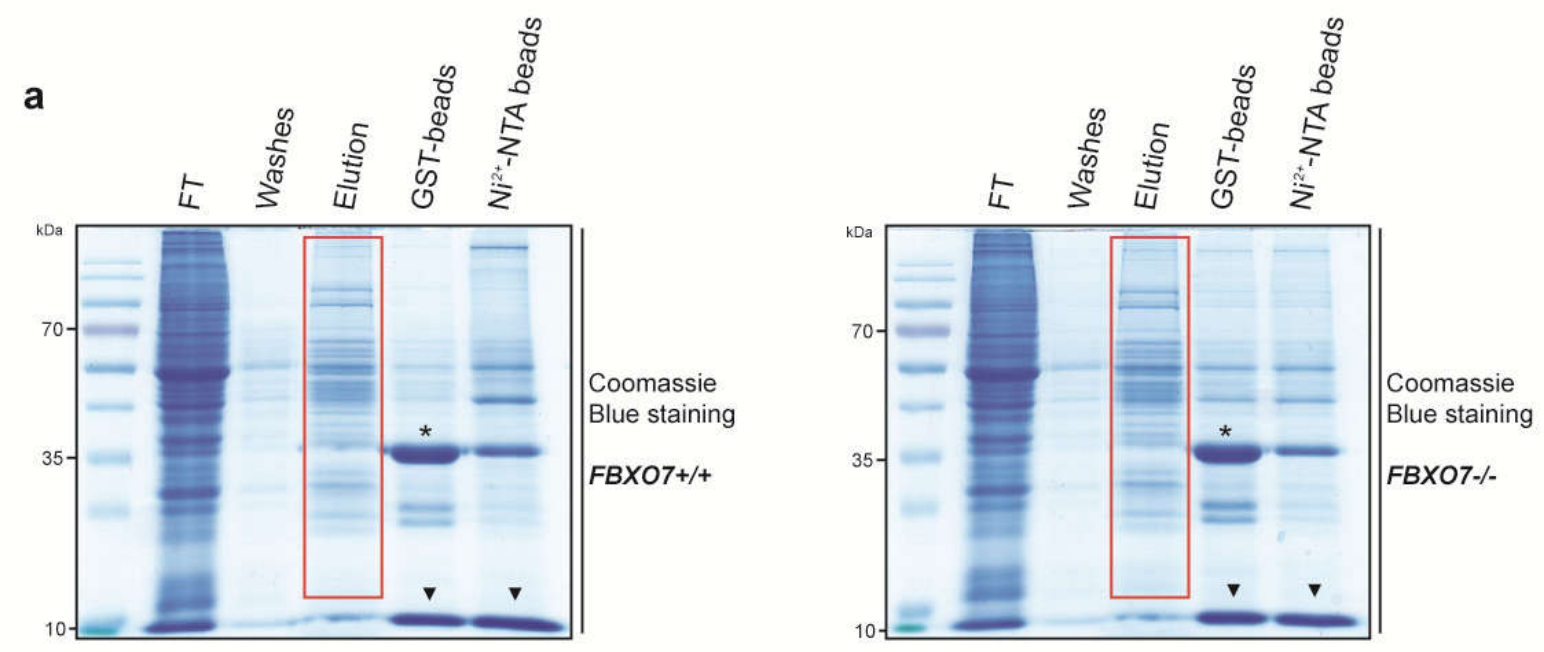

b

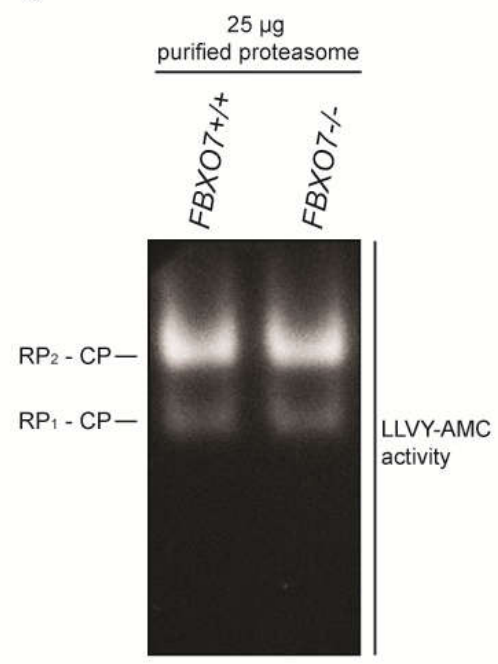

C

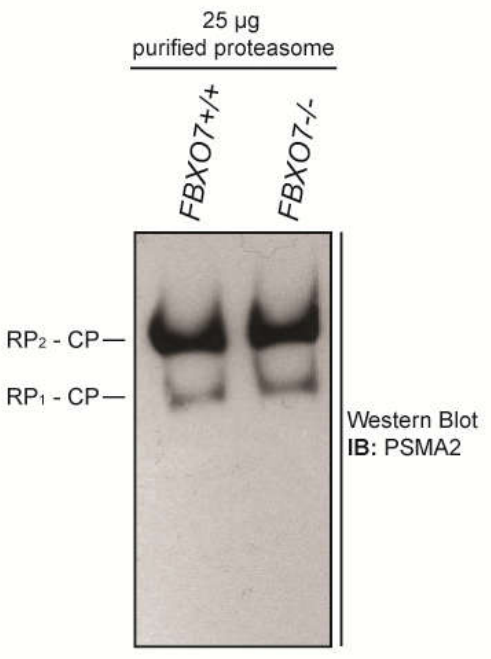

Figure 2.21: Assembled proteasomes from FBXO7-/- mice are functionally active: (a) Pooled cortical lysates of four P18 FBXO7+/+ and FBXO7-/- mice, respectively, were subjected to an affinity purification protocol of $26 \mathrm{~S}$ proteasomes. GST-tagged RAD23B-Ubl was added to the lysates, binding the $26 \mathrm{~S}$ proteasomes and subsequently isolated via GST-beads. Proteasomes were eluted from the beads by

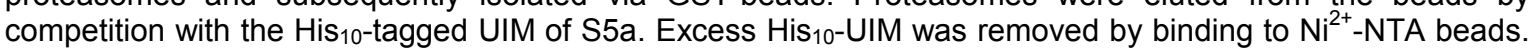
Fractions of each purification step were resolved by SDS-PAGE and stained with the Coomassie blue dye. Asterisk indicates GST-UBL, arrowheads indicate His ${ }_{10}-\mathrm{UIM}$. (b) $25 \mu \mathrm{g}$ of purified proteasomes were resolved by native PAGE and the gel subsequently incubated with Suc-LLVY-AMC for $10 \mathrm{~min}$ at $37^{\circ} \mathrm{C}$. The gel was exposed to UV light and photographed. (c) The same native PAGE gel from (a) was subjected to western blotting and immunoblotted with the PSMA2 antibody. 


\subsection{FBXO7 is required for proper proteasomal assembly and function}

\subsubsection{Loss of FBXO7 causes incomplete assembly of the proteasome holoenzyme}

With proteasome gating remaining functional upon loss-of-FBXO7, the next question was whether proteasome assembly was affected. To test this, I took advantage of a native PAGE gel analysis protocol allowing the visualization of proteasome complexes from cell lysates. HEK293T cell lysates were transfected with empty control vector or RNAi plasmids encoding functional or non-functional FBXO7 shRNA and resolved by native PAGE, followed by incubation of the gel with the artificial substrate Suc-LLVY-AMC. Knockdown of FBXO7 resulted in a marked increase of free $20 \mathrm{~S}$ core particles (CP), which was already visible prior to the stimulation of the latent 20S CP activity with low concentrations of SDS (Figure 2.22 a). While the abundance of single-capped $\left(\mathrm{RP}_{1}-\mathrm{CP}\right)$, so-called $26 \mathrm{~S}$ proteasomes was comparable between knockdown and the controls, the levels of double-capped $\left(\mathrm{RP}_{2}-\mathrm{CP}\right)$, $30 S$ proteasomes was reduced. To confirm, that the differences in the different assembly states of proteasomes reflected changes in abundance of the respective proteasome species and not differences in their activity, the same native PAGE gel was subjected to immunoblotting using the PSMA2 antibody. The results confirmed the overabundance in the FBXO7 RNAi condition (Figure 2.22 b). Analysis of the total cell lysates additionally showed however that total PSMA2 levels were not increased, suggesting that the increase in free $20 S$ core particles was indeed due to assembly deficits of $26 \mathrm{~S}$ or $30 \mathrm{~S}$ proteasomes and not increased production of $20 S$ proteasomes (Figure $2.22 \mathrm{c}$ ). Furthermore, I immunoblotted the same experimental conditions analyzed on native PAGE with the antibody against the proteasome cap subunit Rpt6. This experiment confirmed the decrease in 30S proteasome particles and furthermore revealed an increase in free 19S caps (Figure $2.22 \mathrm{~d}$ ), which was due to assembly deficits and not due to an increase in $19 \mathrm{~S}$ cap production since Rpt6 levels were comparable in total cell lysates (Figure 2.22 e). 


\section{Results

a

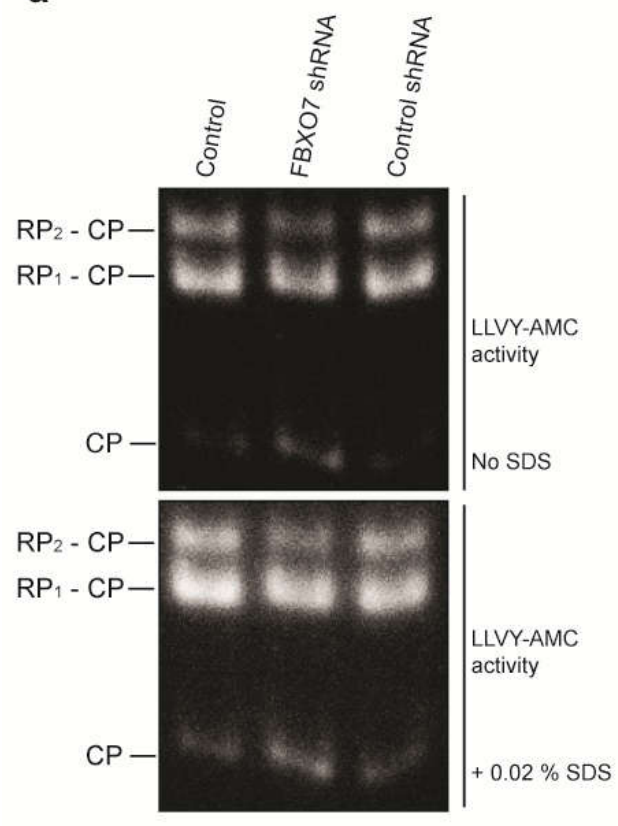

b

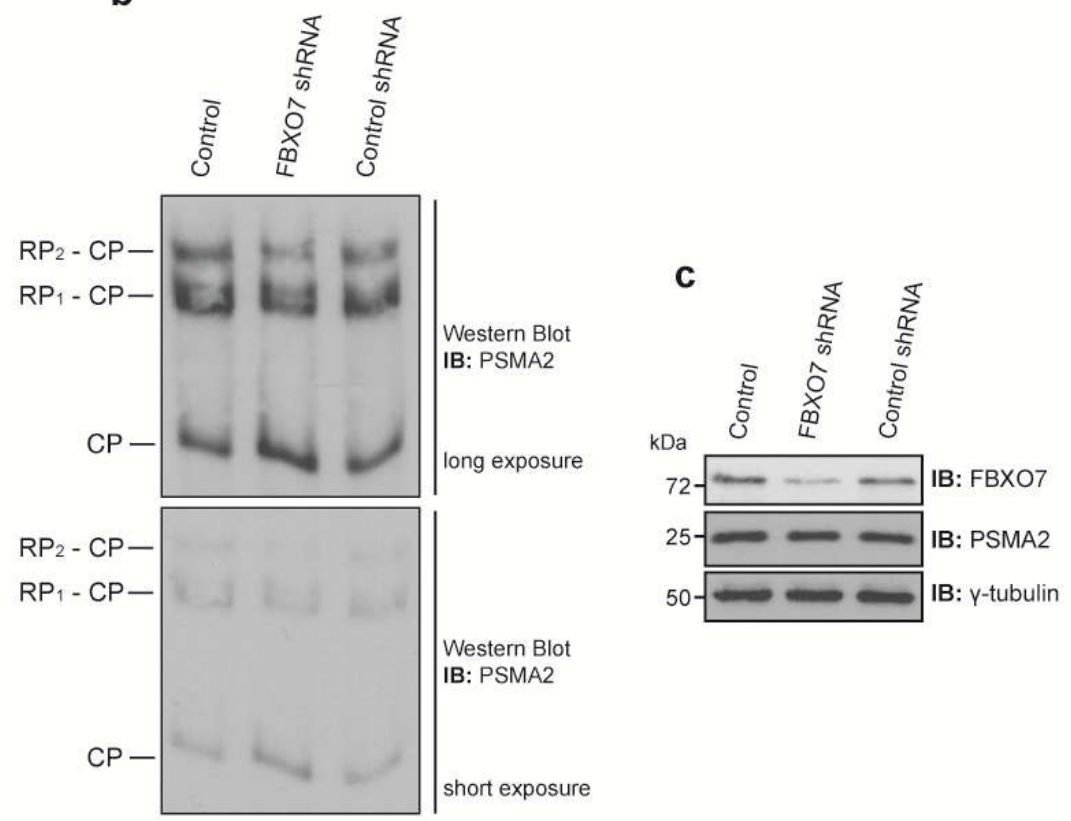

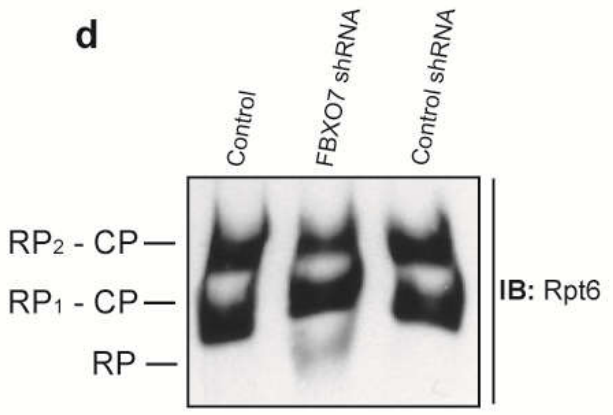

e

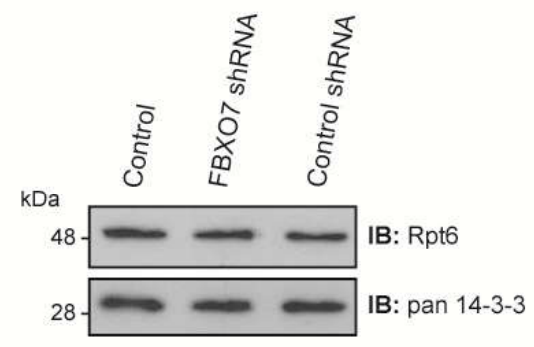

f

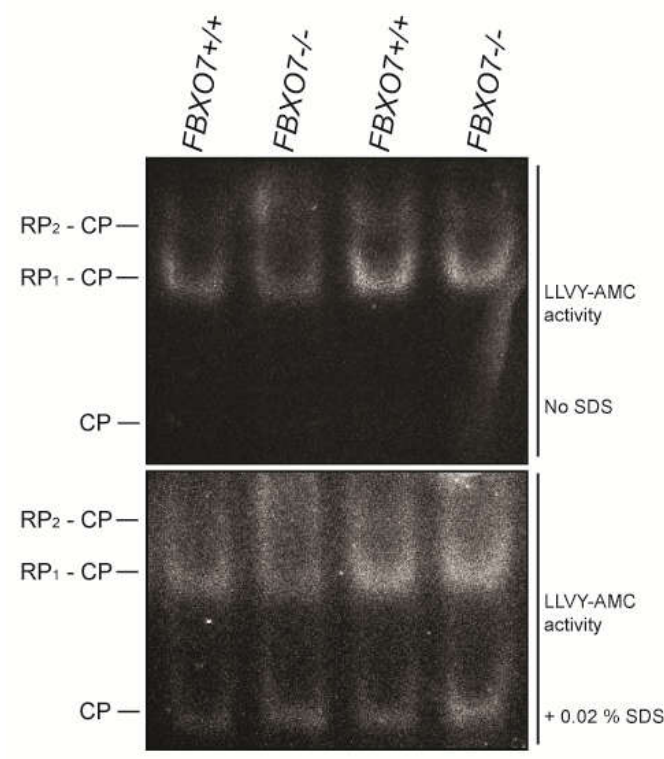

g

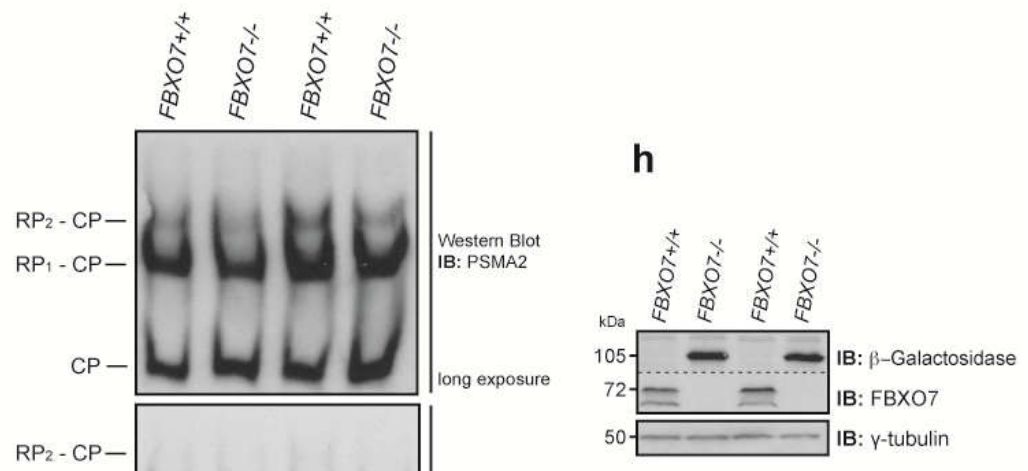




\subsection{FBXO7 is required for proper proteasomal assembly and function}

Figure 2.22: FBXO7 is required for proteasome assembly: (a) Lysates from HEK293T cells, transfected with either control, functional FBXO7 shRNA or non-functional control shRNA were subjected to a detergentfree lysis. Lysates were resolved by native PAGE and the gel subsequently incubated with Suc-LLVY-AMC for $10 \mathrm{~min}$ at $37^{\circ} \mathrm{C}$ in the absence of SDS, followed by $10 \mathrm{~min}$ at $37^{\circ} \mathrm{C}$ in the presence of $0.02 \%$ SDS. After each incubation, the gel was exposed to UV light and photographed. (b) The same native PAGE gel from (a) was subjected to IB with the PSMA2 antibody. (c) Input control for (a) and (b), $50 \mu \mathrm{g}$ of total cell lysate was subjected to IB using the FBX07, PSMA2 and y-tubulin antibodies. The latter served as a loading control. (d) Lysates from HEK293T cells, transfected with either control, functional FBXO7 shRNA or non-functional control shRNA and subjected to a detergent-free lysis protocol. Lysates were resolved by native PAGE and immunoblotted with the Rpt6 antibody. (e) Input control for (d), $50 \mu \mathrm{g}$ of total cell lysate was subjected to IB using the Rpt6 and 14-3-3 antibodies. The latter served as a loading control. (f) Cortices of P18 FBXO7+/+ and FBXO7-/- mice were subjected to a detergent-free lysis protocol. Lysates were resolved by native PAGE and the gel subsequently incubated with Suc-LLVY-AMC for $10 \mathrm{~min}$ at $37^{\circ} \mathrm{C}$ in the absence of SDS, followed by $10 \mathrm{~min}$ at $37^{\circ} \mathrm{C}$ in the presence of $0.02 \%$ SDS. After each incubation, the gel was exposed to UV light and photographed. (g) The native PAGE gel from (f) was subjected to IB with the PSMA2 antibody. (h) $50 \mu g$ of total cell lysate was subjected to IB using the $\beta$-Gal, FBXO7 and $y$-tubulin antibodies. The latter served as a loading control.

Having observed an assembly deficit under short-term conditions of reduced FBXO7 protein levels, I wondered whether this was also true in the FBXO7-/- representing a longterm and complete loss-of-FBXO7. To address this question, I resolved cortical lysates of P18 FBXO7+/+ and FBXO7-/- mice by native PAGE and subjected the gel to incubation with the artificial substrate Suc-LLVY-AMC followed by immunoblotting with the PSMA2 antibody. Both analyzed litterpairs displayed increased levels of free $20 S$ proteasomes in activity and western blot as well as a decrease of 30 s proteasomes in western blot (Figure $2.22 \mathbf{~ f , ~} \mathbf{g}, \mathbf{h}$ ) confirming the results observed under knockdown of FBXO7.

Investigation of the proteasome profile by native PAGE provides a rapid, qualitative tool for profiling of the different assembly states of the proteasome but it lacks a fine, quantitative consideration of the distribution of the proteasome forms as well as their activity levels. To achieve this, I performed glyerol gradient density centrifugation analyses of HEK293T lysates transfected with empty control vector, functional shRNA or non-functional FBXO7 shRNA. As compared to exogenously overexpressed FBXO7 (Figure 2.14 a), endogenous FBXO7 protein was also enriched in fractions 3 - 5 and displayed cofractionation with the proteasome-enriched fractions 8 - 17, however to lower amounts (Figure 2.23 a). Knockdown of FBXO7 expectedly led to a total decrease in FBXO7 protein as well as a decrease in the proteasome fractions (Figure 2.23 b). Measurement of proteasome activity of the control-transfected lysates revealed one major peak of activity in the $26 \mathrm{~S}$ and $30 \mathrm{~S}$ proteasome-enriched fractions 14 - 16 (Figure $2.23 \mathrm{c}$ ). FBXO7 shRNAtransfected cells in contrast displayed a narrower major peak and additionally a smaller peak in the fractions 12 - 14. Measurement of proteasome activity upon SDS treatment, lead to a stimulation of the latent $20 \mathrm{~S}$ proteasome activity and resulted in the expected appearance of 


\subsection{FBXO7 is required for proper proteasomal assembly and function}

an activity peak in the fractions 11 and 12 for the control (Figure $2.23 \mathrm{~d}$ ). In accordance with the data acquired by native PAGE proteasome profiling, knockdown of FBXO7 led to an increase of the stimulated 20 S peak in fractions 11 and 12 as well as a narrower second peak (Figure $2.23 \mathrm{~d}$ ).

Collectively, these results suggest that FBXO7 regulates 19S-20S proteasome assembly, possibly by its direct association with the proteasome at the $20 \mathrm{~S}$ core subunit PSMA2 and its regulation of protein function by ubiquitination. 
a

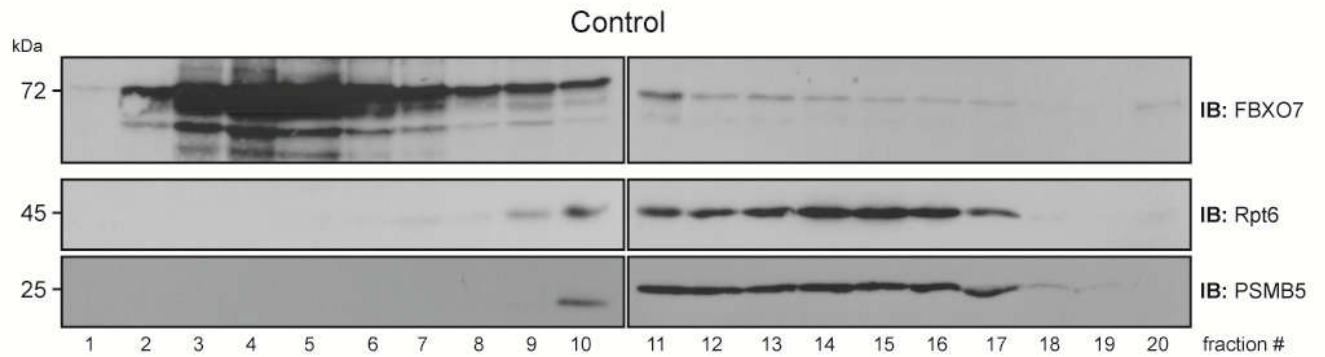

$26 S / 30 S$

$20 S$

b

FBXO7 shRNA

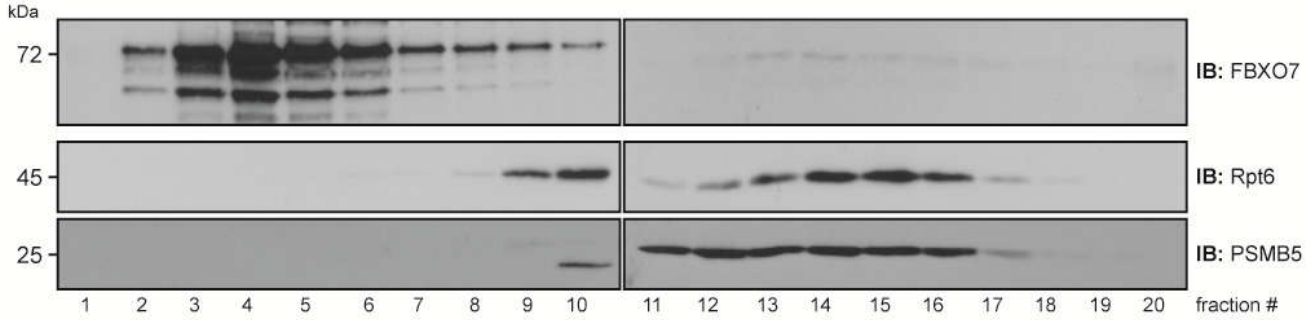

$26 S / 30 S$

$20 S$

C

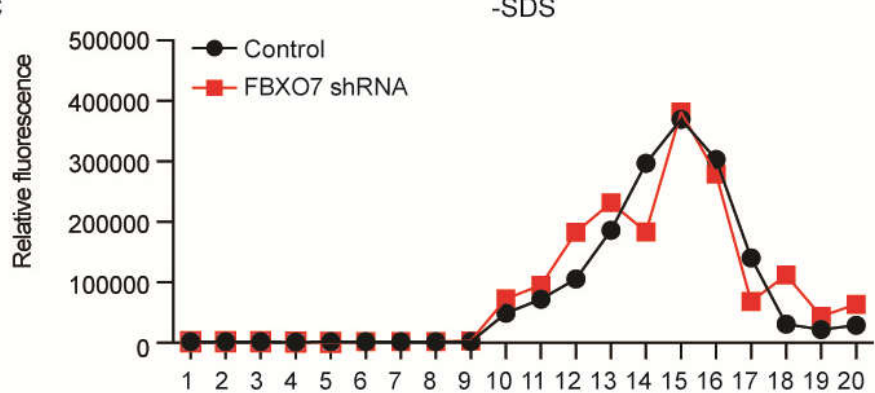

d

+SDS

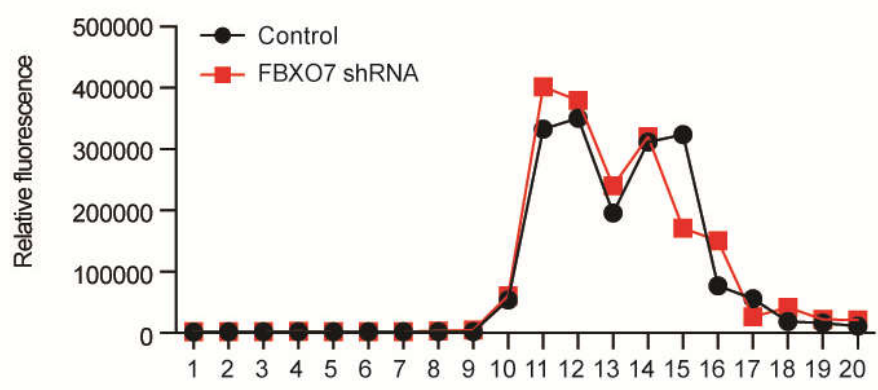

Figure 2.23: Glycerol gradient analysis of FBXO7 knockdown: (a, b) Lysates from HEK293T cells, transfected with either control (a) or FBXO7 shRNA (b) were separated by glycerol gradient centrifugation, fractionated and subjected to IB using the FBXO7, Rpt6 or PSMB5 antibodies. The latter two served as fraction controls for $26 S+30 S$ or $20 S$ proteasomes, respectively. (c, d) Proteasome activity of fractions 1 - 20 was determined by incubation with the chymotrypsin-like proteasome activity substrate Suc-LLVY-AMC either with (d) or without (c) addition of $0.02 \%$ SDS. 


\section{Discussion}

Parkinson's disease is a highly prevalent neurodegenerative disease of unknown cause. In an effort to uncover its etiology, different approaches have been taken. While some lines of research focus on the drug-induced disease models, e.g. the MPTP model, others take genetic and molecular approaches. Familial cases of PD have provided significant starting points by identifying affected genes. Besides the immense fuelling of the alphasynuclein field, this has led to the identification of basic mitochondrial quality control mechanisms, such as the PINK1/parkin pathway. Also the ubiquitin proteasome system (UPS) has been extensively linked to PD but functional insight of UPS components in the disease context is still limited. Mutations in the gene encoding the E3 ligase FBXO7-SCF have recently been identified in familial cases of PD and shown to lead to the disease spectrum PARK15. In this study, I present the effect of loss of the UPS component FBXO7 in mice and shed light onto the so far uncharacterized neuropathology thereof. Furthermore, I provide further insight into the protein function of FBXO7-SCF by identifying a novel ubiquitination substrate. Remarkably, one of the substrates is the alpha 2 subunit of the proteasome core and led me to the finding of proteasomal dysfunction in the FBXO7 knockout brain. My results hence establish the previously anticipated role of FBXO7 in proteasomal regulation and furthermore show that UPS deficiency can be involved in the etiology of PD.

\subsection{Systemic loss of FBXO7 results in detrimental effects on the mouse organism}

A previous study showed that $F B X O 7$ mutations lead to a loss of stability of the main protein isoform 1 in skin fibroblasts isolated from patients (Zhao et al., 2011). This suggests that a loss of protein stability is, at least to a large extent, contributing to the PARK15 loss of function mechanism. To model this, we chose a systemic $F B X O 7$ knockout approach using mice. Loss of $F B X O 7$ had severe effects on general motor behavior and viability. This was in contrast to the mild or absent phenotype in several mouse models of other PARK gene knockout mice (Abeliovich et al., 2000; Gautier et al., 2008; Goldberg et al., 2003) and 


\subsection{Systemic loss of FBXO7 results in detrimental effects on the mouse}

suggests that FBXO7 is a critical component for the entire organism. FBXO7 -/- mice died between P19 and P21 without exception (Data Siv Vingill) and could barely move in the end stage. This is reminiscent of the bedridden state (Di Fonzo et al., 2009; Yalcin-Cakmakli et al., 2014) and premature death (Gunduz et al., 2014; Paisan-Ruiz et al., 2010) of some PARK15 patients. To distinguish the potentially negative impact of organic failure on motor dysfunction, we also generated conditional knockout mouse lines to investigate the contribution of neuronal FBXO7. Deletion of $F B X O 7$ from principal neurons in the forebrain resulted in early-onset motor deficits, whereas deletion of FBXO7 in dopaminergic neurons displayed hypolocomotion, bradykinesia and alterations of the gait, which are also changed in the MPTP model (Data Siv Vingill), (Amende et al., 2005). Taken together, these mouse lines recapitulate the main characteristics of the PARK15 spectrum. The variability of pyramidal tract signs versus parkinsonian symptoms observed in patients is likely due to the varying severity by which the different brain regions are affected in a given genetic background.

Despite the increasing amount of clinical data on PARK15 patients, the underlying neuropathology remains unknown. Since systemic loss of FBXO7 recapitulated the severe phenotype of PARK15 patients, I subjected brain sections of these mice to histopathological examinations. FBXO7-/- mice displayed significantly increased cell death in the cerebral cortex, an effect that I could attribute to the specific loss of FBXO7 by acute knockdown in a primary cortical neuron system. This finding was particularly interesting considering that cortical atrophy was reported in some of the PARK15 patients (Gunduz et al., 2014; PaisanRuiz et al., 2010). Cerebellar and hippocampal regions displayed a tendency to increased cell death, which, however, due to a high variability was not consistent. Furthermore, levels of cell death in the midbrain, number of TH-positive neurons in the midbrain (Data Siv Vingill) and striatal dopamine were unchanged. This suggested that the nigrostriatal system of FBXO7-/mice at P18 was intact. Based on the patients' response to levodopa treatment, an effect on the nigrostriatal system would be expected, however due to the early death of FBXO7-/mice, examination of later degenerative effects was not possible. An absence of nigral neuron degeneration is also observed in other PARK gene knockout mouse models (Goldberg et al., 2005) suggesting that mouse dopaminergic midbrain neurons are more resilient than the human ones. Aside from the cell death in the cerebral cortex, I observed increased levels of astrogliosis. Astrogliosis can accompany neuronal cell death and is also seen in other models, such as the neurotoxic MPTP (Kato et al., 2003) and the genetic ATP13A2 mouse model (Kett et al., 2015). Additionally, I did not observe axonal damage or alpha-synuclein- 
Discussion

3.1 Systemic loss of FBXO7 results in detrimental effects on the mouse organism

positive inclusions. Considering that Lewy bodies are only observed in a few mouse models and moreover not a consistent part of the pathology of early-onset parkinsonism patients (Poulopoulos et al., 2012) this was not surprising. Taken together, these data demonstrate that disruption of the PARK15 gene leads to detrimental consequences for the mouse organism.

\subsection{FBXO7 and the MAP1 protein family - additional regulators of mitochondrial quality control?}

Studies on the protein function of FBXO7 have so far mostly reported ligaseindependent and non-neuronal functions. Thus, identification of novel FBXO7-SCF ubiquitination substrates involved in pathways relevant to the neuronal cell system is crucial. Since ubiquitination substrates have to physically interact with the ligase, I started my analysis by conducting a screen for interaction partners. This screen revealed two novel interactors:

The first interactor was the light chain of microtubule-associated protein 1 B (MAP1BLC1). By carrying out mapping analyses, I found the mitochondria accumulation and genome destruction (MAGD) domain of LC1 to be responsible for FBXO7-binding. This was interesting, since this domain displays a high sequence similarity with MAP1A-LC2 and MAP1S/C19ORF5 (Liu et al., 2005). Subsequent identification of MAP1A-LC2 and MAP1S as additional and novel interactors confirmed that the highly related MAGD domain is indeed responsible for the interaction. While all three MAP1 proteins carry the MAGD domain, its function has only been studied in MAP1S. MAP1S is a cytoplasmic protein that is recruited to stabilized microtubules. Upon accumulation, it causes aggregation of mitochondria leading to cell death and destruction of genomic DNA, for which it uses the eponymous MAGD domain (Liu et al., 2005). In contrast to MAP1S, which is expressed in a variety of tissues (Liu and McKeehan, 2002) MAP1B and MAP1A are only expressed in developing and adult neuronal tissue, respectively (Noiges et al., 2002; Togel et al., 1998). This suggests a critical function of MAP1B-LC1 and MAP1A-LC2 in mitochondrial regulation. MAP1B-LC1 has already been linked to a mitochondrial function via its S-nitrosylation-dependent degradation by the E3 ligase MITOL (Yonashiro et al., 2012). However, the mechanism of action exerted by the MAGD domain remains unknown. The identification of the highly specific binding of FBXO7 to the 25 amino acid MAGD domain suggests that the mitochondrial function of all three MAP1 
family members is carried out in association with FBXO7.My preliminary experiments also suggest, that the FBX07-MAP1 pathway might involve the ubiquitination of these proteins. The recent study by Burchell and colleagues reported a function for FBXO7 in parkin/PINK1dependent mitochondrial regulation (Burchell et al., 2013). FBXO7 was shown to bind to PINK1 and parkin and facilitate parkin-recruitment to defective mitochondria. This raises the question whether FBXO7 binds to mitochondria in a PINK1-dependent manner and which are the triggers for FBXO7 translocation. My data indicates the contribution of a MAP1-FBXO7dependent pathway to the reported mitochondrial quality control function of FBXO7.

\subsection{FBX07 - a novel proteasome regulating protein}

The other identified interactor was the proteasomal subunit alpha 2 (PSMA2). This was particularly interesting because Parkinson's disease and the ubiquitin proteasome system have been linked for a long time but mechanistic insight into UPS dysfunction in this context is still sparse. Moreover, FBXO7 has been shown to interact with PI31 (proteasomal inhibitor 31), a potential proteasome inhibitor (Bader et al., 2011; McCutchen-Maloney et al., 2000), but FBXO7's contribution to proteasome regulation remained unknown.

In this study, I show that FBXO7 binds to PSMA2 via its N-terminal ubiquitin-like (Ubl) domain. This interaction also mediates the association of FBXO7 with the proteasome, as deletion of the Ubl-domain abolished its association with proteasomes. This is consistent with previous studies, which show that Ubl domains are typically present in proteins that associate with the proteasome. The interaction of Ubl domain-containing proteins with the proteasome mostly occurs at the 19S regulatory particle (RP) (Luders et al., 2000; Sakata et al., 2003). FBXO7 however uses the Ubl domain to associate with the $20 \mathrm{~S}$ core particle (CP). Together with two recent mass spectrometry studies (Bousquet-Dubouch et al., 2009; Fabre et al., 2015), my data establishes FBXO7 as a novel proteasome-interacting protein and indicates a proteasome-regulating function. To this end, FBXO7 might act on the proteasome on its own, or as an E3 ubiquitin ligase complex. Proteasomal subunits are known to be posttranslationally modified, including ubiquitination (Cui et al., 2014). Indeed, my data revealed that PSMA2 is a ubiquitination substrate of $\mathrm{FBXO7}$ and that the ubiquitination is dependent on FBXO7's association with the SCF complex. Correspondingly, also SKP1, Cullin1 and Rbx1 have also been detected at proteasomes (Bousquet-Dubouch et al., 2009; Verma et al., 2000). Moreover, I showed that FBXO7-dependent ubiquitination of PSMA2 


\subsection{FBXO7 - a novel proteasome regulating protein}

occurs mainly via K63-linked ubiquitination and that PSMA2 protein levels are unchanged under loss of FBXO7 conditions. This supports the notion that FBXO7 mediates nonproteolytic ubiquitination and likely induces the functional modification of the target protein. Proteasomal alpha subunits have two established functions. The first function is to restrict access to the proteolytic chamber by extension of their C-terminal tails to prevent non-specific proteolysis of proteins. This process is called gating and gate opening is achieved by binding to the RP (Beck et al., 2012; Lander et al., 2012). According to my data however, gating was unaffected as purified proteasomes display unaltered activity in loss of FBXO7 conditions. The second function of the alpha subunits is to interact with subunits of the RP to promote the assembly of proteasome holoenzymes. This process appeared to be affected by loss of FBXO7 as seen in an increase of free CP and RPs, and a decrease in proteasome holoenzymes associated with two RPs. This observation was intriguing given that in contrast to most other alpha subunits, PSMA2 makes additional crucial contact with the RP. All alpha subunits are binding to Rpt1-6 proteins of the base of the RP (Lander et al., 2012; Tian et al., 2011). Due to the asymmetry between the heptameric alpha ring of the core and the hexameric AAA-ATPase base of the RP, however, the contacts appear to be rather weak. An additional, rather surprising direct interaction has been established between the lid of the RP and the CP. Here, the lid subunit Rpn6 directly binds the core subunit PSMA2, stabilizing the weak CP and RP interaction (Pathare et al., 2012). Moreover, this link appeared particularly stable in reconstructions of core particles with two RPs, but not of those with only one RP (da Fonseca and Morris, 2008). This corresponds with my findings that proteasome holoenzymes with two RPs appeared to be more affected compared to holoenzymes with only one. Collectively, my data indicates that the FBXO7-PSMA2 interaction and the functional modification of PSMA2 is essential for maintaining proper proteasome function since free CPs are not capable of ubiquitin-dependent degradation under physiological conditions (Finley, 2009).

\subsection{Conclusion and perspective}

The results from this study establish a role for $\mathrm{FBXO7}$ in regulation of proteasome integrity and activity. The importance of the regulation of proteasome assembly by posttranslational modification has been reported for the subunit PSMA7, which is phosphorylated by protein kinase CK2. PSMA7 phosphorylation appeared to stabilize the association of CP and RPs to form proteasome holoenzymes while loss of phosphorylation in 
response to $\mathrm{Y}$-interferon treatment resulted in unstable holoenzyme complexes (Bose et al., 2004). With PSMA2, this study also uncovers a novel ubiquitination substrate of the FBXO7SCF E3 ligase, which is relevant in the neuronal context. Additionally, a ligase-independent interaction of FBXO7 to PSMA2 could also contribute to the observed effects and it would hence be interesting to differentiate the binding- from ubiquitination-dependent mechanisms.

This study strengthens the link between the UPS and PD by uncovering a mechanism of proteasome regulation that is dysfunctional in the disease context of PARK15. Moreover, FBXO7 seems to be involved in the regulation of two integral cell systems, mitochondria and proteasomes. The reciprocity of these systems makes it difficult to trace which of them would be affected first by FBXO7 deficiency. Loss of FBXO7-mediated mitochondrial dysfunction (Burchell et al., 2013) is known to e.g. lead to an increase in reactive oxygen species (ROS), which has been recently reported to result in proteasomal disassembly (Livnat-Levanon et al., 2014). Conversely, a reduction in proteasome activity has been demonstrated to result in an impairment of mitochondrial homeostasis and turnover. Due to FBXO7's potential to localize to both the proteasome as well as the mitochondria, FBXO7 could represent a protective mechanism of the cell to react to deficiencies in either system. Increased mitochondrial dysfunction, e.g. would be anticipated to result in increased translocation of FBXO7 to mitochondria, which, in turn would lead to reduced FBXO7 on proteasomes. The consequence would be an increase in free core particles, which are thought to be capable of the non-specific degradation of oxidized proteins and hence serve as a preventive mechanism to restrict ROS-mediated damage (Livnat-Levanon et al., 2014). Under conditions with normal mitochondrial function, $\mathrm{FBXO} 7$ would be abundantly available to maintain physiological levels of associated proteasome holoenzymes. In the case of PARK15, leading to a loss of FBXO7, both cell systems are hence affected in their basic functions and would be expected to negatively impact on each other leading to high cellular stress levels. The degree of cell stress in the different cell-types could be modulated by protective compensatory mechanisms inherent to them. Collectively, my findings demonstrate that proteasome function and integrity are dependent on FBXO7 function and emphasize UPS dysfunction as a major contributing mechanism to PD etiology. 


\section{Material and Methods}

\subsection{Materials}

\subsubsection{Chemicals, Reagents and Kits}

Chemicals and reagents used in this study were purchased from Applichem (Darmstadt, Germany), Enzo Life Sciences, Inc. (New York, US), GE Healthcare (New Jersey, US), Invitrogen (Darmstadt, Germany), Merck (Darmstadt, Germany), Roth (Karlsruhe, Germany), Sigma-Aldrich (Munich, Germany), Thermo Fisher Scientific (Massachusetts, US), Th. Geyer (Renningen, Germany), Qiagen (Limburg, Netherlands) or Worthington (New Jersey, US). Cell culture media and supplements were purchased from Gibco $^{\mathrm{TM}}$ / Thermo Fisher Scientific. Commercially available kits include the Nucleobond® and NucleoSpin $®$ kits for DNA isolation and extraction, respectively, from Macherey-Nagel (Dueren, Germany) and the DeadEnd ${ }^{\mathrm{TM}}$ Fluorometric TUNEL System (Promega, US). DNA and protein ladder markers were obtained from Fermentas / Thermo Fisher Scientific.

\subsubsection{Laboratory Equipment}

The following equipment was used in this study: micropipettes (Gilson, USA), consumables and plastic ware (Falcon (Becton Dickinson Labware Europe, Le Pont De Claix, France); Eppendorf (Hamburg, Germany); Greiner Bio-One (Frickenhausen, Germany)), tabletop and ultracentrifuges (Eppendorf (Hamburg, Germany) and Beckmann Coulter (Krefeld, Germany), respectively), rocker and shaker (Heidolph, Germany), thermocycler (Biometra, Germany), heater block (Grant Instruments, UK), UV transluminator (Intas, Germany), spectrophotometer (Amersham Biosciences, UK), Fluorescent plate reader (Wallac 1420 VICTOR $^{2} \mathrm{TM}$ ), fluorescent microscope, inverted light microscope and dissection microscope (Eclipse Ti, Eclipse TS100 and SMZ645, respectively, from Nikon, Japan), brightfield light microscope (Zeiss Axiophot).

\subsubsection{Antibodies}

Primary and secondary antibodies used in this study are listed in Table 4.1 and 4.2. 
Table 4.1: List of primary antibodies.

\begin{tabular}{|c|c|c|c|c|}
\hline Primary antibody & Application & Dilution & Host & Company \\
\hline pan 14-3-3 & WB & $1: 5000$ & $\mathrm{~ms}$ & Santa Cruz \\
\hline$\alpha$-Synuclein & IHC, WB & $1: 250,1: 1000$ & $\mathrm{rb}$ & Cell signaling \\
\hline ß-galactosidase & WB & $1: 500$ & $\mathrm{~ms}$ & Santa Cruz \\
\hline $\mathrm{Y}$-Tubulin & WB & $1: 10000$ & $\mathrm{~ms}$ & Sigma-Aldrich \\
\hline APP & IHC, WB & $1: 250,1: 250$ & $\mathrm{~ms}$ & Thermo Fisher Scientific \\
\hline Cleaved caspase- 3 & ICC & $1: 200$ & $\mathrm{rb}$ & Cell signaling \\
\hline Cullin1 & WB & $1: 500$ & $\mathrm{~ms}$ & Santa Cruz \\
\hline Myc & WB & $1: 500$ & $\mathrm{~ms}$ & Santa Cruz \\
\hline Flag & WB & $1: 1000$ & $\mathrm{~ms}$ & Sigma-Aldrich \\
\hline GFP & WB & $1: 500$ & $\mathrm{~ms}$ & Santa Cruz \\
\hline GFP & ICC & $1: 1000$ & $\mathrm{rb}$ & Thermo Fisher Scientific \\
\hline $\mathrm{HA}$ & WB & $1: 500$ & $\mathrm{~ms}$ & Santa Cruz \\
\hline SP1 & WB & $1: 500$ & $\mathrm{~ms}$ & Santa Cruz \\
\hline GFAP & $\mathrm{IHC}$ & $1: 200$ & $\mathrm{~ms}$ & Nova Castra \\
\hline Iba1 & $\mathrm{IHC}$ & $1: 1000$ & $\mathrm{~ms}$ & Wako \\
\hline Ubiquitin & WB & $1: 500$ & $\mathrm{rb}$ & Dako \\
\hline Ubiquitin (P4D1) & WB & $1: 500$ & $\mathrm{~ms}$ & Santa Cruz \\
\hline FBXO7 & WB & $1: 500-1: 40$ & $\mathrm{~ms}$ & Santa Cruz \\
\hline Ubiquitin K63 & WB & $1: 200$ & $\mathrm{~ms}$ & Millipore \\
\hline Ubiquitin K48 & WB & $1: 200$ & $\mathrm{~ms}$ & Millipore \\
\hline PSMA2 & WB & $1: 1000$ & $\mathrm{rb}$ & Cell signaling \\
\hline PSMB5 & WB & $1: 1000$ & $\mathrm{rb}$ & Cell signaling \\
\hline Rpt6 & WB & $1: 500$ & $\mathrm{~ms}$ & Enzo lifescience \\
\hline GAPDH & WB & $1: 2000$ & $\mathrm{~ms}$ & Abcam \\
\hline
\end{tabular}


Table 4.2: List of secondary antibodies.

\begin{tabular}{c|ccccc|} 
Secondary antibody & Application & Conjugated probe & Dilution & Host & Company \\
\hline anti-mouse IgG & WB & HRP & $1: 10000$ & goat & Dianova \\
anti-rabbit IgG & WB & HRP & $1: 10000$ & goat & Dianova \\
anti-mouse IgG & ICC & Cy2 / Alexa 488 & $1: 1000$ & goat & Dianova \\
anti-rabbit IgG & ICC & Cy3 / Alexa 555 & $1: 1000$ & goat & Dianova \\
\cline { 2 - 6 }
\end{tabular}

\subsubsection{Enzymes}

Enzymes used in this study are listed in Table 4.3.

Table 4.3: List of enzimes.

\begin{tabular}{|c|c|}
\hline Enzyme & Company \\
\hline Restriction Enzymes & New England Biolabs \\
\hline Pfu DNA Polymerase & Fermentas / Thermo Fisher Scientific \\
\hline T4 DNA Ligase & Fermentas / Thermo Fisher Scientific \\
\hline GoTaq® DNA Polymerase & Fermentas / Thermo Fisher Scientific \\
\hline Proteinase $\mathrm{K}$ & AppliChem \\
\hline DNase I & Roche \\
\hline Lambda Protein Phosphatase & New England Biolabs \\
\hline
\end{tabular}

\subsubsection{Buffers and solutions}

10x PBS: $1.37 \mathrm{M} \mathrm{NaCl}, 14.7 \mathrm{mM} \mathrm{KCl}, 78.1 \mathrm{mM} \mathrm{Na}_{2} \mathrm{HPO}_{4}, 26.8 \mathrm{mM} \mathrm{KH}_{2} \mathrm{PO}_{4}$, adjust to $\mathrm{pH} 7.4$

\section{Molecular Biology:}

2x TAE: 80 mM Tris-acetate, 2 mM EDTA, adjust to $\mathrm{pH} 8.5$

2x YT medium (for $1 \mathrm{~L}$ ): $5 \mathrm{~g} \mathrm{NaCl}, 16 \mathrm{~g}$ tryptone, $10 \mathrm{~g}$ yeast extract

2x YT agar plates: add $1.5 \%$ agar and $50 \mu \mathrm{g} / \mathrm{ml}$ ampicillin or kanamycin 


\section{Yeast Two-Hybrid:}

SD (synthetic dropout) media and plates (for $500 \mathrm{ml}$ ): $3.35 \mathrm{~g}$ Yeast nitrogen base w/o amino acids, $X \mathrm{~g}$ Drop out supplement $\{\mathrm{X}=0.69 \mathrm{~g}$-Leu, $0.64 \mathrm{~g}$-Leu/-Trp, $0.72 \mathrm{~g}$-His, $0.62 \mathrm{~g}$-Leu/Trp/-His\} (Clontech), adjust pH to 5.8 and autoclave (for plates: add $10 \mathrm{~g}$ agar). After cooling, add $25 \mathrm{ml}$ of $40 \%$ glucose

2x YPDA (for $250 \mathrm{ml}$ ): $10 \mathrm{~g}$ peptone, $5 \mathrm{~g}$ yeast extract, adjust $\mathrm{pH}$ to 6.5 and autoclave. After cooling, add $25 \mathrm{ml}$ of $40 \%$ glucose

Z-buffer (for $1 \mathrm{~L}$ ): $16.1 \mathrm{~g} \mathrm{Na}_{2} \mathrm{HPO}_{4} \times 7 \mathrm{H}_{2} \mathrm{O}, 5.5 \mathrm{~g} \mathrm{NaH}_{2} \mathrm{PO}_{4} \times \mathrm{H}_{2} \mathrm{O}, 0.75 \mathrm{~g} \mathrm{KCl}, 0.246 \mathrm{~g}$ $\mathrm{MgSO}_{4} \times 7 \mathrm{H}_{2} \mathrm{O}$, adjust $\mathrm{pH}$ to 7 and autoclave

autoclave. After cooling, add $25 \mathrm{ml}$ of $40 \%$ glucose

X-Gal stock: $20 \mathrm{mg} / \mathrm{ml}$ in dimethylformamide

Z-buffer / X-GalX-Gal stock: $10 \mathrm{ml}$ Z-buffer, $167 \mu \mathrm{l}$ X-Gal stock, $27 \mu \mathrm{l}$ ß-

mercaptoethanol

\section{HEK293T cell culture}

HEK293T medium: DMEM [+] 4.5 g/L glucose [-] glutamine [-] pyruvate (Gibco ${ }^{\text {TM }}$ ), 10\% FCS (HyClone), 1\% GlutaMAX® $\left(\right.$ Gibco $\left.^{\mathrm{TM}}\right)$

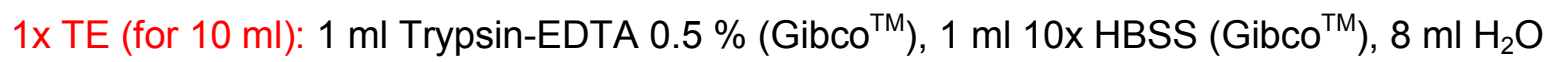

\section{HEK293T and primary neuron culture transfection}

2x HBSS: 50 mM HEPES pH 7.05 - 7.11, 280 mM NaCl, 10 mM KCl, 15 mM glucose, 1.5 mM $\mathrm{Na}_{2} \mathrm{HPO}_{4}$

Nucleofection plating medium: DMEM [+] $4.5 \mathrm{~g} / \mathrm{L}$ glucose [-] L-glutamine [-] pyruvate $\left(\right.$ Gibco $\left.^{\mathrm{TM}}\right), 10 \%$ FCS (HyClone)

\section{Primary cortical neuron cell culture}

HHGN: 1x HBSS (Hank's Balanced Salt Solution), 2.5 mM HEPES pH 7.5, 35 mM glucose $\left(\mathrm{Gibco}^{\mathrm{TM}}\right), 4 \mathrm{mM} \mathrm{NaHCO}_{3}$

TDn: 50 mg Trypsin (Worthington), 5 ml HHGN, $250 \mu \mathrm{l}$ DNase $(2 \mu \mathrm{g} / \mu \mathrm{l})$

Cortical neuron plating medium: Neurobasal ${ }^{\mathrm{TM}}$ [-] L-glutamine $\left(\mathrm{Gibco}^{\mathrm{TM}}\right), 1 \%$ PSG, 2 \% B27 supplement $\left(\mathrm{Gibco}^{\mathrm{TM}}\right)$ 


\section{Immunocytochemistry}

BME / HS: BME [+] Earle's salts [-] L-glutamine $\left(\mathrm{Gibco}^{\mathrm{TM}}\right), 10 \%$ horse serum (heat inactivated)

$8 \%$ PFA: $375 \mathrm{ml} \mathrm{H} \mathrm{O}_{2}$ at $60^{\circ} \mathrm{C}, 50 \mathrm{ml}$ 10x PBS, $40 \mathrm{~g}$ Paraformaldehyde, $40 \mathrm{~g}$ sucrose, adjust to $\mathrm{pH} 7.4$, adjust volume to $500 \mathrm{ml}$ with $\mathrm{H}_{2} \mathrm{O}$

Mounting medium: $12 \mathrm{ml} 0.2 \mathrm{M} \mathrm{pH} 8.5$ Tris- $\mathrm{HCl}, 6 \mathrm{~g}$ glycerol (85\%), $2.4 \mathrm{~g}$ Mowiol 4-88 (Calbiochem), $6 \mathrm{ml} \mathrm{H}{ }_{2} \mathrm{O}, 2.5 \% \mathrm{C}_{6} \mathrm{H}_{12} \mathrm{~N}_{2}$ (DABCO尺)

DAPI: $1 \mu \mathrm{g} / \mu \mathrm{l}$ stock, final concentration 1:8000 in $\mathrm{H}_{2} \mathrm{O}$

\section{Common buffers for biochemistry}

Triton $^{\mathrm{TM}} \mathrm{X}-100$ lysis buffer: $50 \mathrm{mM}$ Tris- $\mathrm{HCl} \mathrm{pH}$ 7.4, $150 \mathrm{mM} \mathrm{NaCl}, 1 \mathrm{mM}$ EDTA, $1 \%$ Triton $^{\mathrm{TM}}$ $\mathrm{X}-100$

Co-IP buffer: 20 mM Tris-HCl pH 7.4, 150 mM NaCl, 1 mM EDTA, $1 \%$ Nonidet $^{\mathrm{TM}}$ P-40, 10\% glycerol

RIPA buffer: $50 \mathrm{mM}$ Tris- $\mathrm{HCl} \mathrm{pH}$ 8.0, $150 \mathrm{mM} \mathrm{NaCl}, 5 \mathrm{mM}$ EDTA, $0.5 \%$ sodium deoxycholate, $1 \%$ Nonidet $^{\mathrm{TM}} \mathrm{P}-40$, w/ or w/o $0.1 \%$ SDS

\section{Subcellular fractionation}

Buffer A: 10 mM HEPES pH 7.9, 10 mM KCl, 0.1 mM EDTA, 0.1 mM EGTA

Buffer $A^{+}$: Buffer A, $0.1 \%$ Nonidet $^{\mathrm{TM}} \mathrm{P}-40$

Buffer C: 20 mM HEPES pH 7.9, 400 mM NaCl, 1 mM EDTA, 1 mM EGTA

\section{Cell-based ubiquitination assay}

UA-Lysis Buffer: $50 \mathrm{mM}$ HEPES pH 7.5, $150 \mathrm{mM} \mathrm{NaCl}, 1.5 \mathrm{mM} \mathrm{MgCl}$, $1 \%$ Triton $^{\mathrm{TM}} \mathrm{X}-100$, $10 \%$ glycerol

HNTG buffer: 20 mM HEPES pH 7.5, 150 mM NaCl, $0.1 \%$ Triton $^{\mathrm{TM}} \mathrm{X}-100,10 \%$ glycerol

\section{Protein purification}

Resuspension / Lysis buffer: 20 mM Tris-HCl pH 7.5, $0.5 \mathrm{M} \mathrm{NaCl}, 0.025 \%$ Nonidet $^{\mathrm{TM}}$ P-40, 1 mM DTT, 20 mM Imidazole

Dialysis buffer: 20 mM Tris pH 7.5, $10 \%$ glycerol, $40 \mathrm{mM} \mathrm{KCl,} 5$ mM MgCl 2,1 mM DTT 


\section{Proteasome affinity purification}

Proteasome lysis buffer C / Purification buffer: 25 mM Tris-HCl pH 7.5, 10 \% glycerol, 5 mM $\mathrm{MgCl}_{2}, 1 \mathrm{mM}$ ATP, 1 mM DTT

\section{Proteasome assays}

Proteasome cell lysis buffer A: $50 \mathrm{mM}$ Tris- $\mathrm{HCl} \mathrm{pH} \mathrm{7.5,} 250 \mathrm{mM}$ sucrose, $5 \mathrm{mM} \mathrm{MgCl}$, $2 \mathrm{mM}$ ATP, $1 \mathrm{mM}$ DTT, $0.5 \mathrm{mM}$ EDTA, $0.025 \%$ digitonin

Proteasome cell lysis buffer $\mathrm{B}: 20 \mathrm{mM}$ Tris-HCl pH 7.4, 5 mM MgCl 2,2 mM ATP, 1 mM EDTA Proteasome activity assay buffer: $50 \mathrm{mM}$ Tris- $\mathrm{HCl} \mathrm{pH} 7.5,40 \mathrm{mM} \mathrm{KCl}, 5 \mathrm{mM} \mathrm{MgCl}, 0.5 \mathrm{mM}$ ATP, $1 \mathrm{mM}$ DTT, $0.05 \mathrm{mg} / \mathrm{ml}$ BSA

Native PAGE activity assay buffer: $50 \mathrm{mM}$ Tris- $\mathrm{HCl} \mathrm{pH} 7.4,5 \mathrm{mM} \mathrm{MgCl}, 1 \mathrm{mM} \mathrm{ATP,} \mathrm{w/} \mathrm{or}$ w/o $0.02 \%$ SDS

Glycerol gradient buffer: 20 mM Tris- $\mathrm{HCl}$ pH 7.4, 5 mM MgCl 2 , 2 mM ATP, 1 mM DTT, 1 mM EDTA, 10 - 40 \% glycerol

\section{SDS-PAGE}

Lower buffer: $1.5 \mathrm{M}$ Tris-HCl pH 8.8, 0.4 \% SDS

Upper buffer: $0.5 \mathrm{M}$ Tris-HCl pH 6.8, 0.4\% SDS

Running buffer: $125 \mathrm{mM}$ Tris, $1.25 \mathrm{M}$ glycine, $0.5 \%$ SDS

Transfer buffer: $20 \mathrm{mM}$ Tris, $153 \mathrm{mM}$ glycine, $20 \%$ methanol

SDS-sample buffer: $300 \mathrm{mM}$ Tris- $\mathrm{HCl} \mathrm{pH}$ 6.8, $50 \%$ glycerol, $10 \%$ SDS, 25\% $\beta$ mercaptoethanol, 0.05\% bromophenol blue

PBST: 1x PBS, $0.1 \%$ Tween-20

\section{Native PAGE}

Native PAGE running buffer: $90 \mathrm{mM}$ Tris base, $90 \mathrm{mM}$ boric acid, $5 \mathrm{mM} \mathrm{MgCl}, 0.5 \mathrm{mM}$ EDTA, $0.5 \mathrm{mM}$ ATP

Native PAGE pre-transfer buffer: $25 \mathrm{mM}$ Tris base, $192 \mathrm{mM}$ glycine, $1 \%$ SDS

Native PAGE sample buffer: 125 mM Tris-HCl pH 6.8, 50 \% glycerol, 2 \% bromophenol blue

\section{Colloidal Coomassie staining}

Dye stock solution: $0.1 \%$ w/v Coomassie Brilliant Blue G-250 (AppliChem), 2 \% w/v orthophosphoric acid, $10 \% \mathrm{w} / \mathrm{v}$ ammonium sulfate

Dye working solution: 80 \% v/v Dye stock solution, $20 \%$ v/v methanol 


\section{Genotyping}

Tail lysis buffer: $10 \mathrm{mM}$ Tris pH 8.0, $200 \mathrm{mM} \mathrm{NaCl}, 10 \mathrm{mM}$ EDTA, $0.5 \%$ SDS

\section{Immunohistochemistry}

X-gal: $1 \mathrm{mg} / \mathrm{ml}$ X-gal in DMSO

X-gal buffer: $2 \mathrm{mM} \mathrm{MgCl}, 0.02 \%$ NP40, $0.01 \%$ sodium deoxycholate, $5 \mathrm{mM}$ potassium ferrocyanide, $5 \mathrm{mM}$ potassium ferricyanide

$0.2 \mathrm{M}$ Phosphate fixation buffer: $0.36 \% \mathrm{w} / \mathrm{v} \mathrm{NaH} \mathrm{NO}_{4}, 3.1 \% \mathrm{w} / \mathrm{v} \mathrm{Na} \mathrm{NPO}_{4}, 1 \% \mathrm{w} / \mathrm{v} \mathrm{NaCl}$

$\mathrm{HCl} /$ Alcohol: $1.25 \mathrm{ml}$ concentrated $\mathrm{HCl}, 500 \mathrm{ml} 70 \% \mathrm{EtOH}$

$4 \%$ PFA for paraffin IHC: $4 \%$ w/v PFA, $0.1 \mathrm{M}$ phosphate fixation buffer

Citrate buffer: $1.8 \mathrm{mM} \mathrm{C}_{6} \mathrm{H}_{8} \mathrm{O}_{7} \times \mathrm{H}_{2} \mathrm{O}, 8.2 \mathrm{mM} \mathrm{C}_{6} \mathrm{H}_{5} \mathrm{Na}_{3} \mathrm{O}_{7} \times 2 \mathrm{H}_{2} \mathrm{O}$, adjust to $\mathrm{pH} 6.0$

Tris buffer: $0.9 \% \mathrm{w} / \mathrm{v} \mathrm{NaCl}, 50 \mathrm{mM}$ Tris- $\mathrm{HCl} \mathrm{pH} 7.6$

BSA/PBS: $0.04 \mathrm{M} \mathrm{NaH}_{2} \mathrm{PO}_{4}, 0.16 \mathrm{M} \mathrm{Na}_{2} \mathrm{HPO}_{4}, 1.8 \% \mathrm{w} / \mathrm{v} \mathrm{NaCl}, 1 \%$ w/v BSA

Blocking buffer: $20 \%$ goat serum v/v in BSA/PBS

\section{Anesthetics}

Ketamine/xylazine: $10 \%$ ketamine v/v (10\% Ketamine, Medistar, Arzneimttelveitrieb $\mathrm{GmbH} 囚), 5 \%$ xylazine (2 \% xylazine, CP Pharma®)

\subsubsection{Plasmid constructs and Primers}

A detailed list of plasmid constructs and primer sequences used in this study is attached as Appendix 1 and Appendix 2.

\subsection{Methods}

\subsubsection{Molecular biology}

\subsubsection{Expression plasmid cloning}

To generate expression plasmids encoding the gene of interest, appropriate primers were designed for subsequent amplification of the cDNA with PCR (Tables 4.4 and 4.5). The annealing temperature of the primers was set $5^{\circ} \mathrm{C}$ below the melting temperature of the respective primer. Elongation time was adjusted to the length gene of interest and the anticipated elongation rate of the Pfu polymerase of $500 \mathrm{bp} / \mathrm{min}$. 
Table 4.4: PCR mix.

\begin{tabular}{|c|c|}
\hline Amount & Reagent \\
\hline $10-50 \mathrm{ng}$ & DNA template \\
\hline $1 \mu \mathrm{l}$ & forward primer $(10 \mathrm{pmol} / \mu \mathrm{l})$ \\
\hline $1 \mu \mathrm{l}$ & reverse primer (10 pmol/ $\mu \mathrm{l})$ \\
\hline $0.4 \mu \mathrm{l}$ & dNTP (25 mM) \\
\hline $0.5 \mu \mathrm{l}$ & Pfu DNA Polymerase (2.5 U/ $\mu \mathrm{l})$ \\
\hline $5 \mu \mathrm{l}$ & 10x Pfu reaction buffer $\left(+\mathrm{MgSO}_{4}\right)$ \\
\hline $41.1 \mu \mathrm{l}$ & $\mathrm{H}_{2} \mathrm{O}$ \\
\hline
\end{tabular}

Table 4.5: PCR program.

\begin{tabular}{c|rcc|}
\hline Program step & Temperature & Time & Repetitions \\
\hline Initial denaturation & $95^{\circ} \mathrm{C}$ & $3 \mathrm{~min}$ & - \\
Denaturation & $95^{\circ} \mathrm{C}$ & $30 \mathrm{sec}$ & \\
Annealing & $\mathrm{X}^{\circ} \mathrm{C}$ & $30 \mathrm{sec}$ & \\
Elongation & $72^{\circ} \mathrm{C}$ & $\mathrm{X} \mathrm{min}$ & \\
Final elongation & $72^{\circ} \mathrm{C}$ & $5 \mathrm{~min}$ & -
\end{tabular}

Following the PCR, amplificates were analyzed using $1 \%$ agarose gel electrophoresis. DNA fragments of expected size were then excised, extracted from the gel using the NucleoSpin ${ }^{\circledR}$ gel clean-up kit and eluted in $20 \mu \mathrm{l} \mathrm{H}_{2} \mathrm{O}$.

Subsequently, the purified PCR product (insert) and $2 \mu \mathrm{g}$ of the target expression vector were digested for $1 \mathrm{~h}$ at $37^{\circ} \mathrm{C}$ using restriction enzymes corresponding to the vector's multiple cloning site. After restriction digest, insert and vector were purified using the NucleoSpin $\circledast$ gel clean-up kit and eluted in $15 \mu \mathrm{l}$ and $50 \mu \mathrm{H}_{2} \mathrm{O}$, respectively. The ligation reaction was set up using $3-5 \mu \mathrm{l}$ of insert combined with $1 \mu \mathrm{l}$ of linearized and dephosphorylated vector and incubated overnight in icy water on the bench (Table 4.6). 
Table 4.6: Ligation mix.

\begin{tabular}{|c|c|}
\hline Amount & Reagent \\
\hline $1 \mu \mathrm{l}$ & Plasmid vector \\
$3.5 \mu \mathrm{l}$ & DNA insert \\
$0.6 \mu \mathrm{l}$ & ATP $(10 \mathrm{mM})$ \\
$1 \mu \mathrm{l}$ & T4 DNA Ligase \\
$1 \mu \mathrm{l}$ & 10x T4 DNA Ligase Buffer \\
$2.9 \mu \mathrm{l}$ & $\mathrm{H}_{2} \mathrm{O}$ \\
\hline
\end{tabular}

After overnight ligation, the full ligation reaction was used to transform $100 \mu \mathrm{l}$ of the chemically competent E.coli strain DH5a. For transformation, bacteria were incubated 30 min on ice with the ligation reaction, followed by a 2 min heat shock at $37^{\circ} \mathrm{C}$ and a $1 \mathrm{~min}$ cool down on ice. Afterwards, $500 \mu$ of $2 x$ YT medium without antibiotics was added and the bacteria incubated for $30 \mathrm{~min}$ at $37^{\circ} \mathrm{C}$. Finally, bacteria were spun down for $5 \mathrm{~min}$ at $4000 \mathrm{rpm}$ and plated onto prewarmed $2 x$ YT agar plates containing the antibiotic matching the vectorencoded antibiotic resistance. Agar plates were incubated overnight at $37^{\circ} \mathrm{C}$. To verify successful ligation of insert and vector, bacterial colonies were first picked and inoculated in 2 $\mathrm{ml} 2 x$ YT medium with antibiotic and incubated overnight at $37^{\circ} \mathrm{C}$ and $175 \mathrm{rpm}$. Then, plasmid DNA extraction from the bacterial culture was carried out using the NucleoSpin $®$ plasmid quick pure kit. Extracted DNA was subjected to a restriction digest to identify positive clones. DNA from putatively positive clones was then submitted for sequencing to the AGCT Lab at the Max Planck Institute of Experimental Medicine, Göttingen.

\subsubsection{Deletion mutant cloning}

For the generation of a gene mutant lacking a given sequence or domain, two sets of primers were designed to carry out fusion PCR. The first set covers the sequence before to the domain to be deleted and includes 15 base pairs of the sequence right after the domain. The second set, in turn, covers 15 base pairs of the sequence before the domain and the sequence after the domain to the stop codon. The so generated PCR product thus has an overlapping stretch to support the annealing of the two fragments. The two sets of primers were then used to run two separate PCRs using $50 \mathrm{ng}$ of template DNA under the previously 
mentioned conditions (Tables 4.4 and 4.5) resulting in the generation of two fragments of the gene, lacking the domain of interest. The partially overlapping fragments were then used as template DNA for a fusion PCR, using the start and the stop codon primers thereby generating a full-length version of the gene lacking the domain of interest. Generation of an expression plasmid containing the deletion mutant was then carried out as described above.

\subsubsection{Yeast Two-Hybrid library screening}

The yeast two-hybrid system was used to screen a cDNA library for potential interaction proteins of FBXO7. The method is based on the fusion of the Gal4 DNA-binding domain (Gal4-BD) to the bait and the Gal4 DNA-activation domain (Gal4-AD) to the prey protein resulting in the functional assembly of the Gal4 transcription factor only in case of interaction of the bait and the prey protein. Assembly of the Gal4 transcription factor then leads to the transactivation of selection genes, allowing the screening of a large library. The method was carried out according to the Matchmaker® Gold Yeast Two-Hybrid System User Manual (Clontech, US).

Full-length FBXO7 was used as bait and cloned into the pGBT9-BD vector carrying the TRP1 nutritional marker. The tryptophane-auxotrophic yeast strain AH109 was used for bait construct transformation. As a first step, the bait construct and the AH109 yeast strain were tested for toxicity and autoactivation. To test for toxicity of the bait construct, AH109 yeast was transformed with the pGBT9-BD-FBXO7 construct followed by plating on SD/-Trp and assessment of colony growth after 2 - 3 days incubation at $30^{\circ} \mathrm{C}$. Autoactivation was assessed by transformation and growth on SD/-His plates.

As prey, the Mate \& Plate $^{\mathrm{TM}}$ Library containing a human fetal brain cDNA library (Clontech, Cat. No. 630469) transformed into the leucine-auxotrophic yeast strain Y187 was used. To perform a control mating, one colony of each yeast strain grown on their respective selection plate was placed in a $1.5 \mathrm{ml}$ reaction tube containing $2 x$ YPDA and vortexed. Subsequently, the mixture was incubated for $24 \mathrm{~h}$ at $30^{\circ} \mathrm{C}$ and $200 \mathrm{rpm}$. The mated culture was then plated on $S D /-T r p /-L e u$ plates and incubated at $30^{\circ} \mathrm{C}$ for $3-5$ days

For the main culture of mating, a fresh bait strain colony was inoculated in $50 \mathrm{ml} S D /-$ Trp medium and incubated at $30^{\circ} \mathrm{C}$ and $250 \mathrm{rpm}$ until $\mathrm{OD}_{600}=0.8(13 \mathrm{~h})$. Cells were then pelleted by centrifugation for $5 \mathrm{~min}$ at $1000 \mathrm{~g}$ and adjusted to a cell density of $>1 \times 10^{8}$ cells / $\mathrm{ml}$ in a total of $5 \mathrm{ml} \mathrm{SD/-Trp} \mathrm{medium.} \mathrm{Next,} \mathrm{a} 1 \mathrm{ml}$ aliquot of the library strain was thawed out, $10 \mu \mathrm{l}$ removed and plated on SD/-Leu plates for library titer determination, and the rest used 
for mating. For mating, $1 \mathrm{ml}$ of the Mate \& Plate library was combined with $5 \mathrm{ml}$ bait strain in a $2 \mathrm{~L}$ flask containing $45 \mathrm{ml}$ of $2 x$ YPDA supplemented with $50 \mu \mathrm{g} / \mathrm{ml}$ kanamycin. The mating culture was then incubated for $24 \mathrm{~h}$ at $30^{\circ} \mathrm{C}$ and $50 \mathrm{rpm}$. Subsequently, cells were centrifuged for $10 \mathrm{~min}$ at $1000 \mathrm{~g}$ and resuspended with $100 \mathrm{ml}$ of $0.5 x$ YPDA supplemented with $50 \mu \mathrm{g} / \mathrm{ml}$ kanamycin. Following another centrifugation for $10 \mathrm{~min}$ at $1000 \mathrm{~g}$, cells were resuspended in $10 \mathrm{ml}$ of $0.5 x$ YPDA/Kan and $100 \mu \mathrm{l}$ of the mated culture plated on SD/-Trp, SD/-Leu and $S D /-T r p /-L e u$ plates to determine the mating efficiency. The remainder of the culture was then spread with $200 \mu \mathrm{l}$ per $150 \mathrm{~mm}$ SD/-Trp/-Leu/-His plates and incubated at $30^{\circ} \mathrm{C}$ for 5 days. Specific colonies (>2 mm) were then picked and replica-plated on prewarmed SD/-Trp/-Leu/His plates.

To eliminate false-positive clones, a ß-galactosidase assay was performed using one of the replica plate sets. For this, a nitrocellulose membrane was placed on the replica plate for $1 \mathrm{~min}$ and then dipped into liquid nitrogen for $30 \mathrm{sec}$. After $20 \mathrm{sec}$ of thawing, the membrane was placed onto a Whatman paper soaked in Z-buffer / X-Gal solution and incubated for $30 \mathrm{~min}-3 \mathrm{~h}$ at RT followed by identification of the ß-gal-producing clones.

ß-gal-positive clones were then subjected to colony PCR (Tables 4.7 and 4.8) and submitted for sequencing to the AGCT Lab at the Max Planck Institute of Experimental Medicine, Göttingen.

Table 4.7: Colony PCR mix.

\begin{tabular}{c|c|} 
Amount & Reagent \\
\hline $1 \mu \mathrm{l}$ & mix with pipette tip dipped in the colony \\
$1 \mu \mathrm{l}$ & reverse primer $1681(10 \mathrm{pmol} / \mu \mathrm{l})$ \\
$2.1 \mu \mathrm{l}$ & $\mathrm{dNTP}(2.5 \mathrm{mM})$ \\
$0.1 \mu \mathrm{l}$ & GoTaq $\AA$ DNA Polymerase \\
$5 \mu \mathrm{l}$ & $5 \times$ GoTaq $囚$ reaction buffer \\
$10.8 \mu \mathrm{l}$ & $\mathrm{H}_{2} \mathrm{O}$
\end{tabular}


Table 4.8: Colony PCR program.

\begin{tabular}{c|rcc|}
\hline Program step & Temperature & Time & Repetitions \\
\hline Initial denaturation & $95^{\circ} \mathrm{C}$ & $3 \mathrm{~min}$ & - \\
Denaturation & $95^{\circ} \mathrm{C}$ & $30 \mathrm{sec}$ & \\
Annealing & $X^{\circ} \mathrm{C}$ & $30 \mathrm{sec}$ & \\
Elongation & $72^{\circ} \mathrm{C}$ & $5 \mathrm{~min}$ & \\
Final elongation & $72^{\circ} \mathrm{C}$ & $10 \mathrm{~min}$ & - \\
\hline
\end{tabular}

\subsubsection{Cell culture and transfection}

Experimental work with cell cultures was carried out in a biological safety cabinet (HERAsafe $\circledast$, Thermo Fisher Scientific). Cultured cells and cell lines were maintained in an incubator (HERAsafe $\AA$, Thermo Fisher Scientific) at $37^{\circ} \mathrm{C}$ with $5 \% \mathrm{CO}_{2}$.

\subsubsection{HEK293T cell culture}

HEK293T cells were maintained in HEK293T medium on $10 \mathrm{~cm}$ culture dishes (Falcon®, Corning). Regular passaging was achieved by cell splitting at a confluency of $90 \%$ by briefly rinsing the cells with $3 \mathrm{ml}$ PBS, followed by incubation with $2 \mathrm{ml} 1 x$ TE for $5 \mathrm{~min}$ at $37^{\circ} \mathrm{C}$. Stopping of the trypsin digest and detachment of the cells from the cell culture dish was carried out by suspending the cells in $8 \mathrm{ml}$ HEK293T medium. Subsequently, cells were collected and centrifuged for $5 \mathrm{~min}$ at $4^{\circ} \mathrm{C}$ and $800 \mathrm{rpm}$. The supernatant was then discarded and the cell pellet resuspended in fresh HEK293T medium. Resuspended cells were plated onto either $10 \mathrm{~cm}$ culture dishes at a dilution of $0.5-2 \mathrm{ml}$ cell suspension in $8 \mathrm{ml} \mathrm{HEK293T}$ medium or onto 6-well plates (CELLSTAR®, Greiner Bio-One) at a dilution of $200 \mu \mathrm{l}$ cell suspension in $2 \mathrm{ml}$ HEK293T medium.

\subsubsection{Transfection of HEK293T cells}

HEK293T cell transfection was carried out using an adapted version of the calcium phosphate method described in (Konishi et al., 2004). 
Cells were split onto 6-well plates the day before and transfected once the cells reached a confluency of $80 \%$. For each 6-well, 0.01 - $3 \mu \mathrm{g}$ of plasmid DNA was diluted in 90 $\mu \mathrm{l}$ of $\mathrm{H}_{2} \mathrm{O}$, followed by addition of $10 \mu \mathrm{l}$ of $2.5 \mathrm{M} \mathrm{CaCl}_{2}$ and $100 \mu \mathrm{l}$ of $2 x \mathrm{HBSS}$. Subsequently, the DNA calcium phosphate solution was pipetted up and down to ensure proper mixing and incubated $5 \mathrm{~min}$ at RT. Finally, the entire DNA mix was added to the 6-well and incubated with the cells 48 and $96 \mathrm{~h}$ prior to lysis for overexpression and knockdown plasmids, respectively.

\subsubsection{Primary cortical neuron culture}

Cortices from P0 Wistar rats were isolated in 1x HBSS (Hank's Balanced Salt Solution) and separated from meninges as well as the hippocampus. Following three washes with $1 x$ HBSS, cortices were incubated with $5 \mathrm{ml}$ of TDn for $10 \mathrm{~min}$ at $37^{\circ} \mathrm{C}$. To stop the trypsin digest, cortices were washed three times with 1x HBSS and subsequently triturated in $5 \mathrm{ml}$ of cortical neuron plating medium with $250 \mu \mathrm{l}$ of freshly added DNase $(2 \mathrm{mg} / \mathrm{ml})$. The cell suspension was then centrifuged for $5 \mathrm{~min}$ at $4^{\circ} \mathrm{C}$ and $800 \mathrm{rpm}$ and the pellet resuspended in $20 \mathrm{ml}$ of cortical neuron plating medium. Plates and $12 \mathrm{~mm}$ coverslips were coated with polyornithine for at least $30 \mathrm{~min}$ at $37^{\circ} \mathrm{C}$ followed by two washes with $\mathrm{H}_{2} \mathrm{O}$ and drying at $37^{\circ} \mathrm{C}$. Cell titer was determined using a hemocytometer. $0.2-0.3 \times 10^{6}$ cells were plated per 24-well and 6 - $10 \times 10^{6}$ per 6-well.

\subsubsection{Transfection of primary cortical neurons}

Primary cortical neurons grown on $12 \mathrm{~mm}$ coverslips in a 24-well plate were transfected at day in vitro (DIV) 1 for overexpression analysis and at DIV3 for survival assays using a modified version of the previously described calcium phosphate transfection method. First, the conditioned culture medium was collected, stored at $37^{\circ} \mathrm{C}$ and replaced with prewarmed 1x DMEM ( [+] $4.5 \mathrm{~g} / \mathrm{L}$ glucose [-] L-glutamine [-] pyruvate (Gibco $\left.{ }^{\mathrm{TM}}\right)$ ). After one wash with 1x DMEM, neurons were starved in $500 \mu \mathrm{l} 1 \times \mathrm{x}$ DEM for $30-45 \mathrm{~min}$ at $37^{\circ} \mathrm{C}$. In the meanwhile, 0.02 - $3 \mu \mathrm{g}$ of plasmid DNA was diluted in $18 \mu \mathrm{l}$ of $\mathrm{H}_{2} \mathrm{O}$, followed by addition of 2 $\mu \mathrm{l}$ of $2.5 \mathrm{M} \mathrm{CaCl}_{2}$ and $20 \mu \mathrm{l}$ of $2 x$ HBSS. Subsequently, the DNA calcium phosphate solution was pipetted up and down to ensure proper mixing and incubated $5 \mathrm{~min}$ at RT. The entire DNA mix was then added to the 24-well and incubated with the neurons for $14-18 \mathrm{~min}$ at $37^{\circ} \mathrm{C}$. Finally, neurons were washed twice with 1x DMEM before the stored conditioned medium was returned. 


\subsubsection{Nucleofection of primary cortical neurons}

Following the primary cortical neuron culture, $8 \times 10^{6}$ rat cortical neurons were resuspended in $100 \mu \mathrm{l}$ of $1 \mathrm{x}$ DMEM and 7 - $10 \mu \mathrm{g}$ plasmid DNA added. The DNA-cell suspension was then subjected to electrical pulsing using the 0-005 program of the nucleofector (Amaxa $\circledast$, Lonza) and incubated with $1 \mathrm{ml}$ of prewarmed 1x DMEM for $5 \mathrm{~min}$ at RT. Finally, neurons were plated onto polyornithine-coated 6-well plates with prewarmed nucleofection plating medium. After $4 \mathrm{~h}$, the medium was replaced by cortical neuron plating medium.

\subsubsection{Immunocytochemistry}

Cortical neuron cultures subjected to survival assays were fixed with $8 \%$ PFA, which was directly added to the conditioned medium to a final concentration of $4 \%$ and incubated for $10 \mathrm{~min}$ at RT. Cells for morphological analyses were washed twice with PBS and then fixed with $4 \%$ PFA for 10 min at RT. Following fixation, cells were washed twice with PBS and then permeabilized with $0.04 \% \operatorname{Triton}^{\mathrm{TM}} \mathrm{X}-100$ in PBS for 10 min at RT. After two more washes with PBS, cells were blocked using $B M E$ / HS for 30 min at RT. The primary antibody was diluted and applied in BME / HS and incubated for either $1 \mathrm{~h}$ at RT or overnight at $4^{\circ} \mathrm{C}$. Subsequently, cells were washed twice with $B M E / H S$ and then incubated with the secondary antibody diluted in BME / HS for $30 \mathrm{~min}$ at RT. Following two washes with PBS, the cells were incubated with the DNA dye DAPI (4'6-diamidino-2-phenylindole) for $10 \mathrm{~min}$ at RT, then rinsed twice more with PBS and finally mounted using mounting medium on microscope slides (Marienfeld).

\subsubsection{Survival assay in primary cortical neurons}

The survival assay was carried out using an adapted version of the method described in (Becker and Bonni, 2004). Cortical neurons plated on $12 \mathrm{~mm}$ coverslips were transfected at DIV3 with $0.05-0.2 \mu \mathrm{g}$ GFP, serving as a transfection marker along with the respective plasmids of interest. At DIV7, neurons were subjected to immunocytochemical staining using the GFP and the cleaved caspase-3 antibodies along with the DNA dye DAPI. GFP-positive neurons were examined for their viability status based on integrity of neurites, nuclear morphology and the apoptotic marker cleaved caspase-3. Analyses were carried out in a blinded manner and approximately 100 cells were analyzed per condition. 


\subsubsection{FBX07 knockout mice}

\subsubsection{Generation of FBXO7 knockout mice}

The generation of FBXO7 conventional knockout mice was achieved using ES cells purchased from the International Knockout Mouse Consortium (EUCOMM ID: 23037). ES cells were injected into C57BL/6N blastocysts, implanted into a female foster mouse and the resulting chimeras crossed to $\mathrm{C} 57 \mathrm{BL} / 6 \mathrm{~N}$ mice to achieve germline transmission.

\subsubsection{Isolation of genomic DNA}

To isolate DNA for genotyping, mouse tail biopsies were digested for $3 \mathrm{~h}$ or overnight at $55^{\circ} \mathrm{C}$ in $200 \mu \mathrm{l}$ of Tail lysis buffer freshly supplemented with $3 \mu$ l proteinase $\mathrm{K}(200 \mu \mathrm{g} / \mathrm{ml})$. After resuspension of the digested tails by agitation of the tube, samples were centrifuged for $7 \mathrm{~min}$ at $14000 \mathrm{rpm}$. The supernatant was then transferred into a new tube and the DNA precipitated using 2.5 volumes of $100 \% \mathrm{EtOH}$. DNA pellets were washed twice with $70 \%$ EtOH and subsequently air-dried prior to addition of $100 \mu \mathrm{l}$ of $\mathrm{H}_{2} \mathrm{O}$.

\subsubsection{Genotyping of FBXO7 knockout mice}

Two separate PCR reactions (Tables 4.9 and 4.10) were set up to achieve genotyping of FBXO7 mice determining presence or absence of the FBXO7 gene or the lacZ reporter cassette, respectively.

Table 4.9: Genotyping PCR mix.

\begin{tabular}{|c|c|}
\hline Amount & Reagent \\
\hline $100 \mathrm{ng}(0.5 \mu \mathrm{l})$ & DNA \\
\hline $1 \mu \mathrm{l}$ & forward primer $(10 \mathrm{pmol} / \mu \mathrm{l})$ \\
\hline $1 \mu \mathrm{l}$ & reverse primer $(10 \mathrm{pmol} / \mu \mathrm{l})$ \\
\hline $2.1 \mu l$ & dNTP $(2.5 \mathrm{mM})$ \\
\hline $0.1 \mu \mathrm{l}$ & GoTaq® DNA Polymerase \\
\hline $5 \mu \mathrm{l}$ & $5 x$ GoTaq ${ }^{\circledR}$ reaction buffer \\
\hline $10.3 \mu \mathrm{l}$ & $\mathrm{H}_{2} \mathrm{O}$ \\
\hline
\end{tabular}


Table 4.10: Genotyping PCR program.

\begin{tabular}{c|rcc|}
\hline Program step & Temperature & Time & Repetitions \\
\hline Initial denaturation & $95^{\circ} \mathrm{C}$ & $3 \mathrm{~min}$ & - \\
Denaturation & $95^{\circ} \mathrm{C}$ & $30 \mathrm{sec}$ & \\
Annealing & $51^{\circ} \mathrm{C}$ & $30 \mathrm{sec}$ & \\
Elongation & $72^{\circ} \mathrm{C}$ & $28 \mathrm{~min}$ & \\
Final elongation & $72^{\circ} \mathrm{C}$ & $10 \mathrm{~min}$ & -
\end{tabular}

\subsubsection{General biochemical methods}

\subsubsection{Tissue lysate preparation}

Mice were sacrificed by $\mathrm{CO}_{2}$ inhalation or cervical dislocation. For general analysis, the isolated tissue of interest was lysed in $\operatorname{Triton}^{T M} X-100$ lysis buffer freshly supplemented with protease, phosphatase and in case of ubiquitin-dependent processes also deubiquitination enzyme inhibitors $(3 \mu \mathrm{g} / \mathrm{ml}$ aprotinin, $1 \mu \mathrm{g} / \mu \mathrm{l}$ leupeptin, $1 \mu \mathrm{g} / \mu \mathrm{l}$ pepstatin, 1 mM DTT; $0.5 \mathrm{mM} \mathrm{NaVO}_{3}, 1 \mathrm{mM} \mathrm{NaF} ; 10 \mathrm{mM} \mathrm{N}$-ethylmaleimide (NEM)). Tissue lysis and homogenization was carried out with a $2 \mathrm{ml}$ dounce (KONTES $®$ ) followed by incubation on ice for $30 \mathrm{~min}$. Subsequently, lysates were centrifuged for $10 \mathrm{~min}$ at $4^{\circ} \mathrm{C}$ and $14000 \mathrm{rpm}$, the supernatant collected in a fresh tube and either directly used for analysis or snap frozen using liquid nitrogen and stored at $-80^{\circ} \mathrm{C}$.

\subsubsection{Cell lysate preparation}

HEK293T or primary neuronal cells were first washed once with PBS, followed by addition of Triton $^{T M}$ X-100 lysis buffer or co-IP buffer freshly supplemented with protease, phosphatase and in case of ubiquitin-dependent processes also deubiquitination enzyme inhibitors. Subsequently, adherent cells were scraped off the plate surface using a cell scraper (Greiner Bio-One) and incubated on ice for $30 \mathrm{~min}$. Subsequently, lysates were centrifuged for $10 \mathrm{~min}$ at $4^{\circ} \mathrm{C}$ and $14000 \mathrm{rpm}$, the supernatant collected in a fresh tube and either directly used for analysis or snap frozen using liquid nitrogen and stored at $-80^{\circ} \mathrm{C}$. 


\subsubsection{Protein concentration determination}

Protein concentration determination of cell or tissue lysates was done using the BioRad Protein Assay, which is based on the method of Bradford (Bradford, 1976). The reagent was diluted 1:5 in $\mathrm{ddH}_{2} \mathrm{O}$ and pipetted into cuvettes (Greiner Bio-One). Subsequently, 1, 3, 5 and $10 \mu \mathrm{g} \mathrm{BSA}$ were added to the mixture serving as a standard curve as well as $1-2 \mu \mathrm{l}$ of the respective lysate of interest followed by vortexing and incubation for 5 min at RT. The absorbance at wavelength $595 \mathrm{~nm}$ was read using a spectrophotometer and the protein concentration calculated according to the BSA standard curve.

\subsubsection{Co-immunoprecipitation (co-IP)}

1 - $2 \mathrm{mg}$ of cell lysates from transfected HEK293T cells were incubated with the primary antibody on a rotator for $3 \mathrm{~h}$ at $4^{\circ} \mathrm{C}$. If different conditions varied in volume, co-IP buffer was used to equalize them. Subsequently, $10-30 \mu$ of Protein A-sepharose bead slurry (Protein A-Sepharose ${ }^{\mathrm{TM}}$ Fast Flow, GE Healthcare), prewashed and maintained in coIP buffer, was added to the samples and incubated for another $45-60 \mathrm{~min}$ at $4^{\circ} \mathrm{C}$ on the rotator. Next, the samples were centrifuged for $30 \mathrm{sec}$ at $7500 \mathrm{rpm}$, the supernatant discarded and the beads washed thrice with $\operatorname{Triton}^{T M} X-100$ lysis buffer followed by one wash with PBS. Finally, the PBS supernatant was removed except for approximately $20 \mu \mathrm{l}$, supplemented with $30 \mu \mathrm{l}$ of SDS-sample buffer and boiled for $5 \mathrm{~min}$ at $95^{\circ} \mathrm{C} .50-100 \mu \mathrm{g}$ of the original cell lysate served as an input control.

\subsubsection{Subcellular fractionation}

Cortical tissue was lysed in Buffer $A$ supplemented with protease inhibitors using a 2 $\mathrm{ml}$ dounce. Next, lysates were centrifuged for $5 \mathrm{~min}$ at $4^{\circ} \mathrm{C}$ and $2000 \mathrm{rpm}$, the supernatant collected (S1) and spun down again for $5 \mathrm{~min}$ at $4^{\circ} \mathrm{C}$ and $2000 \mathrm{rpm}$. The resulting supernatant served as the cytoplasmic fraction. The pellets were washed 6 times with Buffer $A^{+}$and then resuspended in $100 \mu \mathrm{B}$ Buffer $C$ followed by centrifugation for $20 \mathrm{~min}$ at at $4^{\circ} \mathrm{C}$ and $14000 \mathrm{rpm}$. The resulting supernatant was harvested as the nuclear fraction.

\subsubsection{Cell-based ubiquitination assay}

The cell-based ubiquitination assay was carried out using an adapted version of the method described in (Lu et al., 2007). Transfected HEK293T cells were lysed in RIPA buffer 
without SDS supplemented with protease inhibitors and $10 \mathrm{mM} \mathrm{NEM}$. Next, 1 - $2 \mathrm{mg}$ of cell lysate were incubated with $1 \% \operatorname{SDS}$ for $5 \mathrm{~min}$ at $4^{\circ} \mathrm{C}$ on a rotator followed by boiling for 10 $\min$ at $95^{\circ} \mathrm{C}$ to disrupt protein-protein interactions. The SDS concentration was then reduced to $0.1 \%$ by mixing with UA-Lysis Buffer. Subsequently, primary antibody added and incubated overnight at $4^{\circ} \mathrm{C}$ on a rotator. For immunoprecipitation, Protein A-sepharose bead slurry was added and incubated with the samples for $45 \mathrm{~min}$ at $4^{\circ} \mathrm{C}$ on a rotator. Finally, beads were washed twice with UA-Lysis Buffer, once with RIPA containing SDS and once with PBS, followed by addition of $30 \mu \mathrm{l}$ of SDS-sample buffer and boiling for $5 \mathrm{~min}$ at $95^{\circ} \mathrm{C}$. 50 - $100 \mu \mathrm{g}$ of the original cell lysate served as an input control.

\subsubsection{SDS-PAGE and western blot}

SDS-PAGE gels were cast using the Mini-PROTEAN® Tetra Electrophoresis System from Bio-Rad. A standard gel consisted of an upper, so-called stacking gel containing $3.9 \%$ acrylamide, and a lower, so-called separating gel containing 7.5 - $12 \%$ acrylamide depending on the size of the proteins of interest. The composition of the stacking and separating gels is indicated in Table 4.11.

Table 4.11: of gels used for SDS-PAGE.

\begin{tabular}{|c|c|c|c|c|c|}
\hline \multicolumn{2}{|l|}{ Reagent } & \multicolumn{3}{|c|}{ Separating gel } & \multirow{2}{*}{$\begin{array}{c}\text { Stacking gel } \\
3.9 \%\end{array}$} \\
\hline Acrylamide & $7.5 \%$ & $8 \%$ & $10 \%$ & $12 \%$ & \\
\hline ml Acrylamide (30 \% stock) & 1.875 & 2 & 2.5 & 3 & 0.65 \\
\hline ml Lower Buffer & 1.875 & 1.875 & 1.875 & 1.875 & $1.25 \mathrm{ml}$ Upper Buffer \\
\hline $\mathrm{ml} \mathrm{H}{ }_{2} \mathrm{O}$ & 3.75 & 3.625 & 3.125 & 2.625 & 3.05 \\
\hline$\mu \mathrm{l} 10 \%$ APS & \multicolumn{4}{|c|}{75} & 49.5 \\
\hline$\mu \mathrm{l}$ TEMED & \multicolumn{4}{|c|}{7.5} & 4.95 \\
\hline
\end{tabular}

The separating gel reagents were mixed, poured into the gel casting setup and overlaid with a thin layer of isopropanol. After gel polymerization for 10 - $20 \mathrm{~min}$, isopropanol was removed with $\mathrm{H}_{2} \mathrm{O}$ followed by addition of the stacking gel solution, insertion of a 10-well comb and polymerization for another $15 \mathrm{~min}$. 
Boiled SDS sample buffer-added protein samples were loaded into the Running buffer-immersed gel wells along with a protein ladder marker and resolved at 25 - $35 \mathrm{~mA}$ per gel. Following electrophoresis, the SDS-gel was placed onto a nitrocellulose membrane (Amersham ${ }^{\mathrm{TM}}$ Protran $^{\mathrm{TM}}$ ) and the proteins transferred using the Mini Trans-Blot ${ }^{\circledR}$ cell from Bio-Rad in cooled Transfer Buffer for $110 \mathrm{~min}$ at $230 \mathrm{~mA}$. Nitrocellulose membranes were then blocked for 30 min using $4 \%$ milk in PBST followed by three 10 min PBST washes. Subsequently, the membrane was incubated for $1 \mathrm{~h}$ at RT or overnight at $4^{\circ} \mathrm{C}$ with the primary antibody diluted in $3 \%$ BSA or $4 \%$ milk in PBS supplemented with $0.02 \% \mathrm{NaN}_{3}$. Following primary antibody incubation the membranes were washed three times for 10 min with PBST and then subjected to incubation for 30 min at RT with an HRP-coupled secondary antibody diluted in $4 \%$ milk blocking solution. Subsequently, membranes were washed another three times for $10 \mathrm{~min}$ with PBST prior to incubation with an enhanced chemiluminescent $(E C L)$ solution from a peroxidase substrate kit (Pierce ${ }^{T M}$, Thermo Fisher Scientific). Finally, photographic films (Amersham Hyperfilm ${ }^{\mathrm{TM}} \mathrm{ECL}$ ) and an automatic film developer machine (Curix 60, Agfa) were used to visualize the protein bands.

\subsubsection{Colloidal Coomassie staining}

Colloidal Coomassie staining was carried out according to Dörte Hesse's adapted protocol from (Neuhoff et al., 1988). SDS-PAGE gels were fixed with $40 \% \mathrm{v} / \mathrm{v} \mathrm{EtOH,} 10 \%$ $\mathrm{v} / \mathrm{v}$ acetic acid for at least $60 \mathrm{~min}$ at RT. After two washes with $\mathrm{H}_{2} \mathrm{O}$ for $10 \mathrm{~min}$, the gels were incubated in Dye working solution for 60 min or overnight at RT. Subsequently, the gels were washed with $1 \%$ acetic acid until all Coomassie particles were removed.

\subsubsection{Proteasome biochemistry}

\subsubsection{Measurement of proteasome activity from lysates}

The lysate proteasome activity assay was carried out using an adapted version of the method described in (Kisselev and Goldberg, 2005). Transfected HEK293T cells or tissue were lysed and homogenized in the non-detergent-based Proteasome cell lysis buffer $A$ to prevent dissociation of the proteasome holocomplexes and then centrifuged for $10 \mathrm{~min}$ at $4^{\circ} \mathrm{C}$ and $14000 \mathrm{rpm}$. Subsequently, $12 \mu \mathrm{g}$ of tissue or cell lysate was pipetted in triplicates into a 96-well plate containing Proteasome activity assay buffer and incubated for $5 \mathrm{~min}$ at $37^{\circ} \mathrm{C}$. Next, $100 \mu \mathrm{M}$ of the fluorogenic peptide substrate Suc-LLVY-AMC was added to the wells, 
mixed and fluorescence measured at 0, 30, 60, 90 min using a fluorescent plate reader (355 $\mathrm{nm}$ excitation and $460 \mathrm{~nm}$ emission). To verify comparable lysate loading amounts, genotype or successful transfection, $50 \mu \mathrm{g}$ of the respective lysate was subjected to western blot.

\subsubsection{Characterization of proteasome profiles using native PAGE}

Native PAGE gels were cast using the Mini-PROTEAN® Tetra Electrophoresis System from Bio-Rad. The gels consisted of a stacking gel containing $2.75 \%$ acrylamide, and a separating gel containing $3.75 \%$. The composition of the stacking and separating gels is indicated in Table 4.12.

Table 4.12: Composition of gels used for Native PAGE.

\begin{tabular}{c|cc|} 
Reagent & Separating gel & Stacking gel \\
\hline $400 \mathrm{mM}$ Tris base/Boric acid & $1.5 \mathrm{ml}$ & $0.6 \mathrm{ml}$ \\
$1 \mathrm{M} \mathrm{MgCl}_{2}$ & $37.5 \mu \mathrm{l}$ & $15 \mu \mathrm{l}$ \\
$500 \mathrm{mM}$ EDTA & $7.5 \mu \mathrm{l}$ & $3 \mu \mathrm{l}$ \\
$400 \mathrm{mM}$ ATP & $18.75 \mu \mathrm{l}$ & $7.5 \mu \mathrm{l}$ \\
Acrylamide (30\%) & $937.5 \mu \mathrm{l}$ & $275 \mu \mathrm{l}$ \\
$\mathrm{H}_{2} \mathrm{O}$ & $4.98 \mathrm{ml}$ & $2.1 \mathrm{ml}$ \\
APS (10\%) & $75 \mu \mathrm{l}$ & $30 \mu \mathrm{l}$ \\
TEMED & $7.5 \mu \mathrm{l}$ & $3 \mu \mathrm{l}$ \\
\hline
\end{tabular}

The separating gel reagents were mixed, poured into the gel casting setup and overlaid with a thin layer of isopropanol. After gel polymerization for 10 - $20 \mathrm{~min}$, isopropanol was removed with $\mathrm{H}_{2} \mathrm{O}$ followed by addition of the stacking gel solution, insertion of a 10-well comb and polymerization for another $15 \mathrm{~min}$. Gels were then placed into a running chamber placed on ice and filled with cold Native PAGE running buffer.

25 - $75 \mu \mathrm{g}$ of cell or tissue lysates prepared in Proteasome cell lysis buffer A or $25 \mu \mathrm{g}$ of purified 26S proteasomes were mixed with Native PAGE sample buffer and loaded into the gel well. Next, the gels were resolved for $125 \mathrm{~min}$ at $175 \mathrm{~V}$ and afterwards immersed in Native PAGE activity assay buffer. Following addition of $100 \mu \mathrm{M}$ of Suc-LLVY-AMC, the gel was incubated for $10 \mathrm{~min}$ at $37^{\circ} \mathrm{C}$ and the proteasome profile visualized by exposure to UV 
light. For stimulation of the latent $20 \mathrm{~S}$ proteasome activity, $0.02 \%$ SDS was added and the gel incubated for another $10 \mathrm{~min}$ at $37^{\circ} \mathrm{C}$. Subsequently, the gel was immersed in Native PAGE pre-transfer buffer and incubated for $10 \mathrm{~min}$ at RT. Finally, the gel was placed onto a nitrocellulose membrane (Amersham ${ }^{\mathrm{TM}} \operatorname{Protran}^{\mathrm{TM}}$ ) and the proteins transferred using the Mini Trans-Blot $®$ cell from Bio-Rad in cooled Transfer Buffer for $110 \mathrm{~min}$ at $230 \mathrm{~mA}$. Western blot was then carried out to confirm the proteasome content in the gels.

\subsubsection{Glycerol density gradient centrifugation and analysis of proteasome fractions}

Glycerol density gradient centrifugation was carried out using an adapted version of the method described in (Koulich et al., 2008). Transfected HEK293T cells or tissue were lysed and homogenized in Proteasome cell lysis buffer $B$ and then centrifuged for 10 min at $4^{\circ} \mathrm{C}$ and $14000 \mathrm{rpm}$. Per condition, a total amount of $0.5-1.5 \mathrm{mg}$ of lysate was used. Linear glycerol gradients containing 10,20,30 and $40 \%$ glycerol were prepared in a total volume of $4 \mathrm{ml}$ using Glycerol gradient buffer followed by loading of the sample on top. Subsequently, samples were centrifuged for $14 \mathrm{~h}$ at $4^{\circ} \mathrm{C}$ and $83000 \mathrm{~g}$ using a Beckman XL-90 ultracentrifuge with a SW-60 Ti rotor (Beckman Coulter). Following centrifugation, the gradients were pipetted off into 20 fractions with a volume of $200-220 \mu$ l. Per fraction, $20 \mu l$ were subjected to proteasome activity measurement as described in section 4.2.5.1. The remaining fraction was precipitated using a $4 \mathrm{x}$ volume of icecold acetone. Following an incubation time of $60 \mathrm{~min}$ at $-20^{\circ} \mathrm{C}$, the samples were centriuged for $10 \mathrm{~min}$ at $14000 \mathrm{rpm}$ and the supernatant discarded. The pellets were air-dried for $30 \mathrm{~min}$ prior to addition of SDSsample buffer and boiling for $5 \mathrm{~min}$ at $95^{\circ} \mathrm{C}$. SDS-PAGE and western blot were then carried out to confirm protein and proteasome content in each fraction.

\subsubsection{Proteasome affinity purification}

Proteasome affinity purification was carried out using an adapted version of the method described in (Besche and Goldberg, 2012). In this protocol, the GST-tagged Ubldomain of RAD23B is used to bind and precipitate $26 \mathrm{~S}$ proteasomes with high affinity. In a second step, the His ${ }_{10}$-tagged UIM-domain of S5a is used for elution of 26S proteasomes by binding competition for the RAD23B Ubl-domain.

GST-Ubl ${ }^{\text {RAD23B }}$ was previously generated in the lab. To bacterially express and purify $\mathrm{His}_{10}-\mathrm{UIM}^{\mathrm{S5a}}$, the UIM ${ }^{55 a}$ cDNA was cloned into the bacterial expression vector pET28a. For transformation of BL21 bacteria, $50 \mathrm{ng}$ of plasmid was used followed by plating on 
kanamycin-containing $2 x Y T$ agar plates and overnight growth at $37^{\circ} \mathrm{C}$. Next, a preculture of $12 \mathrm{ml} 2 x y T$ with kanamycin was inoculated with a bacterial colony and grown overnight at $37^{\circ} \mathrm{C}$ and $175 \mathrm{rpm} .10 \mathrm{ml}$ of the preculture was then used to adjust the main bacterial culture of $300 \mathrm{ml} 2 \mathrm{x} Y \mathrm{~T}$ and kanamycin to an $\mathrm{OD}_{600}=0.1$. The main culture was then grown at $37^{\circ} \mathrm{C}$ and $175 \mathrm{rpm}$ up to an $\mathrm{OD}_{600}=0.6$ and induced using $0.5 \mathrm{mM}$ Isopropyl $\beta-\mathrm{D}-1$ thiogalactopyranoside (IPTG). At an $\mathrm{OD}_{600}=1.0$ the bacterial culture was spun down for 30 min at $4^{\circ} \mathrm{C}$ and $4000 \mathrm{rpm}$ and resuspended in Resuspension / Lysis buffer freshly supplemented with protease inhibitors $(3 \mu \mathrm{g} / \mathrm{ml}$ aprotinin, $1 \mu \mathrm{g} / \mu \mathrm{l}$ leupeptin, $1 \mu \mathrm{g} / \mu \mathrm{l}$ pepstatin, $150 \mu \mathrm{M}$ phenylmethanesulfonylfluoride (PMSF)). Subsequently, the samples were sonicated three times at $30 \%$ power for $30 \mathrm{sec}$ (SONOPULS UW 2070, Bandelin) followed by centrifugation for $20 \mathrm{~min}$ at $4^{\circ} \mathrm{C}$ and $10000 \mathrm{~g}$. The supernatant was then added with $800 \mu \mathrm{Ni}-$ NTA sepharose bead slurry (Ni-NTA Sepharose ${ }^{\circledR}$, iba lifesciences) and incubated overnight at $4^{\circ} \mathrm{C}$ on a rotator. The beads were then spun down for $1 \mathrm{~min}$ at $4^{\circ} \mathrm{C}$ and $1000 \mathrm{~g}$ followed by one wash of 20x bead volume Resuspension buffer and another wash of 10x bead volume Resuspension buffer with $40 \mathrm{mM}$ imidazole. Elution was achieved by incubation of the beads with Resuspension buffer with $500 \mathrm{mM}$ imidazole overnight at $4^{\circ} \mathrm{C}$ on a rotator. Finally, the eluted fraction was dialyzed twice overnight at $4^{\circ} \mathrm{C}$ in Dialysis buffer and the purification verified by SDS-PAGE and Coomassie blue staining.

For $26 \mathrm{~S}$ proteasome affinity purification, $750 \mathrm{mg}$ of cortical tissue pooled from four P18 FBXO7+/+ and -/- mice, respectively, was lysed in Proteasome lysis buffer C / Purification buffer using a mechanical homogenizer (POLYTRON®, PT 1200 E). Following the centrifugation of the cortical lysates for $20 \mathrm{~min}$ at $4^{\circ} \mathrm{C}$ and $14000 \mathrm{rpm}, 750 \mu \mathrm{g}$ of GST$\mathrm{Ubl}^{\mathrm{RAD23B}}$ and $230 \mu \mathrm{l}$ GST-bead slurry (Glutathione Sepharose ${ }^{\mathrm{TM}} 4 \mathrm{~B}$, GE Healthcare) were added to the lysates and incubated for $2.5 \mathrm{~h}$ at $4^{\circ} \mathrm{C}$ on a rotator. Subsequently, the beads were washed twice with $40 \mathrm{x}$ bead volume Purification buffer prior to the first elution by addition of $750 \mu \mathrm{g}$ of $\mathrm{His}_{10}-\mathrm{UIM}^{\mathrm{S5a}}$. After mixing of the resin by pipetting and incubation for 15 min at $4^{\circ} \mathrm{C}$, the beads were spun down. A second elution was then performed by addition of $750 \mu \mathrm{g}$ of $\mathrm{His}_{10}-\mathrm{UIM}^{\mathrm{S} 5 \mathrm{a}}$. The eluates 1 and 2 were then combined and incubated with $112.5 \mu \mathrm{l}$ Ni-NTA sepharose bead slurry for $30 \mathrm{~min}$ at $4^{\circ} \mathrm{C}$ on a rotator. Following centrifugation for 1.5 min at $4^{\circ} \mathrm{C}$ and $500 \mathrm{rpm}$, the supernatant was collected as the $26 \mathrm{~S}$ proteasome elution fraction. Purity of the $26 \mathrm{~S}$ proteasome eluates was determined by SDS-PAGE and Coomassie blue staining. Purified proteasomes were subjected to further analysis by native PAGE and proteasome activity assays. 


\subsubsection{Histological analysis of mouse brain tissue}

\subsubsection{Transcardial perfusion and fixation}

Mice were anesthetized by intraperitoneal injection of Ketamine/xylazine. Successful anesthesia was assessed by testing twitching reflexes due to pinching of the hind limbs. Subsequently, animals were stabilized on their dorsal side and the abdomen opened using dissection scissors and forceps. Upon removal of the diaphragm, a small butterfly canula (Venoflix ${ }^{\circledR}$, Braun) attached to a peristaltic pump (Heidolph ${ }^{\mathrm{TM}}$ PD 5001, Thermo Fisher Scientific) was inserted into the left ventricle with simultaneous incision making on the right atrium. The blood was then flushed out using PBS, followed by fixation with $4 \%$ PFA (diluted from $8 \%$ PFA with PBS) for tissue destined for vibratome sectioning or $4 \%$ PFA for paraffin $I H C$ for tissue destined for microtome sectioning. The tissues of interest were then harvested and further processed by postfixation. For vibratome sectioning, tissues were postfixed in $4 \%$ PFA for $4 \mathrm{~h}$ at $4^{\circ} \mathrm{C}$, washed once in PBS followed by incubation in a $30 \%$ sucrose solution overnight at $4^{\circ} \mathrm{C}$. For Microtome sectioning, tissues were postfixed in $4 \%$ PFA for paraffin $I H C$ overnight at $4^{\circ} \mathrm{C}$.

\subsubsection{X-Gal staining of vibratome mouse brain sections}

$40 \mu \mathrm{m}$ thick sagittal whole brain sections of P18 FBXO7 +/+ and FBXO7 -/- mice were obtained by vibratome sectioning (VT100S, Leica), collected in PBS and stored in PBS wit $0.02 \% \mathrm{NaN}_{3}$ at $4{ }^{\circ} \mathrm{C}$ until further processing. Free floating sections were then incubated under the exclusion of light overnight at $37^{\circ} \mathrm{C}$ in $X$-gal buffer with freshly added $X-G a l$. Subsequently, sections were washed in PBS and mounted onto microscope slides (Marienfeld), air-dried and mounted in $50 \%$ glycerol. Finally, sections were imaged using a brightfield microscope (Axio Observer Z1, Zeiss) and the Zen 2011 imaging software (Zeiss, Germany).

\subsubsection{Microtome tissue embedding and sectioning}

Tissue destined for microtome sectioning was embedded in paraffin (Paraplast $\circledast$, Leica) using an automated tissue processor (HMP110, MICROM) program listed in Table

\subsection{3.}


Table 4.13: of the automated tissue processor.

\begin{tabular}{c|c|}
\hline Program step & Time \\
\hline $50 \% \mathrm{v} / \mathrm{v} \mathrm{EtOH}$ & $1 \mathrm{~h}$ \\
$70 \% \mathrm{v} / \mathrm{v} \mathrm{EtOH}$ & $2 \times 2 \mathrm{~h}$ \\
$96 \% \mathrm{v} / \mathrm{v} \mathrm{EtOH}$ & $2 \times 2 \mathrm{~h}$ \\
$100 \% \mathrm{v} / \mathrm{v} \mathrm{EtOH}$ & $2 \times 2 \mathrm{~h}$ \\
Isopropanol & $1 \mathrm{~h}$ \\
Xylol & $2 \times 2 \mathrm{~h}$ \\
Paraffin & $2 \times 2 \mathrm{~h}$
\end{tabular}

Subsequently, brains were positioned in metal molds, embedded in $60^{\circ} \mathrm{C}$ warm paraffin and solidified by cooling. Paraffin blocks were then stored at RT until microtome sectioning (HM 430, Thermo Fisher Scientific) of $5 \mu \mathrm{m}$ thick whole brain sagittal or coronal sections of P18 FBXO7+/+ and FBXO7-/- mice. Sections obtained with the sliding microtome were collected and transferred into a waterbath heated to $40^{\circ} \mathrm{C}$ (HIR-3, Kunz Instruments), mounted on microscopy slides (Marienfeld) and then air-dried at RT.

\subsubsection{Immunohistochemical staining of paraffin-embedded sections}

Mounted $5 \mu \mathrm{m}$ thick sections were first subjected to a series of deparaffinization and rehydration steps. For this, sections were incubated for $10 \mathrm{~min}$ at $60^{\circ} \mathrm{C}$ to soften up the paraffin. Paraffin removal was then achieved by two 10 min incubations in Xylol and one 10 min incubation in Xylol//sopropanol (1:2). Subsequently, a series of rehydration steps was carried out by $5 \mathrm{~min}$ incubation in $100 \%, 90 \%, 70 \%$ and $50 \% \mathrm{v} / \mathrm{v} \mathrm{EtOH}$, respectively, concluded by a 5 min incubation in $\mathrm{ddH}_{2} \mathrm{O}$. Subsequently, sections were incubated for $5 \mathrm{~min}$ in Citrate buffer followed by boiling for $10 \mathrm{~min}$ at 650 watts in a microwave for antigen retrieval. Afterwards, sections were cooled down at RT for 30 min and rinsed in Tris buffer with $2 \% \mathrm{w} / \mathrm{v}$ milk powder prior to placement into Shandon ${ }^{\mathrm{TM}}$ coverplates (Thermo Fisher Scientific). Following another rinse in Tris buffer with $2 \% \mathrm{w} / \mathrm{v}$ milk powder, endogenous peroxidase activity was inhibited by treatment with $3 \% \mathrm{H}_{2} \mathrm{O}_{2}$ for 5 min and washing in Tris buffer with $2 \% \mathrm{w} / \mathrm{v}$ milk powder. Sections were blocked by incubation with Blocking buffer for 
10 min at RT and subsequently incubated overnight at $4^{\circ} \mathrm{C}$ in primary antibody diluted in $B S A / P B S$. The next day, sections were rinsed with Tris buffer with $2 \% \mathrm{w} / \mathrm{v}$ milk powder and subjected to staining using the $\mathrm{LSAB}_{2}$ kit (Dako). For this, the slides were first incubated with a biotinylated secondary antibody solution for $10 \mathrm{~min}$, followed by a rinse with Tris buffer with $2 \% \mathrm{w} / \mathrm{v}$ milk powder. Then, slides were incubated for $10 \mathrm{~min}$ with peroxidase-labelled streptavidin and rinsed with Tris buffer. Coverplates were then dismantled and the slides incubated for 5 - 10 min with the HRP substrate chromogen 3-3'-diaminobenzidine (DAB, Dako) resulting in brown-colored precipitates at the sites of antigen localization. Following DAB labelling, the slides were subjected to nuclear staining by rinsing twice in $\mathrm{dd}_{2} \mathrm{O}$ prior to incubation in $0.1 \%$ haematoxylin w/v (Merck) for 15 - 30 sec. Subsequently, sections were rinsed in $\mathrm{dd}_{2} \mathrm{O}$, developed by dipping in $\mathrm{HCl} / \mathrm{Alcohol}$ and incubated for $5 \mathrm{~min}$ with the blueing agent Scott's solution (Thermo Fisher Scientific). After rinsing the slides once more with $d d \mathrm{H}_{2} \mathrm{O}$ a series of dehydration steps was carried out by 5 min incubations in $50 \%, 70 \%$, $90 \%$ and $100 \% \mathrm{v} / \mathrm{v}$ EtOH followed by $10 \mathrm{~min}$ incubations in Xylol/Isopropanol (1:2), Xylol and Xylol, respectively. Finally, samples were coverslipped using Eukitt $®$ (Friedrichs et al.) mounting medium. Image acquisition was done using a brightfield microscope (Axio Observer Z1, Zeiss) and the Zen 2011 imaging software (Zeiss, Germany).

\subsubsection{Labelling of apoptotic cells using the TUNEL assay}

Mounted $5 \mu \mathrm{m}$ thick sections were subjected to the TUNEL assay following the instructions of the commercially available DeadEnd ${ }^{\mathrm{TM}}$ Colorimetric TUNEL System kit (Promega). The assay is based on labelling of fragmented DNA by detection of biotinylated nucleotides at 3'-OH DNA sites incorporated by the terminal deoxynucleotidyl transferase (TdT) enzyme. Samples were coverslipped using Aqua-Poly/Mount (Polysciences) mounting medium. Image acquisition was done using a brightfield microscope (Axio Observer Z1, Zeiss) and the Zen 2011 imaging software (Zeiss, Germany).

\subsubsection{Area quantification of DAB stainings}

Area quantification of GFAP- or Iba1-positive areas was achieved using a customdesigned imageJ macro provided by Dr. Miso Mitkovski. The macro functions by converting the picture into a black and white image, representing DAB-positive areas as black followed by measuring of the percentage area covered by the DAB signal as compared to the total area of interest selected. The detailed script is given below: (Trapp et al., 1998). 


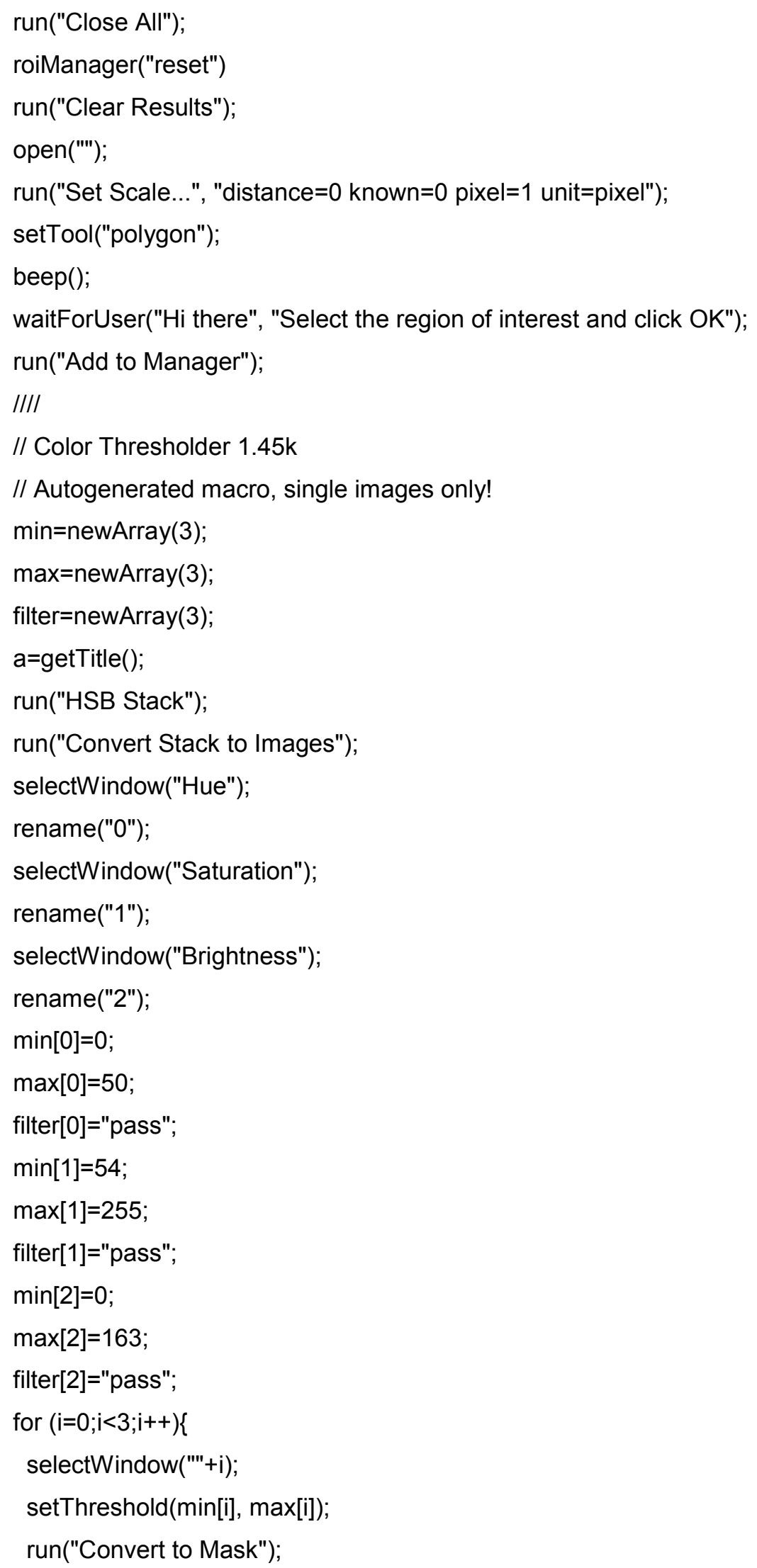




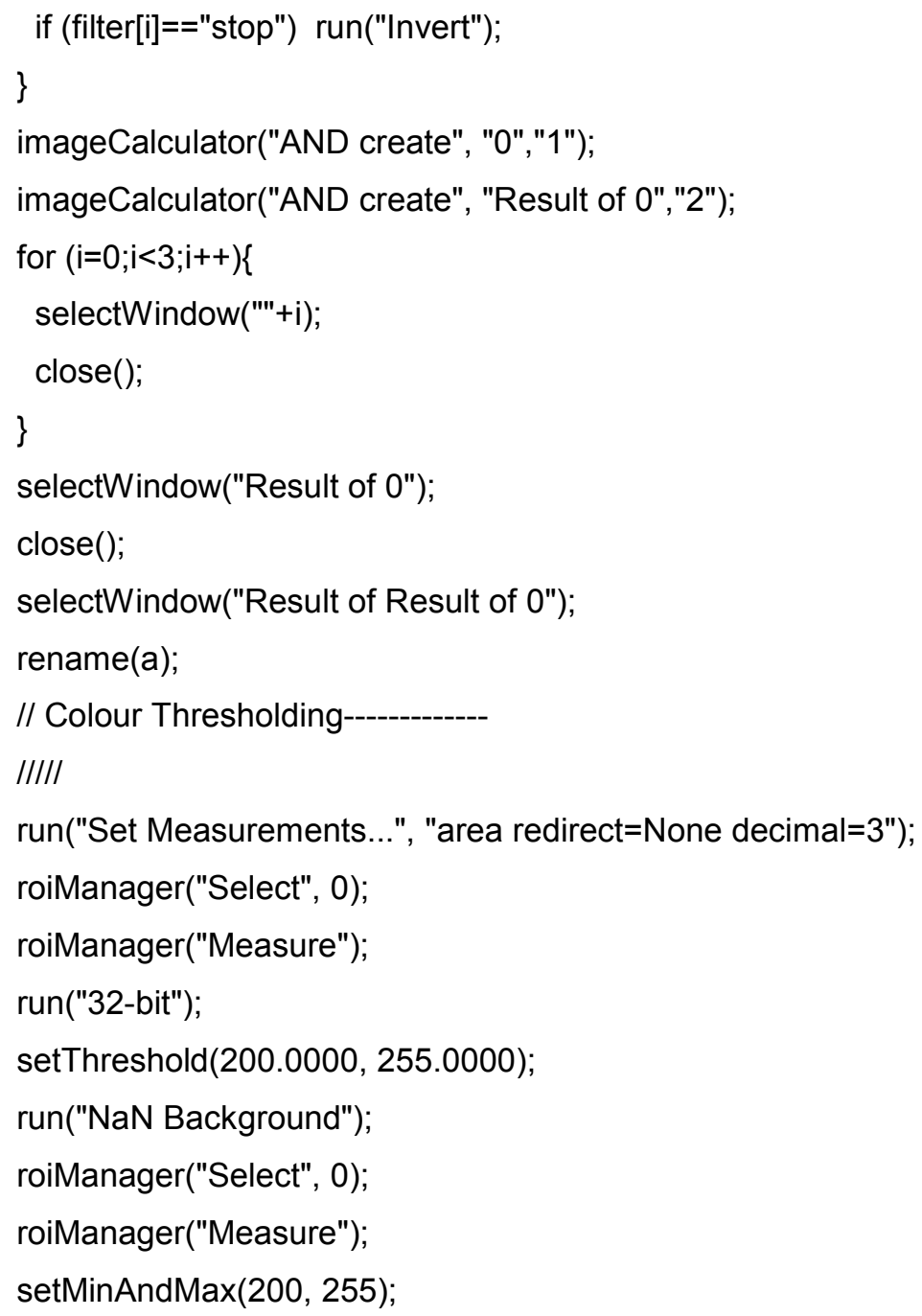

Quantification and analysis was performed in a blinded manner using at least three P18 FBXO7+/+ and FBXO7-/- littermate pairs per staining of interest. 


\section{References}

Abeliovich, A., Schmitz, Y., Farinas, I., Choi-Lundberg, D., Ho, W.H., Castillo, P.E., Shinsky, N., Verdugo, J.M., Armanini, M., Ryan, A., et al. (2000). Mice lacking alpha-synuclein display functional deficits in the nigrostriatal dopamine system. Neuron 25, 239-252.

Amende, I., Kale, A., McCue, S., Glazier, S., Morgan, J.P., and Hampton, T.G. (2005). Gait dynamics in mouse models of Parkinson's disease and Huntington's disease. J Neuroeng Rehabil 2, 20.

Atkin, G., Hunt, J., Minakawa, E., Sharkey, L., Tipper, N., Tennant, W., and Paulson, H.L. (2014). F-box only protein 2 (Fbxo2) regulates amyloid precursor protein levels and processing. J Biol Chem 289, 7038-7048.

Bader, M., Benjamin, S., Wapinski, O.L., Smith, D.M., Goldberg, A.L., and Steller, H. (2011). A conserved $\mathrm{F}$ box regulatory complex controls proteasome activity in Drosophila. Cell 145, 371-382.

Bardien, S., Lesage, S., Brice, A., and Carr, J. (2011). Genetic characteristics of leucine-rich repeat kinase 2 (LRRK2) associated Parkinson's disease. Parkinsonism Relat Disord 17, 501-508.

Beck, F., Unverdorben, P., Bohn, S., Schweitzer, A., Pfeifer, G., Sakata, E., Nickell, S., Plitzko, J.M., Villa, E., Baumeister, W., et al. (2012). Near-atomic resolution structural model of the yeast 26 S proteasome. Proc Natl Acad Sci U S A 109, 14870-14875.

Becker, E.B., and Bonni, A. (2004). Cell cycle regulation of neuronal apoptosis in development and disease. Prog Neurobiol 72, 1-25.

Bedford, L., Hay, D., Devoy, A., Paine, S., Powe, D.G., Seth, R., Gray, T., Topham, I., Fone, K., Rezvani, N., et al. (2008). Depletion of 265 proteasomes in mouse brain neurons causes neurodegeneration and Lewy-like inclusions resembling human pale bodies. J Neurosci 28, 8189-8198.

Besche, H.C., and Goldberg, A.L. (2012). Affinity purification of mammalian 26S proteasomes using an ubiquitin-like domain. Methods Mol Biol 832, 423-432.

Bodner, R.A., Outeiro, T.F., Altmann, S., Maxwell, M.M., Cho, S.H., Hyman, B.T., McLean, P.J., Young, A.B., Housman, D.E., and Kazantsev, A.G. (2006). Pharmacological promotion of inclusion formation: a therapeutic approach for Huntington's and Parkinson's diseases. Proc Natl Acad Sci U S A 103, 4246-4251. 
Bose, S., Stratford, F.L., Broadfoot, K.I., Mason, G.G., and Rivett, A.J. (2004). Phosphorylation of $20 \mathrm{~S}$ proteasome alpha subunit C8 (alpha7) stabilizes the 26S proteasome and plays a role in the regulation of proteasome complexes by gamma-interferon. Biochem $\mathrm{J}$ $378,177-184$.

Bousquet-Dubouch, M.P., Baudelet, E., Guerin, F., Matondo, M., Uttenweiler-Joseph, S., Burlet-Schiltz, O., and Monsarrat, B. (2009). Affinity purification strategy to capture human endogenous proteasome complexes diversity and to identify proteasome-interacting proteins. Mol Cell Proteomics 8, 1150-1164.

Bove, J., Zhou, C., Jackson-Lewis, V., Taylor, J., Chu, Y., Rideout, H.J., Wu, D.C., Kordower, J.H., Petrucelli, L., and Przedborski, S. (2006). Proteasome inhibition and Parkinson's disease modeling. Ann Neurol 60, 260-264.

Bradford, M.M. (1976). A rapid and sensitive method for the quantitation of microgram quantities of protein utilizing the principle of protein-dye binding. Anal Biochem 72, 248-254.

Burchell, V.S., Nelson, D.E., Sanchez-Martinez, A., Delgado-Camprubi, M., Ivatt, R.M., Pogson, J.H., Randle, S.J., Wray, S., Lewis, P.A., Houlden, H., et al. (2013). The Parkinson's disease-linked proteins Fbxo7 and Parkin interact to mediate mitophagy. Nat Neurosci 16, 1257-1265.

Cardozo, T., and Pagano, M. (2004). The SCF ubiquitin ligase: insights into a molecular machine. Nat Rev Mol Cell Biol 5, 739-751.

Chan, N.C., Salazar, A.M., Pham, A.H., Sweredoski, M.J., Kolawa, N.J., Graham, R.L.J., Hess, S., and Chan, D.C. (2011). Broad activation of the ubiquitin-proteasome system by Parkin is critical for mitophagy. Human Molecular Genetics 20, 1726-1737.

Chang, Y.F., Cheng, C.M., Chang, L.K., Jong, Y.J., and Yuo, C.Y. (2006). The F-box protein Fbxo7 interacts with human inhibitor of apoptosis protein clAP1 and promotes clAP1 ubiquitination. Biochem Biophys Res Commun 342, 1022-1026.

Chartier-Harlin, M.C., Kachergus, J., Roumier, C., Mouroux, V., Douay, X., Lincoln, S., Levecque, C., Larvor, L., Andrieux, J., Hulihan, M., et al. (2004). alpha-synuclein locus duplication as a cause of familial Parkinson's disease. Lancet 364, 1167-1169.

Chen, Z., and Pickart, C.M. (1990). A 25-kilodalton ubiquitin carrier protein (E2) catalyzes multi-ubiquitin chain synthesis via lysine 48 of ubiquitin. J Biol Chem 265, 21835-21842.

Cookson, M.R. (2005). The biochemistry of Parkinson's disease. Annu Rev Biochem 74, 2952.

Corti, O., Lesage, S., and Brice, A. (2011). What Genetics Tells Us About the Causes and Mechanisms of Parkinson's Disease. Physiol Rev 91, 1161-1218. 
Coux, O., Tanaka, K., and Goldberg, A.L. (1996). Structure and functions of the $20 \mathrm{~S}$ and $26 \mathrm{~S}$ proteasomes. Annu Rev Biochem 65, 801-847.

Cui, Z., Scruggs, S.B., Gilda, J.E., Ping, P., and Gomes, A.V. (2014). Regulation of cardiac proteasomes by ubiquitination, SUMOylation, and beyond. J Mol Cell Cardiol 71, 32-42.

da Fonseca, P.C., and Morris, E.P. (2008). Structure of the human 26 S proteasome: subunit radial displacements open the gate into the proteolytic core. J Biol Chem 283, 23305-23314.

Damier, P., Hirsch, E.C., Zhang, P., Agid, Y., and Javoy-Agid, F. (1993). Glutathione peroxidase, glial cells and Parkinson's disease. Neuroscience 52, 1-6.

David, Y., Ternette, N., Edelmann, M.J., Ziv, T., Gayer, B., Sertchook, R., Dadon, Y., Kessler, B.M., and Navon, A. (2011). E3 ligases determine ubiquitination site and conjugate type by enforcing specificity on E2 enzymes. J Biol Chem 286, 44104-44115.

Demasi, M., Silva, G.M., and Netto, L.E. (2003). 20 S proteasome from Saccharomyces cerevisiae is responsive to redox modifications and is S-glutathionylated. J Biol Chem 278, 679-685.

Di Fonzo, A., Dekker, M.C., Montagna, P., Baruzzi, A., Yonova, E.H., Correia Guedes, L., Szczerbinska, A., Zhao, T., Dubbel-Hulsman, L.O., Wouters, C.H., et al. (2009). FBXO7 mutations cause autosomal recessive, early-onset parkinsonian-pyramidal syndrome. Neurology 72, 240-245.

Dickson, D.W. (2012). Parkinson's disease and parkinsonism: neuropathology. Cold Spring Harb Perspect Med 2.

Dodson, M.W., and Guo, M. (2007). Pink1, Parkin, DJ-1 and mitochondrial dysfunction in Parkinson's disease. Curr Opin Neurobiol 17, 331-337.

Fabre, B., Lambour, T., Garrigues, L., Amalric, F., Vigneron, N., Menneteau, T., Stella, A., Monsarrat, B., Van den Eynde, B., Burlet-Schiltz, O., et al. (2015). Deciphering preferential interactions within supramolecular protein complexes: the proteasome case. Molecular Systems Biology 11.

Feldman, R.M., Correll, C.C., Kaplan, K.B., and Deshaies, R.J. (1997). A complex of Cdc4p, Skp1p, and Cdc53p/cullin catalyzes ubiquitination of the phosphorylated CDK inhibitor Sic1p. Cell 91, 221-230.

Finley, D. (2009). Recognition and processing of ubiquitin-protein conjugates by the proteasome. Annu Rev Biochem 78, 477-513.

Friedrichs, J., Fink, D., Mauch, C., Kindler, D., Hartmann, W., Schule, R., Buettner, R., and Friedrichs, N. (2014). TGF-beta1-dependent induction and nuclear translocation of FHL2 promotes keratin expression in pilomatricoma. Virchows Arch. 
Fujita, M., Sugama, S., Nakai, M., Takenouchi, T., Wei, J., Urano, T., Inoue, S., and Hashimoto, M. (2007). alpha-Synuclein stimulates differentiation of osteosarcoma cells: relevance to down-regulation of proteasome activity. J Biol Chem 282, 5736-5748.

Funayama, M., Hasegawa, K., Kowa, H., Saito, M., Tsuji, S., and Obata, F. (2002). A new locus for Parkinson's disease (PARK8) maps to chromosome 12p11.2-q13.1. Ann Neurol 51, 296-301.

Furukawa, Y., Vigouroux, S., Wong, H., Guttman, M., Rajput, A.H., Ang, L., Briand, M., Kish, S.J., and Briand, Y. (2002). Brain proteasomal function in sporadic Parkinson's disease and related disorders. Ann Neurol 51, 779-782.

Gautier, C.A., Kitada, T., and Shen, J. (2008). Loss of PINK1 causes mitochondrial functional defects and increased sensitivity to oxidative stress. Proc Natl Acad Sci U S A 105, 1136411369.

Goldberg, M.S., Fleming, S.M., Palacino, J.J., Cepeda, C., Lam, H.A., Bhatnagar, A., Meloni, E.G., Wu, N., Ackerson, L.C., Klapstein, G.J., et al. (2003). Parkin-deficient mice exhibit nigrostriatal deficits but not loss of dopaminergic neurons. J Biol Chem 278, 43628-43635.

Goldberg, M.S., Pisani, A., Haburcak, M., Vortherms, T.A., Kitada, T., Costa, C., Tong, Y., Martella, G., Tscherter, A., Martins, A., et al. (2005). Nigrostriatal dopaminergic deficits and hypokinesia caused by inactivation of the familial Parkinsonism-linked gene DJ-1. Neuron 45, 489-496.

Gong, B., Chen, F., Pan, Y., Arrieta-Cruz, I., Yoshida, Y., Haroutunian, V., and Pasinetti, G.M. (2010). SCFFbx2-E3-ligase-mediated degradation of BACE1 attenuates Alzheimer's disease amyloidosis and improves synaptic function. Aging Cell 9, 1018-1031.

Groll, M., Bajorek, M., Kohler, A., Moroder, L., Rubin, D.M., Huber, R., Glickman, M.H., and Finley, D. (2000). A gated channel into the proteasome core particle. Nat Struct Biol 7, 10621067.

Gunduz, A., Eken, A.G., Bilgic, B., Hanagasi, H.A., Bilguvar, K., Gunel, M., Basak, A.N., and Ertan, S. (2014). FBX07-R498X mutation: Phenotypic variability from chorea to early onset parkinsonism within a family. Parkinsonism Relat D 20,1253-1256.

Hartmann-Petersen, R., and Gordon, C. (2004). Integral UBL domain proteins: a family of proteasome interacting proteins. Semin Cell Dev Biol 15, 247-259.

Hawlitschka, A., Haas, S.J., Schmitt, O., Weiss, D.G., and Wree, A. (2007). Effects of systemic PSI administration on catecholaminergic cells in the brain, adrenal medulla and carotid body in Wistar rats. Brain Res 1173, 137-144.

Hershko, A., and Ciechanover, A. (1998). The ubiquitin system. Annu Rev Biochem 67, 425479. 
Hirsch, E.C. (2007). Animal models in neurodegenerative diseases. J Neural Transm Suppl, 87-90.

Hsu, J.-M., Lee, Y.-C.G., Yu, C.-T.R., and Huang, C.-Y.F. (2004). Fbx7 Functions in the SCF Complex Regulating Cdk1-Cyclin B-phosphorylated Hepatoma Up-regulated Protein (HURP) Proteolysis by a Proline-rich Region. Journal of Biological Chemistry 279, 32592-32602.

Ikeda, F., and Dikic, I. (2008). Atypical ubiquitin chains: new molecular signals. 'Protein Modifications: Beyond the Usual Suspects' review series. EMBO Rep 9, 536-542.

Imai, Y., Soda, M., and Takahashi, R. (2000). Parkin suppresses unfolded protein stressinduced cell death through its E3 ubiquitin-protein ligase activity. Journal of Biological Chemistry 275, 35661-35664.

Jin, J., Cardozo, T., Lovering, R.C., Elledge, S.J., Pagano, M., and Harper, J.W. (2004). Systematic analysis and nomenclature of mammalian F-box proteins. Genes Dev 18, 25732580.

Kato, H., Araki, T., Imai, Y., Takahashi, A., and Itoyama, Y. (2003). Protection of dopaminergic neurons with a novel astrocyte modulating agent (R)-(-)-2-propyloctanoic acid (ONO-2506) in an MPTP-mouse model of Parkinson's disease. J Neurol Sci 208, 9-15.

Kett, L.R., Stiller, B., Bernath, M.M., Tasset, I., Blesa, J., Jackson-Lewis, V., Chan, R.B., Zhou, B., Di Paolo, G., Przedborski, S., et al. (2015). alpha-Synuclein-independent histopathological and motor deficits in mice lacking the endolysosomal Parkinsonism protein Atp13a2. J Neurosci 35, 5724-5742.

Kirk, R., Laman, H., Knowles, P.P., Murray-Rust, J., Lomonosov, M., Meziane el, K., and McDonald, N.Q. (2008). Structure of a conserved dimerization domain within the F-box protein Fbxo7 and the PI31 proteasome inhibitor. J Biol Chem 283, 22325-22335.

Kish-Trier, E., and Hill, C.P. (2013). Structural biology of the proteasome. Annu Rev Biophys 42, 29-49.

Kisselev, A.F., Akopian, T.N., Castillo, V., and Goldberg, A.L. (1999). Proteasome active sites allosterically regulate each other, suggesting a cyclical bite-chew mechanism for protein breakdown. Molecular Cell 4, 395-402.

Kisselev, A.F., and Goldberg, A.L. (2005). Monitoring activity and inhibition of 265 proteasomes with fluorogenic peptide substrates. Methods Enzymol 398, 364-378.

Kitada, T., Asakawa, S., Hattori, N., Matsumine, H., Yamamura, Y., Minoshima, S., Yokochi, M., Mizuno, Y., and Shimizu, N. (1998). Mutations in the parkin gene cause autosomal recessive juvenile parkinsonism. Nature 392, 605-608. 
Konishi, Y., Stegmuller, J., Matsuda, T., Bonni, S., and Bonni, A. (2004). Cdh1-APC controls axonal growth and patterning in the mammalian brain. Science 303, 1026-1030.

Kordower, J.H., Kanaan, N.M., Chu, Y., Suresh Babu, R., Stansell, J., 3rd, Terpstra, B.T., Sortwell, C.E., Steece-Collier, K., and Collier, T.J. (2006). Failure of proteasome inhibitor administration to provide a model of Parkinson's disease in rats and monkeys. Ann Neurol 60, 264-268.

Koulich, E., Li, X., and DeMartino, G.N. (2008). Relative structural and functional roles of multiple deubiquitylating proteins associated with mammalian $26 \mathrm{~S}$ proteasome. Mol Biol Cell 19, 1072-1082.

Kruger, R., Kuhn, W., Muller, T., Woitalla, D., Graeber, M., Kosel, S., Przuntek, H., Epplen, J.T., Schols, L., and Riess, O. (1998). Ala30Pro mutation in the gene encoding alphasynuclein in Parkinson's disease. Nat Genet 18, 106-108.

Kuiken, H.J., Egan, D.A., Laman, H., Bernards, R., Beijersbergen, R.L., and Dirac, A.M. (2012). Identification of F-box only protein 7 as a negative regulator of NF-kappaB signaling. Journal of Cellular and Molecular Medicine, n/a-n/a.

Laman, H. (2006). Fbxo7 gets proactive with cyclin D/cdk6. Cell Cycle 5, 279-282.

Lander, G.C., Estrin, E., Matyskiela, M.E., Bashore, C., Nogales, E., and Martin, A. (2012). Complete subunit architecture of the proteasome regulatory particle. Nature 482, 186-191.

Langston, J.W., Irwin, I., Langston, E.B., and Forno, L.S. (1984). 1-Methyl-4-phenylpyridinium ion (MPP+): identification of a metabolite of MPTP, a toxin selective to the substantia nigra. Neurosci Lett 48, 87-92.

Lin, C.H., Chen, M.L., Lai, T.T., Tai, C.H., and Wu, R.M. (2013). Mutational analysis of FBXO7 gene in Parkinson's disease in a Taiwanese population. Neurobiol Aging 34, 1713 e1711-1714.

Litterman, N., Ikeuchi, Y., Gallardo, G., O'Connell, B.C., Sowa, M.E., Gygi, S.P., Harper, J.W., and Bonni, A. (2011). An OBSL1-Cul7Fbxw8 ubiquitin ligase signaling mechanism regulates Golgi morphology and dendrite patterning. PLoS Biol 9, e1001060.

Liu, L., and McKeehan, W.L. (2002). Sequence analysis of LRPPRC and its SEC1 domain interaction partners suggests roles in cytoskeletal organization, vesicular trafficking, nucleocytosolic shuttling, and chromosome activity. Genomics 79, 124-136.

Liu, L., Vo, A., Liu, G., and McKeehan, W.L. (2005). Distinct structural domains within C19ORF5 support association with stabilized microtubules and mitochondrial aggregation and genome destruction. Cancer Res 65, 4191-4201. 
Livnat-Levanon, N., Kevei, E., Kleifeld, O., Krutauz, D., Segref, A., Rinaldi, T., Erpapazoglou, Z., Cohen, M., Reis, N., Hoppe, T., et al. (2014). Reversible 26S Proteasome Disassembly upon Mitochondrial Stress. Cell Reports 7, 1371-1380.

Lohmann, E., Coquel, A.S., Honore, A., Gurvit, H., Hanagasi, H., Emre, M., Leutenegger, A.L., Drouet, V., Sahbatou, M., Guven, G., et al. (2015). A new F-box protein 7 gene mutation causing typical Parkinson's disease. Mov Disord 30, 1130-1133.

Lomonosov, M., Meziane el, K., Ye, H., Nelson, D.E., Randle, S.J., and Laman, H. (2011). Expression of $\mathrm{Fbxo7}$ in haematopoietic progenitor cells cooperates with p53 loss to promote lymphomagenesis. PLoS ONE 6, e21165.

Lowe, J., Blanchard, A., Morrell, K., Lennox, G., Reynolds, L., Billett, M., Landon, M., and Mayer, R.J. (1988). Ubiquitin Is a Common Factor in Intermediate Filament Inclusion-Bodies of Diverse Type in Man, Including Those of Parkinsons-Disease, Picks Disease, and Alzheimers-Disease, as Well as Rosenthal Fibers in Cerebellar Astrocytomas, Cytoplasmic Bodies in Muscle, and Mallory Bodies in Alcoholic Liver-Disease. Journal of Pathology 155, 915.

Lu, C., Pribanic, S., Debonneville, A., Jiang, C., and Rotin, D. (2007). The PY motif of ENaC, mutated in Liddle syndrome, regulates channel internalization, sorting and mobilization from subapical pool. Traffic 8, 1246-1264.

Luders, J., Demand, J., and Hohfeld, J. (2000). The ubiquitin-related BAG-1 provides a link between the molecular chaperones Hsc70/Hsp70 and the proteasome. J Biol Chem 275, 4613-4617.

Markey, S.P., Johannessen, J.N., Chiueh, C.C., Burns, R.S., and Herkenham, M.A. (1984). Intraneuronal Generation of a Pyridinium Metabolite May Cause Drug-Induced Parkinsonism. Nature 311, 464-467.

McCutchen-Maloney, S.L., Matsuda, K., Shimbara, N., Binns, D.D., Tanaka, K., Slaughter, C.A., and DeMartino, G.N. (2000). cDNA cloning, expression, and functional characterization of PI31, a proline-rich inhibitor of the proteasome. J Biol Chem 275, 18557-18565.

McNaught, K.S., Belizaire, R., Isacson, O., Jenner, P., and Olanow, C.W. (2003). Altered proteasomal function in sporadic Parkinson's disease. Exp Neurol 179, 38-46.

McNaught, K.S., Bjorklund, L.M., Belizaire, R., Isacson, O., Jenner, P., and Olanow, C.W. (2002a). Proteasome inhibition causes nigral degeneration with inclusion bodies in rats. Neuroreport 13, 1437-1441.

McNaught, K.S., Mytilineou, C., Jnobaptiste, R., Yabut, J., Shashidharan, P., Jennert, P., and Olanow, C.W. (2002b). Impairment of the ubiquitin-proteasome system causes dopaminergic cell death and inclusion body formation in ventral mesencephalic cultures. J Neurochem 81, 301-306. 
Mori, H., Kondo, T., Yokochi, M., Matsumine, H., Nakagawa-Hattori, Y., Miyake, T., Suda, K., and Mizuno, Y. (1998). Pathologic and biochemical studies of juvenile parkinsonism linked to chromosome 6q. Neurology 51, 890-892.

Narendra, D., Tanaka, A., Suen, D.F., and Youle, R.J. (2008). Parkin is recruited selectively to impaired mitochondria and promotes their autophagy. Journal of Cell Biology 183, 795803.

Nelson, D.E., Randle, S.J., and Laman, H. (2013). Beyond ubiquitination: the atypical functions of Fbxo7 and other F-box proteins. Open Biol 3, 130131.

Neuhoff, V., Arold, N., Taube, D., and Ehrhardt, W. (1988). Improved staining of proteins in polyacrylamide gels including isoelectric focusing gels with clear background at nanogram sensitivity using Coomassie Brilliant Blue G-250 and R-250. Electrophoresis 9, 255-262.

Nishikawa, K., Li, H., Kawamura, R., Osaka, H., Wang, Y.L., Hara, Y., Hirokawa, T., Manago, Y., Amano, T., Noda, M., et al. (2003). Alterations of structure and hydrolase activity of parkinsonism-associated human ubiquitin carboxyl-terminal hydrolase L1 variants. Biochem Biophys Res Commun 304, 176-183.

Noiges, R., Eichinger, R., Kutschera, W., Fischer, I., Nemeth, Z., Wiche, G., and Propst, F. (2002). Microtubule-associated protein 1A (MAP1A) and MAP1B: light chains determine distinct functional properties. J Neurosci 22, 2106-2114.

Osaka, H., Wang, Y.L., Takada, K., Takizawa, S., Setsuie, R., Li, H., Sato, Y., Nishikawa, K., Sun, Y.J., Sakurai, M., et al. (2003). Ubiquitin carboxy-terminal hydrolase L1 binds to and stabilizes monoubiquitin in neuron. Hum Mol Genet 12, 1945-1958.

Paisan-Ruiz, C., Guevara, R., Federoff, M., Hanagasi, H., Sina, F., Elahi, E., Schneider, S.A., Schwingenschuh, P., Bajaj, N., Emre, M., et al. (2010). Early-onset L-dopa-responsive parkinsonism with pyramidal signs due to ATP13A2, PLA2G6, FBXO7 and spatacsin mutations. Mov Disord 25, 1791-1800.

Pathare, G.R., Nagy, I., Bohn, S., Unverdorben, P., Hubert, A., Korner, R., Nickell, S., Lasker, K., Sali, A., Tamura, T., et al. (2012). The proteasomal subunit Rpn6 is a molecular clamp holding the core and regulatory subcomplexes together. Proc Natl Acad Sci U S A 109, 149154.

Periquet, M., Latouche, M., Lohmann, E., Rawal, N., De Michele, G., Ricard, S., Teive, H., Fraix, V., Vidailhet, M., Nicholl, D., et al. (2003). Parkin mutations are frequent in patients with isolated early-onset parkinsonism. Brain 126, 1271-1278.

Petroski, M.D., and Deshaies, R.J. (2005). Mechanism of lysine 48-linked ubiquitin-chain synthesis by the cullin-RING ubiquitin-ligase complex SCF-Cdc34. Cell 123, 1107-1120. 
Petrucelli, L., O'Farrell, C., Lockhart, P.J., Baptista, M., Kehoe, K., Vink, L., Choi, P., Wolozin, B., Farrer, M., Hardy, J., et al. (2002). Parkin protects against the toxicity associated with mutant alpha-synuclein: proteasome dysfunction selectively affects catecholaminergic neurons. Neuron 36, 1007-1019.

Pickart, C.M., and Eddins, M.J. (2004). Ubiquitin: structures, functions, mechanisms. Biochim Biophys Acta 1695, 55-72.

Polymeropoulos, M.H., Higgins, J.J., Golbe, L.I., Johnson, W.G., Ide, S.E., Di lorio, G., Sanges, G., Stenroos, E.S., Pho, L.T., Schaffer, A.A., et al. (1996). Mapping of a gene for Parkinson's disease to chromosome 4q21-q23. Science 274, 1197-1199.

Polymeropoulos, M.H., Lavedan, C., Leroy, E., Ide, S.E., Dehejia, A., Dutra, A., Pike, B., Root, H., Rubenstein, J., Boyer, R., et al. (1997). Mutation in the alpha-synuclein gene identified in families with Parkinson's disease. Science 276, 2045-2047.

Poulopoulos, M., Levy, O.A., and Alcalay, R.N. (2012). The neuropathology of genetic Parkinson's disease. Mov Disord 27, 831-842.

Randle, S.J., Nelson, D.E., Patel, S.P., and Laman, H. (2015). Defective erythropoiesis in a mouse model of reduced Fbxo7 expression due to decreased p27 expression. J Pathol.

Rideout, H.J., Lang-Rollin, I.C., Savalle, M., and Stefanis, L. (2005). Dopaminergic neurons in rat ventral midbrain cultures undergo selective apoptosis and form inclusions, but do not upregulate iHSP70, following proteasomal inhibition. J Neurochem 93, 1304-1313.

Sakata, E., Yamaguchi, Y., Kurimoto, E., Kikuchi, J., Yokoyama, S., Yamada, S., Kawahara, H., Yokosawa, H., Hattori, N., Mizuno, Y., et al. (2003). Parkin binds the Rpn10 subunit of $26 \mathrm{~S}$ proteasomes through its ubiquitin-like domain. EMBO Rep 4, 301-306.

Scheffner, M., Nuber, U., and Huibregtse, J.M. (1995). Protein ubiquitination involving an E1E2-E3 enzyme ubiquitin thioester cascade. Nature 373, 81-83.

Schoenfeld, T.A., and Obar, R.A. (1994). Diverse distribution and function of fibrous microtubule-associated proteins in the nervous system. Int Rev Cytol 151, 67-137.

Seemuller, E., Lupas, A., Stock, D., Lowe, J., Huber, R., and Baumeister, W. (1995). Proteasome from Thermoplasma acidophilum: a threonine protease. Science 268, 579-582.

Sherer, T.B., Chowdhury, S., Peabody, K., and Brooks, D.W. (2012). Overcoming obstacles in Parkinson's disease. Mov Disord 27, 1606-1611.

Shojaee, S., Sina, F., Banihosseini, S.S., Kazemi, M.H., Kalhor, R., Shahidi, G.A., FakhraiRad, H., Ronaghi, M., and Elahi, E. (2008). Genome-wide linkage analysis of a Parkinsonianpyramidal syndrome pedigree by 500 K SNP arrays. Am J Hum Genet 82, 1375-1384. 
Singleton, A.B., Farrer, M., Johnson, J., Singleton, A., Hague, S., Kachergus, J., Hulihan, M., Peuralinna, T., Dutra, A., Nussbaum, R., et al. (2003). alpha-Synuclein locus triplication causes Parkinson's disease. Science 302, 841.

Skowyra, D., Craig, K.L., Tyers, M., Elledge, S.J., and Harper, J.W. (1997). F-box proteins are receptors that recruit phosphorylated substrates to the SCF ubiquitin-ligase complex. Cell 91, 209-219.

Solano, S.M., Miller, D.W., Augood, S.J., Young, A.B., and Penney, J.B., Jr. (2000). Expression of alpha-synuclein, parkin, and ubiquitin carboxy-terminal hydrolase L1 mRNA in human brain: genes associated with familial Parkinson's disease. Ann Neurol 47, 201-210.

Spillantini, M.G., Schmidt, M.L., Lee, V.M., Trojanowski, J.Q., Jakes, R., and Goedert, M. (1997). Alpha-synuclein in Lewy bodies. Nature 388, 839-840.

Sun, F., Anantharam, V., Latchoumycandane, C., Kanthasamy, A., and Kanthasamy, A.G. (2005). Dieldrin induces ubiquitin-proteasome dysfunction in alpha-synuclein overexpressing dopaminergic neuronal cells and enhances susceptibility to apoptotic cell death. J Pharmacol Exp Ther 315, 69-79.

Tanaka, A., Cleland, M.M., Xu, S., Narendra, D.P., Suen, D.F., Karbowski, M., and Youle, R.J. (2010). Proteasome and p97 mediate mitophagy and degradation of mitofusins induced by Parkin. J Cell Biol 191, 1367-1380.

Tian, G., Park, S., Lee, M.J., Huck, B., McAllister, F., Hill, C.P., Gygi, S.P., and Finley, D. (2011). An asymmetric interface between the regulatory and core particles of the proteasome. Nature Structural \& Molecular Biology 18, 1259-U1108.

Togel, M., Wiche, G., and Propst, F. (1998). Novel features of the light chain of microtubuleassociated protein MAP1B: microtubule stabilization, self interaction, actin filament binding, and regulation by the heavy chain. J Cell Biol 143, 695-707.

Tomko, R.J., Jr., and Hochstrasser, M. (2013). Molecular architecture and assembly of the eukaryotic proteasome. Annu Rev Biochem 82, 415-445.

Trapp, B.D., Peterson, J., Ransohoff, R.M., Rudick, R., Mork, S., and Bo, L. (1998). Axonal transection in the lesions of multiple sclerosis. N Engl J Med 338, 278-285.

Vadhvani, M., Schwedhelm-Domeyer, N., Mukherjee, C., and Stegmuller, J. (2013). The centrosomal E3 ubiquitin ligase FBXO31-SCF regulates neuronal morphogenesis and migration. PLoS ONE 8, e57530.

Verma, R., Chen, S., Feldman, R., Schieltz, D., Yates, J., Dohmen, J., and Deshaies, R.J. (2000). Proteasomal proteomics: identification of nucleotide-sensitive proteasome-interacting proteins by mass spectrometric analysis of affinity-purified proteasomes. Mol Biol Cell 11, 3425-3439. 
Wang, X., Yen, J., Kaiser, P., and Huang, L. (2010). Regulation of the 26 S proteasome complex during oxidative stress. Sci Signal 3, ra88.

Wilkinson, K.D., Deshpande, S., and Larsen, C.N. (1992). Comparisons of neuronal (PGP 9.5) and non-neuronal ubiquitin C-terminal hydrolases. Biochem Soc Trans 20, 631-637.

Wilkinson, K.D., Lee, K., Deshpande, S., Duerksenhughes, P., Boss, J.M., and Pohl, J. (1989). The Neuron-Specific Protein Pgp-9.5 Is a Ubiquitin Carboxyl-Terminal Hydrolase. Science 246, 670-673.

Wyss-Coray, T., and Mucke, L. (2002). Inflammation in neurodegenerative disease--a doubleedged sword. Neuron 35, 419-432.

Yalcin-Cakmakli, G., Olgiati, S., Quadri, M., Breedveld, G.J., Cortelli, P., Bonifati, V., and Elibol, B. (2014). A new Turkish family with homozygous FBXO7 truncating mutation and juvenile atypical parkinsonism. Parkinsonism Relat Disord 20, 1248-1252.

Yang, Y., Nishimura, I., Imai, Y., Takahashi, R., and Lu, B. (2003). Parkin suppresses dopaminergic neuron-selective neurotoxicity induced by Pael-R in Drosophila. Neuron 37, 911-924.

Yonashiro, R., Kimijima, Y., Shimura, T., Kawaguchi, K., Fukuda, T., Inatome, R., and Yanagi, S. (2012). Mitochondrial ubiquitin ligase MITOL blocks S-nitrosylated MAP1B-light chain 1-mediated mitochondrial dysfunction and neuronal cell death. Proc Natl Acad Sci U S A 109, 2382-2387.

Zarranz, J.J., Alegre, J., Gomez-Esteban, J.C., Lezcano, E., Ros, R., Ampuero, I., Vidal, L., Hoenicka, J., Rodriguez, O., Atares, B., et al. (2004). The new mutation, E46K, of alphasynuclein causes Parkinson and Lewy body dementia. Ann Neurol 55, 164-173.

Zhao, T., De Graaff, E., Breedveld, G.J., Loda, A., Severijnen, L.A., Wouters, C.H., Verheijen, F.W., Dekker, M.C., Montagna, P., Willemsen, R., et al. (2011). Loss of nuclear activity of the FBXO7 protein in patients with parkinsonian-pyramidal syndrome (PARK15). PLoS ONE 6, e16983. 


\section{Acknowledgements}

First and foremost, I would like to express my sincere gratitude to my advisor Dr. Judith Stegmüller for her support and trust in my abilities throughout the Ph.D. project. It was a pleasure to develop as a scientist in her lab and provided me with invaluable experiences for the future.

I thank my thesis committee members Prof. Dr. Tiago Fleming Outeiro and Prof. Dr. Klaus-Armin Nave for their valuable advice and critical evaluation of my project during the regular meetings.

Also, I would like to thank Prof. Dr. Michael Hörner and Sandra Drube for their immense support and for the truly encouraging and inspiring atmosphere in the IMPRS Neuroscience program.

Moreover, my scientific and personal development was majorly contributed to by the many and very diverse people I met and interacted with in Göttingen.

I would like to thank the AG Simons group members for being at my side in the race of the daily Klinikum-Mensa-survival. In particular, I would like to thank Nic for the many scientific but also RTS-related discussions. En Taro Adun. My special thanks go to Paula, Ludo and FloridaMcKenzie, two and a half souls that joined me on the countless and quite literally dark hours of this journey. Also, I am very grateful to Nic, Ludo and Paula for lastminute thesis corrections, advice and support.

Furthermore I would like to thank my bro Bekir, whom I could always count on scientifically and personally since the initial time of our Master's. Many thanks also to my friends "from the other side", Adam and Diana, for their support and frequent tasting of international delicacies.

Of course I would like to thank the people making life in the lab exciting and experienceful. Thank you Madhu, Mayur, Annika and Anna for guiding the way as seniors. Many thanks to Shi-Ju for the tons of fun, endurance of the tough irony classes and generation of unconventional proverbs. Thank you Sabitha and Guergana for being patient students and being the new hope of the lab. Special thanks to my baymate Nicola, who has seen me grow up in the lab and supported me ever since. In particular, I would like to thank Siv for being a fantastic project mate and for the many hours of Debattierclub Göttingen. 
Furthermore, I would like to thank Chaitali for her constant support and valuable advice during all of this time.

Finally, I would like to thank my parents for having faith in me and everything they have done for me. 


\section{Appendix 1}

List of plasmid constructs used in this study along with respective identification numbers, if applicable:

Table A: List of plasmids.

\begin{tabular}{|c|c|c|}
\hline Vector plasmid & Constructed by & Primer numbers \\
\hline pEGFP-C1-FBXO7 & Madhuvanthi Kannan & 15222,15223 \\
\hline pSUPER-shFBXO7 \#1 & Siv Vingill & A \\
\hline pSUPER-shFBXO7 \#4 & Siv Vingill & B \\
\hline pCMV-myc-FBXO7 WT & Madhuvanthi Kannan & 15222,15223 \\
\hline pCMV-myc-FBXO7 T22M & Siv Vingill & 23795, 23796 \\
\hline pCMV-myc FBXO7 M115I & Siv Vingill & 23797,23798 \\
\hline pCMV-myc-FBXO7 R378G & Siv Vingill & 23799,23800 \\
\hline pCMV-myc-FBXO7 R498X & Siv Vingill & 23801, 23802 \\
\hline pCMV-myc-FBXO7 NT & Siv Vingill & 15223,28045 \\
\hline pCMV-myc-FBXO7 Ubl & Siv Vingill & $29150-2,25771$ \\
\hline pCMV-myc-FBXO7 FP & Siv Vingill & 28506,28507 \\
\hline pCMV-myc-FBXO7 CT & Siv Vingill & 15222, 25771 \\
\hline pCMV-myc-FBXO7 Fbox & Siv Vingill & 28504,28505 \\
\hline pCMV-myc-FBXO7 PRR & Siv Vingill & 28044,25771 \\
\hline pEGFP-C1-PSMA2 & David Brockelt & 30490,28647 \\
\hline p3xFLAG-CMV-10-PSMA2 & David Brockelt & 29139,28647 \\
\hline pCMV10-3xFLAG-LC1 WT & David Brockelt & 28482,28646 \\
\hline pCMV10-3xFLAG-LC1 MTB & David Brockelt & 28482,28927 \\
\hline pCMV10-3xFLAG-LC1 AB & David Brockelt & 28928,28646 \\
\hline
\end{tabular}


\begin{tabular}{l|l} 
Appendix 1 & viii
\end{tabular}

\begin{tabular}{c|cc|}
\hline $\begin{array}{c}\text { pCMV10-3xFLAG-LC1 } \\
\text { MAGD }\end{array}$ & David Brockelt & 28482, 28930, 28929, 28646 \\
pCMV10-3xFLAG-LC2 & David Brockelt & 29441,29442 \\
pCMV10-3xFLAG-MAP1S & David Brockelt & 29439,29440 \\
pET-28a-UIM2 & David Brockelt & 33589,33590 \\
pGEX-Ubl ${ }^{\text {RAD23B }}$ & David Brockelt & 31340,31341 \\
pGBT9-FBXO7 & David Brockelt & 25769,25771 \\
pCMV-myc-FBXO31 & Dr. Raman Kumar, Prof. David & University of Adelaide, \\
pRK HA Ub WT & Callen & Australia \\
pRK HA Ub K63R & Hiroshi Kawabe & MPI-EM, Göttingen \\
pRK HA Ub K48R & Hiroshi Kawabe & MPI-EM, Göttingen \\
\hline
\end{tabular}




\section{Appendix 2}

List of primer sequences used in this study along with respective identification numbers, if applicable:

Table B: List of primers.

Primer number

15222

15223

A

B

23795

23796

23797

23798

23799

23800

23801

23802

28045

28044

29150-2

25769

25771

29139

28647
Sequence $5^{\prime}-3^{\prime}$

ATATGAATTCATATGAGGCTGCGGGTG

ATATGGTACCTCACATGAATGACAGCCGG

GAAGAGACCTTGGCTTCATA

GAAACTACGCATCTTCCGAC

CCCGAGACGGAGCCGATGCTGGGGCATTTGCGC

GCGCAAATGCCCCAGCATCGGCTCCGTCTCGGG

TCCAATCAGACTAGCATCCAGGATGAACAACCA

TGGTTGTTCATCCTGGATGCTAGTCTGATTGGA

AGGTTTTTATATCTGGGTGATTTTCGAGACAAT

ATTGTCTCGAAAATCACCCAGATATAAAAACCT

CCCATCTTGCCAGGGTGAGGCGGCCCCAATGAC

GTCATTGGGGCCGCCTCACCCTGGCAAGATGGG

ATAGAATTCATGCCAGATGTATTTGGGTTGGTC

CTCGCGGCCGCTCAGTCTCGAAAATCACGCAGATA

ATAGAATTCATATGCCAGCGCCTAATATACCTTCATCC

ATAGAATTCATGAGGCTGCGGGTGCGG

CTCGTCGACTCACATGAATGACAGCCGGCCATC

TATAGCGGCCGCAATGGCGGAGCGCGGGTACA

CTCGGATCCTTATGCTATGGCAGCCAAGT 


\begin{tabular}{|c|c|}
\hline 28482 & ATAAAGCTTATGGACAAAACTGTCACGTACAAACACA \\
\hline 28646 & CTCGGATCCTTACAGTTCAATCTTGCATGCAG \\
\hline 28927 & CTCGGATCCTTAAGTTCCTGGTCCTGCAGTGGC \\
\hline 28928 & ATAAAGCTTATGACTGCAGGACCAGGAACTACC \\
\hline 28930 & AAAGCGTCCAGGACATTCCACATCAACATT \\
\hline 28929 & AATGTTGATGTGGAATGTCCTGGACGCTTT \\
\hline 29441 & ATAAAGCTTATGCCCCGCCCATCСССТCCC \\
\hline 29442 & CTCGTCGACTCAGAACTCAATCTTGCAGGCAGGG \\
\hline 29439 & ATAAAGCTTATGGCGGCGGTGGCTGGATC \\
\hline 29440 & CTCGTCGACCTAGAACTCCACCTTGCAGGCC \\
\hline 30490 & ATAGAATTCGATGGCGGAGCGCGGGTACA \\
\hline 31340 & TATGAATTCATGCAGGTCACCCTGAAGACCCT \\
\hline 31341 & CTCGCGGCCGCTTATGGTGCTGGTGTGGACACTGC \\
\hline 33589 & ATAGAATTCATGACCATCAGCCAGCAA \\
\hline 33590 & CTCGCGGCCGCTCAGTCTGCTGATTCCGCCT \\
\hline 1596 & TCGATGATGAAGATACCCCACC \\
\hline 1681 & AGAAATTGAGATGGTGCACGAT \\
\hline 28506 & TTCCAATGGGAGGACGACCAACCCAAACGATTCACTACAGAGCATGGGTTCTGA \\
\hline 28507 & TCAGAACCCATGCTCTGTAGTGAATCGTTTGGGTTGGTCGTCCTCCCATTGGAA \\
\hline 28504 & GCTTTTACCCGACAAGCACTGAACCTACGAGACAATACTGTCAGAGTTCAAGAC \\
\hline 28505 & GTCTTGAACTCTGACAGTATTGTCTCGTAGGTTCAGTGCTTGTCGGGTAAAAGC \\
\hline LacZ fwd & ATTCCAGCTGAGCGCCGGTCGC \\
\hline LacZ rev & GCGAGCTCAGACCATAACTTCGTATA \\
\hline $\begin{array}{l}\text { WT F7 } \\
\text { fwd }\end{array}$ & GGGCTGTATGAAGGAAGTGCTATT \\
\hline WT F7 rev & СCCTGAGAGTGAAGGGTGCTGTTC \\
\hline
\end{tabular}




\section{List of abbreviations}

${ }^{\circ} \mathrm{C}$

AAA-ATPase

AD

APP

APS

ATP

ATP13A2

ATPas

$\beta$-Gal

bp

BME

BSA

Cdk6

cDNA

cIAP1

cm

CNS

Co-IP

CP

CT

Cy

DAB

DAPI

DIV

DMEM

DmPI31

DNA
Degrees Celsius

ATPases Associated with diverse cellular Activities

Activator domain

Amyloid precursor protein

Ammonium persulfate

Adenosine triphosphate

ATPase type 13A2

Adenylpyrophosphatase

$\beta$-Galactosidase

Basepairs

Basal Medium Eagle

Bovine serum albumin

Cell division protein kinase 6

complementary DNA

Cellular inhibitor of apoptosis 1

Centimeter

Central nervous system

Co-Immunoprecipitation

Core particle

C-terminus

Cyanine

3-3'-diaminobenzidine

4'6-diamidino-2-phenylindole

Days in vitro

Dulbecco's modified Eagle's medium

Drosophila melanogaster proteasome inhibitor 31

Deoxyribonucleic acid 


$\begin{array}{ll}\text { DNA-BD } & \text { DNA-binding domain } \\ \text { dNTP } & \text { Deoxyribonucleotide triphosphate } \\ \text { DTT } & \text { Dithiothreitol } \\ \text { e.g. } & \text { exempli gratia } \\ \text { E1 } & \text { Ubiquitin-activating enzyme } \\ \text { E2 } & \text { Ubiquitin-conjugating enzyme } \\ \text { E3 } & \text { Ubiquitin ligase enzyme } \\ \text { ECL } & \text { Enhanced chemiluminescence } \\ \text { EDTA } & \text { Ethylenediaminetetraacetic acid } \\ \text { EGTA } & \text { Ethylene glycol tetraacetic acid } \\ \text { EIF4G1 } & \text { Eukaryotic translation initiation factor 4 gamma, 1 } \\ \text { En2-SA } & \text { Mouse engrailed 2 gene splice acceptor } \\ \text { ES } & \text { Embryonic stem } \\ \text { FBP } & \text { F-box protein } \\ \text { FBXL } & \text { F-box protein with leucine-rich repeats } \\ \text { FBXO } & \text { F-box protein with other domain motifs } \\ \text { FBXW } & \text { F-box protein with WD40 domains } \\ \text { FCS } & \text { Fetal calf serum } \\ \text { FLP } & \text { Flippase } \\ \text { FP } & \text { FBOX7-PI31 interaction domain } \\ \text { FRT } & \text { FLP-recognition target } \\ \text { G } & \text { Glycine } \\ \text { g } & \text { Gram } \\ \text { Gal4 } & \text { Galactose-responsive transcription factor 4 } \\ \text { Gal4-AD } & \text { Gal4 DNA-activation domain } \\ \text { Gal4-BD } & \text { Gal4 DNA-binding domain } \\ \text { GAPDH } & \text { Glyceraldehyde 3-phosphate dehydrogenase } \\ \text { GFAP } & \text { Glial fibrillary acidic protein } \\ \text { GFP } & \text { Green fluorescent protein } \\ \text { GST } & \text { Glutathione S-transferase } \\ \text { HBSS } & \text { Hank's Balanced Salt Soulution } \\ \text { HECT } & \text { Homologous to E6-AP carboxy terminal } \\ \text { HEK293T } & \text { HEPES }\end{array}$




\begin{tabular}{|c|c|}
\hline HRP & Horseradish peroxidase \\
\hline HS & Horse serum \\
\hline HtrA2 & HtrA serine peptidase 2 \\
\hline HURP & Hepatoma up-regulated protein \\
\hline I & Isoleucine \\
\hline IB & Immunoblot \\
\hline Iba1 & Ionized calcium-binding adapter molecule 1 \\
\hline ICC & Immunocytochemistry \\
\hline IgG & Immunoglobulin G \\
\hline IHC & Immunohistochemistry \\
\hline IP & Immunoprecipitation \\
\hline IPTG & Isopropyl $\beta-D-1$-thiohalactopyranoside \\
\hline IRES & Internal ribosome entry site \\
\hline K & Lysine \\
\hline Kan & Kanamycin \\
\hline kb & Kilobase \\
\hline kDa & Kilodalton \\
\hline $\mathbf{L}$ & Liter \\
\hline LC1, 2 & Light chain 1, 2 \\
\hline Leu & Leucine \\
\hline LRRK2 & Leucine-rich repeat kinase 2 \\
\hline LSAB2 & Labelled Streptavidin-Biotin2 System \\
\hline M & Methionine \\
\hline mA & Milliampere \\
\hline MAGD & Mitochondrial aggregation and genome destruction \\
\hline MAP1 & Microtubule-associated protein 1 \\
\hline Mda & Megadalton \\
\hline mg & Milligram \\
\hline ml & Milliliter \\
\hline $\mathrm{mm}^{2}$ & Square millimeter \\
\hline mRNA & Messenger RNA \\
\hline$\mu g$ & Microgram \\
\hline$\mu \mathbf{l}$ & Microliter \\
\hline$\mu \mathrm{M}$ & Micromolar \\
\hline
\end{tabular}




\begin{tabular}{|c|c|}
\hline$\mu \mathrm{m}$ & Micrometer \\
\hline mM & Milimolar \\
\hline mm & milimeter \\
\hline MPTP & 1-methyl-4-phenyl-1,2,3,6-tetrahydropyridine \\
\hline ms & millisecond \\
\hline NEM & $\mathrm{N}$-ethylmaleimide \\
\hline ng & Nanogram \\
\hline ns & non-significant \\
\hline NT & N-terminus \\
\hline OD & Optical density \\
\hline$P(n)$ & Postnatal Day (n) \\
\hline pA & Polyadenylation \\
\hline PBS & Phosphate-buffered saline \\
\hline PBST & Phosphate-buffered saline with Triton-X \\
\hline PCR & Polymerase chain reaction \\
\hline PD & Parkinson's disease \\
\hline PFA & Paraformaldehyde \\
\hline PMSF & Phenylmethanesulfonylfluoride \\
\hline PPS & Parkinsonian-pyramidal syndrome \\
\hline PRR & Proline-rich region \\
\hline PSG & Penicillin/Streptomycin with L-Glutamine \\
\hline $\mathrm{PI} 31$ & Proteasomal inhibitor 31 \\
\hline PSMA2 & Proteasomal subunit alpha 2 \\
\hline PSMB5 & Proteasomal subunit beta 5 \\
\hline PINK1 & PTEN Induced Putative Kinase 1 \\
\hline PTEN & Phosphatase and tensin homolog \\
\hline $\mathbf{R}$ & Arginine \\
\hline rb & Rabbit \\
\hline Rbx1 & RING-box protein 1 \\
\hline RING & Really Interesting New Gene \\
\hline RIPA & Radioimmunoprecipitation assay buffer \\
\hline RNA & Ribonucleic acid \\
\hline RNAi & RNA interference \\
\hline rpm & Revolutions per minute \\
\hline
\end{tabular}




$\begin{array}{ll}\text { Rpt6 } & \text { RP triphosphatase } \\ \text { RT } & \text { Room temperature } \\ \text { s.e.m. } & \text { Standard error of the mean } \\ \text { SCF } & \text { Skp, Cullin, F-box-containing complex } \\ \text { SDS } & \text { Sodium dodecyl sulfate } \\ \text { SDS-PAGE } & \text { SDS-Polyacrylamide gel electrophoresis } \\ \text { shRNA } & \text { Short hairpin RNA } \\ \text { SKP1 } & \text { S-phase kinase-associated protein 1 } \\ \text { SNCA } & \text { Synuclein, alpha (non A4 component of amyloid } \\ & \text { precursor) } \\ \text { SNP } & \text { Single nucleotide polymorphism } \\ \text { SP1 } & \text { Specificity protein 1 } \\ \text { T } & \text { Threonine } \\ \text { TAE } & \text { Tris base, Acetic acid and EDTA } \\ \text { TEMED } & \text { Tetramethylethylenediamine } \\ \text { TNF } & \text { Tumor necrosis factor } \\ \text { TRAF2 } & \text { TNF receptor-associated factor 2 } \\ \text { TUNEL } & \text { TdT-mediated dUTP Nick-End Labeling } \\ \text { TdT } & \text { Terminal deoxynucleotidyl transferase } \\ \text { Trp } & \text { Tryptophan } \\ \text { Ub } & \text { Ubiquitin } \\ \text { UbI } & \text { Ubiquitin-like } \\ \text { UCH-L1 } & \text { Ubiquitin carboxyl-terminal esterase L1 } \\ \text { UIM } & \text { Ubiquitin-interacting motif } \\ \text { UPS } & \text { Ubiquitin proteasome system } \\ \text { UV } & \text { Ultra violet } \\ \text { V } & \text { Volts } \\ \text { WB } & \text { Western blot } \\ \text { WT } & \text { Wildtype } \\ \text { X } & \text { Termination } \\ & \end{array}$




\section{Affidavit}

I hereby declare that this Ph.D. thesis entitled "The role of the E3 ubiquitin ligase FBX07-SCF in early-onset Parkinson's disease" has been written independently with no other sources that quoted.

David Brockelt

September, 2015

Göttingen, Germany 


\section{Curriculum Vitae}

\section{Personal information}

$\begin{array}{ll}\text { Date of birth } & 02.02 .1987 \\ \text { Place of birth } & \text { Berlin, Germany } \\ \text { Nationality } & \text { German } \\ \text { Address } & \text { Max Planck Institute for Experimental Medicine } \\ & \text { Hermann-Rein-Str. 3 } \\ & 37075, \text { Göttingen } \\ & \text { Germany } \\ \text { Phone number } & \text { (+49) 5513899564 } \\ \text { Email } & \text { Brockelt@em.mpg.de }\end{array}$

\section{Education}

October 2011- Current

Ph.D. student at the M.Sc./Ph.D. IMPRS Neuroscience program Göttingen, Germany. Project title: "The role of the E3 ubiquitin ligase FBXO7-SCF in early-onset Parkinson's disease". Supervisor Dr. Judith Stegmüller

\section{September 2010- August 2011}

M.Sc. student at the M.Sc./Ph.D. IMPRS Neuroscience program Göttingen, Germany. M.Sc. thesis waived, direct admission to Ph.D.

\section{October 2007- August 2010}

B.Sc. in Biology at the Georg-August-Universität Göttingen. Project title: "Functional characterization of the interaction of Disrupted-in-Schizophrenia-1 (DISC1) and F-box protein 31 (FBXO31)". Supervisor: Dr. Judith Stegmüller

\section{Publication}

Shinoda, Y., Ahmed, S., Ramachandran, B., Bharat, V., Brockelt, D., Altas, B., \& Dean, C. (2014). BDNF enhances spontaneous and activity-dependent neurotransmitter release at excitatory terminals but not at inhibitory terminals in hippocampal neurons. Frontiers in synaptic neuroscience, 6. 


\section{Conferences}

Neurizons 2013: $\quad$ Solving the Brain Puzzle- organizer, poster.

EMBO Conference 2013: $\quad$ Ubiquitin \& Ubiquitin-like Proteins: From Structure to Functionposter

Teaching experience

December 2013

May - June 2013

Jan - Mar 2013

December 2012
Tutor for methods course on "Neurobiology I" for the M.Sc. program "Developmental, Neural, and Behavioral Biology" Supervisor of laboration student Sabitha Joseph Supervisor of laboration student Guergana Dontcheva Tutor for methods course on "Neurobiology I" for the M.Sc. program "Developmental, Neural, and Behavioral Biology" 